\title{
A distribuição log-logística exponenciada geométrica: dupla ativação
}

\section{Natalie Verónika Rondinel Mendoza}

\section{Piracicaba}


Natalie Verónika Rondinel Mendoza

Licenciada em Matemática

\section{A distribuição log-logística exponenciada \\ geométrica: dupla ativação}

versão revisada de acordo com a resolução CoPGr 6018 de 2011

Orientador:

Prof. Dr. EDWIN MOISES MARCOS ORTEGA

Dissertação apresentada para obtenção do título de Mestre em Ciência. Área de concentração: Estatística e Experimentação Agronômica

Piracicaba

2012 


\section{Dados Internaclonais de Catalogação na Publicação DIVISÄO DE BIBLIOTECA - ESALQ/USP}

\section{Mendoza, Natalie Verónika Rondinel}

A distribuiçäo log-logística exponenciada geométrica: dupla ativação / Natalie Verónika Rondinel Mendoza.- - versão revisada de acordo com a resoluçăo $\mathrm{CoPGr}$ 6018 de 2011. . - Piracicaba, 2012

$100 \mathrm{p}$ : it.

Dissertação (Mestrado) - - Escola Superior de Agricultura "Luiz de Queiroz", 2012.

1. Análise de sobrevivência 2. Dados censurados 3. Distribuiçảo geométrica 4. Distribuição log-logística 5 . Função de taxa de fatha 6 . Modelos de regressão 7. Verossimilhança I. Titulo

$\operatorname{CDD} 519.546$

M539d 


\section{Dedicatória}

Com amor e carinho agradeço a Deus, meu Pai do ceú por ter colocado muitas provas neste caminho da vida (e que ainda irá colocar), porque eu sei que com dedicação, fé e paciência fará que eu nunca perca as forças .

Com todo meu amor a meus pais,

Sócrates Rondinel e Fortunata Mendoza, a minha irmãzinha, Katy Rondinel Mendoza, que sempre me apoiam com amor incondicional e alegram minha vida, mesmo estando a kilômetros de distância.

A meu irmão Juan Carlos Rondinel Mendoza (in memoriam), quem foi meu anjo da guarda durante toda esta jornada, dedico este trabalho, saudades eternas..... 


\section{AGRADECIMENTOS}

A meus pais: Sócrates Rondinel e Fortunata Mendoza, a quem eu amo profundamente, por estarem ao meu lado e por ter dado para mim o tesouro mais valioso que pode-se dar a uma filha, AMOR. Quem sem minimizar esforço nenhum, têm sacrificado grande parte da sua vida, para me formar e educar, e converter-me em pessoa de sucesso, a quem eu nunca poderei pagar com as riquezas mais grandes do mundo. Sinceramente... muito agradecida!.

A minha irmã Katy Rondinel, por todo o seu apoio, ajuda e pensamento positivo. Sempre me fazendo olhar para frente, nunca me deixando abalar com adversidades e NUNCA desistir. Amo a você muito.

Aos meus amigos: Rosario, Gina, Diana, Priscila, Carlos, Javier, Jhoana, Juan Carlos e Pedro, pelos momentos de alegria, descontração e discussões sobre valores que sempre fizeram abrir meus olhos para outros pontos de vista e por estarem ao meu lado nos momentos de aperto sempre oferecendo seu apoio, obrigada pela grande amizade que me proporcionaram.

Aos meus amigos e colegas de pós-graduação: Elizabeth, Fabiane, Kuang, Ana Julia, João, Patricia, Luiz Ricardo, Gabriel, Daniel, Italo, Simone, Renata, Marina e Maria Cristina, por estarem ao meu lado.

Em especial à Senhorita Luciane Brajão por ter acreditado em mim e dar-me toda a ajuda nos momentos cruciais e pela grande amizade.

Agradeço em especial ao meu orientador Professor Dr. Edwin Moises Marcos Ortega, pela orientação, ajuda e pelas conversas para o desenvolvimento deste trabalho.

Aos Professores do programa de pós graduação em Estatística e Experimentação Agronômica: Clarice Garcia Borges Demétrio, Carlos Tadeu dos Santos Dias, Roseli Aparecida, Silvio Sandoval Zocchi, Sônia Maria De Stefano Piedade, César Gonçalves De Lima, Gabriel Sarrés e Taciana Villela Savian pelos conselhos e ajuda nos momentos de dúvidas.

A Senhorita Secretária Solange e funcionários do programa de pós-graduação: Eduardo, Jorge e Rosni pelos auxílios permanentes.

Agradeço a todas as pessoas que de uma forma direta ou indireta contribuíram 
para o meu crescimento profissional.

Por fim a todos que sempre acreditaram em mim.

OBRIGADA!!!!!! 


\section{SUMÁRIO}

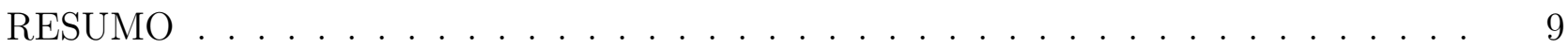

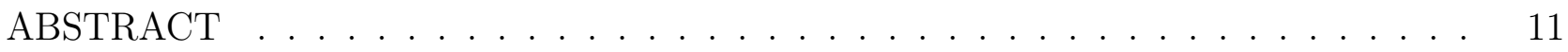

LISTA DE FIGURAS . . . . . . . . . . . . . . . . . . . . . . . . 13

LISTA DE TABELAS . . . . . . . . . . . . . . . . . . . . . . . 15

1 INTRODUÇÃO . . . . . . . . . . . . . . . . . . . . . . . . 17

2 DESENVOLVIMENTO . . . . . . . . . . . . . . . . . . . . . 19

2.1 Conceitos básicos de análise de sobrevivência . . . . . . . . . . . . . . . . . 19

2.2 Classe de distribuições exponenciadas . . . . . . . . . . . . . . . . . . 22

3 DISTRIBUIÇÃO LOG-LOGÍSTICA EXPONENCIADA GEOMÉTRICA . . . . . . . 25

3.1 Distribuição log-logística . . . . . . . . . . . . . . . . . . . . . . 25

3.2 Distribuição log-logística exponenciada . . . . . . . . . . . . . . . . . . . . . 29

3.3 Distribuição log-logística exponenciada geométrica com dupla ativação . . . . . . 33

3.3.1 Distribuição log-logística exponenciada geométrica tipo I ～. . . . . . . . . . . 33

3.3.2 Distribuição log-logística exponenciada geométrica tipo II . . . . . . . . . . . . . . 36

3.4 Propriedades da log-logística exponenciada geométrica . . . . . . . . . . . . . . 40

3.4.1 Expansão da função densidade da distribuição log-logística exponenciada geométrica 40

3.4 .2 Momentos . . . . . . . . . . . . . . . . . . . . . . . 45

3.4.2.1 Assimetria . . . . . . . . . . . . . . . . . . . 47

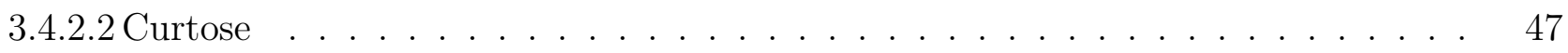

3.4.3 Função Geradora de Momentos . . . . . . . . . . . . . . . . . . . . . . . . 48

3.4 .4 Desvios médios . . . . . . . . . . . . . . . . . . . . . . . . 51

3.4.5 Curvas de Bonferroni e Lorenz . . . . . . . . . . . . . . . . . . . 54

3.5 Estimação por Máxima Verossimilhança . . . . . . . . . . . . . . . . 55

3.5.1 Testes de hipóteses . . . . . . . . . . . . . . . . . . . . . . 58

4 DISTRIBUIÇÃO LOGÍSTICA EXPONENCIADA GEOMÉTRICA . . . . . . . . . 59

4.1 Distribuição logística exponenciada . . . . . . . . . . . . . . . . . . 59

4.2 Distribuição logística exponenciada geométrica com dupla ativação . . . . 59

4.2.1 Expansão da função densidade da distribuição logística exponenciada geométrica . 61

4.3 Momentos . . . . . . . . . . . . . . . . . . . . . 64

4.4 Função Geradora de Momentos . . . . . . . . . . . . . . . . . . . . 64

4.5 Modelos de Regressão . . . . . . . . . . . . . . . . . . . . . . . . . . 65

4.5.1 Modelo de locação e escala . . . . . . . . . . . . . . . . . . . . 65 
4.5.2 Modelo de regressão logístico exponenciada geométrico . . . . . . . . . . . 66 4.6 Estimação dos parâmetros . . . . . . . . . . . . . . . . . . . 66 5 APLICAÇÕES . . . . . . . . . . . . . . . . . . . . . . 71

5.1 Aplicação 1: Dados de contaminação do ambiente . . . . . . . . . . . . . 71

5.2 Aplicação 2: Dados de nível de voltagem . . . . . . . . . . . . . . . . 73 6 CONSIDERAÇÕES FINAIS . . . . . . . . . . . . . . . . . . 77

6.1 Conclusões . . . . . . . . . . . . . . . . . . . . . . 77

6.2 Trabalhos futuros . . . . . . . . . . . . . . . . . 77

REFERENCIAS . . . . . . . . . . . . . . . . . . . . . 79

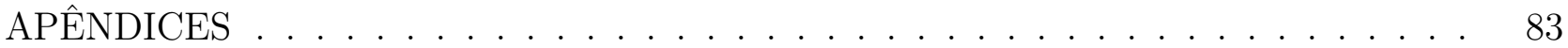




\section{RESUMO}

\section{A distribuição log-logística exponenciada geométrica: dupla ativação}

Neste trabalho é proposta uma nova distribuição de quatro parâmetros denominada distribuição log-logística exponenciada geométrica, baseada em um mecanismo de dupla ativação para modelar dados de tempo de vida. Para esta nova distribuição, foi realizado um estudo da função de densidade de probabilidade, da função de distribuição acumulada, da função de sobrevivência e da função de taxa de falha, a qual apresenta formas que podem modelar dados de tempo de vida, tais como: forma crescente, decrescente, unimodal, bimodal e forma de U. Obteve-se expansões da função de densidade, expressões para os momentos de probabilidade ponderada, função geradora de momentos, desvios médios e as curvas de Bonferroni e de Lorenz. Considerando dados censurados, foi utilizado o método de máxima verossimilhança para estimação dos parâmetros. Analogamente também é proposto um modelo de regressão baseado no logaritmo da distribuição log-logística exponenciada geométrica com dupla ativação, que é uma extensão dos modelos de regressão logística exponenciada e logística. Este modelo pode ser usado na análise de dados reais, por fornecer um melhor ajuste que os modelos de regressão particulares, logística exponenciada e logística. Finalmente, são apresentados duas aplicações para ilustrar a utilização da nova distribuição.

Palavras-chave: Dados Censurados; Distribuição geométrica; Distribuição log-logística; Distribuição log-logística exponenciada; Função de taxa de falha; Máxima verossimilhança; Modelo de regressão 


\section{ABSTRACT \\ The exponentiated log-logistic geometric distribution: dual activation}

In this work, we propose a new distribution with four parameters the so called exponentiated log-logistic geometric distribution based on a double mechanism of activation for modeling lifetime data. For this new distribution, we study the density function, cumulative distribution, survival function and the failure rate function which allows major harzad rates: increasing, decreasing, bathtub, unimodal and bimodal failure rates. We also obtain the density function expansions and the expressions for the probability-weighted moments, moment generating function, mean deviation and Bonferroni and Lorenz curves. Considering censored data, we use the maximum likelihood method for estimating the parameters. Similarly, we also propose the regression model based on the logarithm of the exponentiated log-logistic geometric distribution with double activation, which is an extension of the exponential logistic and logistic regression models. This new model could be widely used in the analysis of real data to provide a better fit than exponetial logistic and logistic regression models. Finally, two applications are presented to illustrate the application of the new distribution.

Keywords: Censored data; Exponentiated log-logistic distribution; Function of failure rate; Geometric distribution; Log-logistic distribution; Maximum likelihood;

Regression model 


\section{LISTA DE FIGURAS}

Figura 1 - Gráfico de diferentes funções de taxa de falha . . . . . . . . . . . . . . .

Figura 2 - Gráficos da função densidade (a), de sobrevivência (b) e de taxa de falha (c) da distribuição log-logística, para alguns valores do parâmetro $\beta$ fixando $\alpha=2527$

Figura 3 - Gráficos da fdp da distribuição LLE, para alguns valores dos parâmetros. (a) Para diferentes valores de $\alpha$ e $a$, fixando $\beta=3,5$. (b) Para diferentes valores de $\beta$ e $a$, fixando $\alpha=2,5$. (c) Para diferentes valores de $a$, fixando $\alpha=0,8$

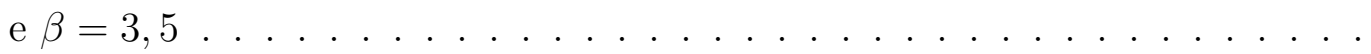

Figura 4 - Gráficos da função de sobrevivência da distribuição LLE, para alguns valores dos parâmetros. (a) Para diferentes valores de $\alpha$ e $a$, fixando $\beta=3,5$. (b) Para diferentes valores de $\beta$ e $a$, fixando $\alpha=2,5$. (c) Para diferentes valores de $a$, fixando $\alpha=0,8$ e $\beta=3,5 \ldots \ldots \ldots$

Figura 5 - Gráficos da função de taxa de falha da distribuição LLE, para alguns valores dos parâmetros. (a) Para diferentes valores de $\alpha$ e $a$, fixando $\beta=3,5$. (b) Para diferentes valores de $\beta$ e $a$, fixando $\alpha=2,5$. (c) Para diferentes valores de $a$, fixando $\alpha=1,7$ e $\beta=2,9 \ldots \ldots \ldots$

Figura 6 - Gráficos da fdp da distribuição LLEGI, para alguns valores dos parâmetros. (a) Para diferentes valores de $a, p$ e $\alpha$, fixando $\beta=4,5$. (b) Para diferentes valores de $a, p$ e $\beta$, fixando $\alpha=1,5$. (c) Para diferentes valores de $a$ e $p$, fixando $\alpha=0,5$ e $\beta=3,5 \ldots \ldots \ldots \ldots$

Figura 7 - Gráficos da função de taxa de falha da distribuição LLEGI, para alguns valores dos parâmetros. (a) Para diferentes valores de $a, p$ e $\beta$, fixando $\alpha=1,5$. (b) Para diferentes valores de $a, p$ e $\alpha$, fixando $\beta=4,5$. (c) Para diferentes valores de $a, p$ e $\beta$, fixando $\alpha=1,5 \ldots \ldots \ldots$

Figura 8 - Gráficos da fdp da distribuição LLEGII, para alguns valores dos parâmetros. (a) Para diferentes valores de $a, p$ e $\alpha$, fixando $\beta=4,5$. (b) Para diferentes valores de $a, p$ e $\beta$, fixando $\alpha=1,5$. (c) Para diferentes valores de $a$ e $p$, fixando $\alpha=0,5$ e $\beta=3,5 \ldots \ldots \ldots \ldots$

Figura 9 - Gráficos da taxa de falha da distribuição LLEGII, para alguns valores dos parâmetros. (a) Para diferentes valores de $a, p$ e $\alpha$, fixando $\beta=2,5$. (b) Para diferentes valores de $a, p$ e $\beta$, fixando $\alpha=1,5$. (c) Para diferentes valores de a e $p$, fixando $\alpha=1,5$ e $\beta=3,5 \ldots \ldots \ldots$ 
Figura 10 - Assimetria da distribuição LLEGI para alguns valores dos parâmetros. (a) Como função de $p$ para alguns valores de $a$ fixando $\alpha=1,5$ e $\beta=3,5$. (b) Como função de $a$ para alguns valores de $p$ fixando $\alpha=1,5$ e $\beta=3,5$. . .

Figura 11 - Assimetria da distribuição LLEGII para alguns valores dos parâmetros. (a) Como função de $p$ para alguns valores de $a$ com $\alpha=1,5$ e $\beta=3,5$. (b) Como função de $a$ para alguns valores de $p$ fixando $\alpha=1,5$ e $\beta=3,0$. . .

Figura 12 - Curtose da distribuição LLEGI para alguns valores dos parâmetros. (a) Como função de $p$ para alguns valores de $a$ fixando $\alpha=1,5$ e $\beta=4,5$. (b) Como função de $a$ para alguns valores de $p \operatorname{com} \alpha=1,0$ e $\beta=5,5 \ldots \ldots$. . .

Figura 13 - Curtose da distribuição LLEGII para alguns valores dos parâmetros. (a) Como função de $p$ para alguns valores de $a$ com $\alpha=1,5$ e $\beta=3,0$. (b) Como função de $a$ para alguns valores de $p$ considerando $\alpha=1,5$ e $\beta=3,5$ 50

Figura 14 - Gráficos da fdp da distribuição LEGI, para alguns valores dos parâmetros. (a) Para diferentes valores de $a$ e $p$, com $\mu=0$ e $\sigma=1$. (b) Para diferentes valores de $a, p$ e $\mu$, com $\sigma=1$. (c) Para diferentes valores de $a, p, \mu$ e $\sigma$.

Figura 15 - Gráficos da fdp da distribuição LEGI, para alguns valores dos parâmetros. (a) Para diferentes valores de $a$ e $p$, com $\mu=0$ e $\sigma=1$. (b) Para diferentes valores de $a, p$ e $\mu$, com $\sigma=1$. (c) Para diferentes valores de $a, p, \sigma$ e $\mu=-162$

Figura 16 - (a) Densidade LLEGII ajustada para os dados de Contaminação. (b) Função de distribuição acumulada estimado da distribuição LLEGII para os dados de contaminação . . . . . . . . . . . . . . . . . . . .

Figura 17 - Curvas da função de sobrevivência estimada para o modelo LEGII para os dados de níveis de voltagem f . . . . . . . . . . . . . . . 76 


\section{LISTA DE TABELAS}

Tabela 1 - Estatísticas descritivas . . . . . . . . . . . . .

Tabela 2 - Estimativas de máxima verossimilhança dos parâmetros do modelo para dados de contaminação e o critério de informação . . . . . . . . . . . . . . . 73

Tabela 3 - Teste da razão de verossimilhança . . . . . . . . . . . . . . . 73

Tabela 4 - Estimativas de máxima verossimilhança dos parâmetros dos modelos de regressão ajustados aos dados de níveis de voltagem, seus correspondentes EPs (em parênteses), p-valor em [.] e as estatísticas AIC, CAIC e BIC . . . . 75

Tabela 5 - Teste da razão de verossimilhança . . . . . . . . . . . . . . . 76 


\section{INTRODUÇÃO}

Em aplicações na área de análise de sobrevivência, é frequente a ocorrência de função de taxa de falha em forma de U ou unimodal; isto é, funções não-monótonas. Os modelos comumente usados para dados de sobrevivência são a distribuição Weibull, com função de taxa de falha monótona e a distribuição log-logística com função de taxa de falha decrescente ou unimodal. Por outro lado, nos últimos anos tem crescido muito a generalização e, ou, modificação de algumas distribuições utilizadas em análise de sobrevivência; assim destaca-se, a distribuição Weibull exponenciada (WE) proposta por Mudholkar et al. (1995) e Mudholkar et al. (1996). A distribuição WE é uma generalização da distribuição Weibull, e possui várias propriedades, dentre elas, a sua função de taxa de falha pode modelar diferentes formas (constante, crescente, decrescente, unimodal e forma de U). Adamidis e Loukas (1998) introduziram a distribuição exponencial geométrica (EG), para modelar dados de tempo de vida com função taxa de falha decrescente. Kus (2007) estudou uma nova distribuição com função taxa de falha decrescente. Essa distribuição é obtida por meio da mistura das distribuições exponencial e Poisson truncada. Gupta e Kundu (1999, 2001, 2007) forneceram um tratamento matemático compreensível da chamada distribuição exponencial generalizada. Carrasco et al. (2008a) propuseram a distribuição Weibull modificada generalizada com aplicações na análise de sobrevivência. Seguindo a mesma idéia da distribuição exponencial geométrica, Silva et al. (2010) definiram a distribuição exponencial geométrica generalizada e demonstraram que a função taxa de falha pode ser crescente, decrescente ou unimodal. Uma extensão da distribuição EG é a distribuição Weibull geométrica, proposta por Barreto-Souza et al. (2011) o qual modela uma função taxa de falha monótona ou unimodal.

A distribuição log-logística proposta por Tadikamalla e Jhonson (1982) tem-se apresentado como alternativa às distribuições Weibull e log-normal para modelar dados de tempo de vida e caracteriza-se por modelar funções de taxa de falha decrescente e unimodal para alguns valores dos parâmetros da distribuição. Além disso, esta distribuição possui a mesma relação que a distribuição log-normal tem com a normal (Ahmad et al.(1988)), ou seja, se $T$ segue uma distribuição log-logística, então $Y=\log (T)$ segue uma distribuição logística.

Algumas aplicações da distribuição log-logística são discutidas em economia, por exemplo, para modelar a distribuição de riqueza e renda (Kleiber e Kotz, 2003) e na área de hidrologia é utilizada para escoamento fluvial. Collet (2003) sugeriu a distribuição log-logística para modelar o tempo na sequência de um transplante de coração. 
Portanto, o objetivo deste trabalho, é propor a distribuição log-logística exponenciada geométrica baseado em uma dupla ativação, para modelar dados de tempo de vida, cuja distribuição é uma extensão da distribuição log-logística exponenciada proposta por Rosaiah et al. (2006). As novas generalizações das distribuições considerando a dupla ativação, serão denominadas de distribuições: log-logística exponenciada geométrica tipo I (LLEGI), log-logística exponenciada geométrica tipo II (LLEGII), logística exponenciada geométrica tipo I (LEGI) e logística exponenciada geométrica tipo II (LEGII). Apresenta-se propriedades destas novas distribuições, tais como expansões da função densidade de probabilidade em série ponderada de potência, os momentos e a função geradora de momentos, algumas medidas como a função do desvio médio da média e da mediana, as curvas de Bonferroni, as curvas de Lorenz, e as estimações por máxima verossimilhança. Além disso, será proposto o modelo de regressão baseado no logaritmo da variável aleatória que segue uma distribuição LLEGI ou LLEGII, finalmente, duas aplicações são apresentadas ilustrando o melhor ajuste das novas distribuições em relação às distribuições originais. 


\section{DESENVOLVIMENTO}

\subsection{Conceitos básicos de análise de sobrevivência}

Na atualidade, apresentam-se com muita freqüência eventos ou experimentos em estudo que envolvem um tempo de vida ou de sobrevivência; isto é, observa-se o tempo a partir do início do estudo até a ocorrência de um evento de interesse.

Portanto, o conjunto de técnicas estatísticas adequadas para estudar e analisar este tipo de dados é conhecido como análise de sobrevivência, em que a variável resposta é geralmente, o tempo até a ocorrência de um evento de interesse. Este tempo é denominado tempo de falha, evento que ocorre, quando o indivíduo sob estudo, apresenta a característica de interés para terminar o estudo (por exemplo, podendo ser o tempo até a morte do paciente, bem como até a cura ou recidiva de uma doença, etc.) e a sua relação com outras possíveis variáveis explanatórias. Os indivíduos sob estudo podem ser animais, seres humanos, plantas, equipamentos, etc.

A principal característica de dados de sobrevivência é a presença de censura, que pode ser interpretada como, uma observação parcial da resposta ou tempo de falha, devido à perda ou retirada de um indivíduo sob estudo; por exemplo, a situação em que por alguma razão o acompanhamento do paciente foi interrompido, seja porque o paciente mudou de cidade ou o estudo foi concluído ou o paciente morreu de uma causa diferente da estudada, isto significa que toda informação referente à resposta se resume ao conhecimento de que o tempo de falha é superior àquele observado (Colosimo e Giolo, 2006). Porém a censura pode ser classificada como censura à direita, quando a falha ocorre sempre à direita do tempo registrado, a censura à esquerda, quando o tempo registrado é maior que o tempo de falha. A censura intervalar, em que não se sabe o tempo exato de falha, sabe-se apenas que ele ocorreu em um certo intervalo de tempo.

As censuras à direita podem ainda ser caracterizadas como censura do tipo I, que é aquela em que o estudo será terminado após um período pré-estabelecido de tempo. Censura do tipo II é aquela em que o estudo será terminado após ter ocorrido o evento de interesse em um número pré-estabelecido de indivíduos. Censura aleatória, o que mais ocorre na prática, este tipo de censura acontece, quando o indivíduo é retirado do estudo, sem ter ocorrido a falha, ou também, se o indivíduo morrer por alguma razão diferente da estudada. Neste trabalho, será adotado o mecanismo de censura aleatória à direita.

Seja $T$ uma variável aleatória não negativa que representa o tempo de falha de 
um indivíduo e seja $C$ outra variável aleatória independente de $T$, que representa o tempo de censura associado a este indivíduo. Assim, os dados observados são representados por $t=\min (T, C)$ e $\delta$ é o indicador de censura, expressa por

$$
\delta= \begin{cases}1, & \text { se } T \leqslant C \\ 0, & \text { se } T>C\end{cases}
$$

em que $\delta=0$ indica censura e $\delta=1$ indica falha.

A distribuição de probabilidade da variável aleatória $T$ pode ser especificada por meio da função densidade de probabilidade, função de sobrevivência ou função de taxa de falha, sendo as três formas equivalentes.

Seja $T$ uma variável aleatória não negativa contínua, a função densidade de probabilidade, $f(t)$, é uma função definida em $\mathbb{R}^{+}$, tal que

$$
f(t)=\lim _{\triangle t \rightarrow 0}\left(\frac{1}{\triangle t}\right) P(t \leqslant T \leqslant t+\triangle t),
$$

em que $\triangle t$ é um incremento temporal.

A função de sobrevivência, denotada por $S(t)$, é definida como a probabilidade do tempo de falha de uma observação não falhar até um certo tempo $t$, isto é

$$
S(t)=P(T \geqslant t)=1-F(t)=\int_{t}^{\infty} f(t) d t
$$

A taxa de falha num intervalo $[t, t+\triangle t]$ é definida como a probabilidade de que a falha ocorra nesse intervalo, dado que não ocorreu antes do tempo $t$, dividida pelo comprimento do intervalo de tempo.

Assumindo um intervalo de tempo pequeno, $\Delta t \rightarrow 0, h(t)$ representa a razão instantânea de falha ou morte no tempo $t$ de um indivíduo, dado que ele sobreviveu até o tempo $t$, ou seja, $h(t)$ é denominado função de taxa de falha ou de risco, e é definido como

$$
h(t)=\lim _{\triangle t \rightarrow 0} \frac{P(t \leqslant T<t+\Delta t \mid T \geqslant t)}{\triangle t}=\frac{f(t)}{S(t)},
$$

em que, $f(t)$ e $S(t)$ são as funções densidade de probabilidade e de sobrevivência, respectivamente.

A função de taxa de falha, $h(t)$, é muito útil para descrever a distribuição do tempo de vida de indivíduos em estudo, pois ela determina a forma em que a taxa instantânea 
de falha se altera ao longo do tempo, expressa por

$$
h(t)= \begin{cases}h_{1}(t), & t>t_{3} \\ h_{2}(t), & t_{2}<t<t_{3} \\ h_{3}(t), & t_{1}<t<t_{2}\end{cases}
$$

O gráfico da função de taxa de falha pode apresentar algumas formas básicas, conforme se mostra na Figura 1. A função $h_{1}(t)$, é crescente, o que indica que a taxa de falha da observação aumenta com o transcorrer do tempo, este comportamento mostra um efeito gradual de envelhecimento. A função $h_{2}(t)$, é constante, e indica que a taxa de falha não se altera com o passar do tempo. A função $h_{3}(t)$, é decrescente, mostra que a taxa de falha diminui à medida que o tempo passa. Portanto, as três formas de taxa de falha básicas se combinam para gerar a curva da banheira ou a forma de U.

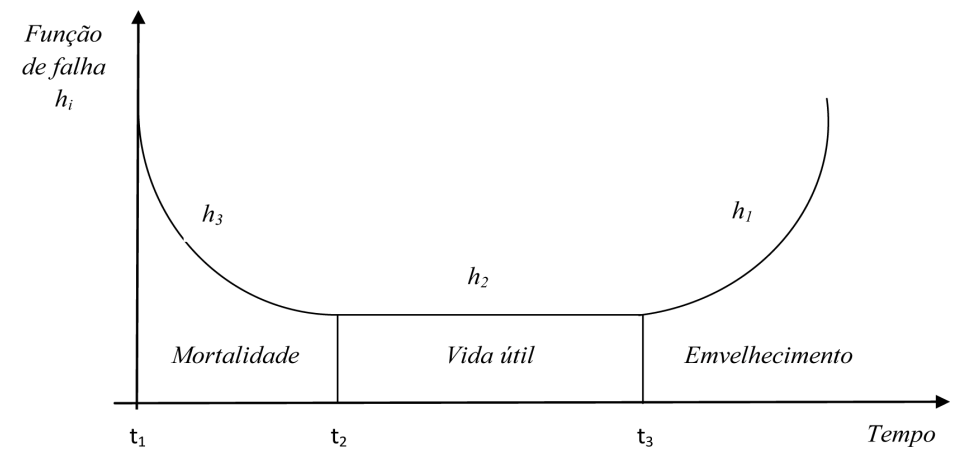

Figura 1 - Gráfico de diferentes funções de taxa de falha

A função de taxa de falha é mais informativa do que a função de sobrevivência, pois diferentes funções de sobrevivência podem ter formas semelhantes e funções de taxa de falha totalmente diferentes. Portanto, a modelagem da função de taxa de falha é um importante método para análise de dados de tempo, segundo Colosimo e Giolo (2006). 


\subsection{Classe de distribuições exponenciadas}

Há diferentes formas de modificar uma distribuição de probabilidade, em particular existem as classes de distribuições exponenciadas; isto é, considere-se $T$ uma variável aleatória positiva com função de distribuição acumulada (fda) conhecida $G(t)$, então a nova distribuição pode ser obtida exponenciando a fda base $G(t)$; expressa por

$$
F(t)=G(t)^{a}, \quad t>0,
$$

em que $a>0$ é um novo parâmetro que caracteriza a forma da nova distribuição; isto é, flexibiliza a distribuição base.

Consequentemente a função densidade de probabilidade (fdp) é dada por

$$
f(t)=a g(t) G(t)^{a-1}
$$

A função de sobrevivência e a função de taxa de falha são dados por

$$
S(t)=1-G(t)^{a} \quad \text { e } \quad h(t)=\frac{a g(t) G(t)^{a-1}}{1-G(t)^{a}}
$$

respectivamente.

Nos últimos anos, diversos trabalhos envolvendo esta classe de distribuições foram propostos, dentre os quais destaca-se a distribuição Weibull exponenciada (WE) proposta por Mudholkar et al. $(1995,1996)$ que é uma generalização da distribuição Weibull, esta distribuição possui várias propriedades, dentre elas, destaca-se que a função de taxa de falha pode modelar diferentes formas (constante, crescente, decrescente, unimodal e forma de U).

Mudholkar et al. $(1995,1996)$ analisaram e discutiram diferentes aplicações de dados de sobrevivência, esta distribuição também possui algumas distribuições conhecidas como casos particulares.

No entanto, Gupta e Kundu (1999, 2001) propuseram a distribuição exponencial generalizada também conhecida como distribuição exponencial exponenciada (EE), Gupta e Kundu (2001) observaram que esta distribuição possui algumas características interessantes que são similares àquelas das distribuições gama e Weibull. Dessa forma, este modelo pode ser usado como alternativa aos modelos gama e Weibull em diversas situações.

Segundo Gupta e Kundu (2001), uma das vantagens dessa distribuição é devida às estruturas simples de suas funções de distribuição e sobrevivência, a distribuição EE pode ser usada de forma eficaz na análise de dados de sobrevivência, particularmente na presença de observações censuradas ou dados correlacionados. Gupta e Kundu (2007), descreveram 
vários métodos para a obtenção das estimativas dos parâmetros, como o método de máxima verossimilhança, o método dos momentos, o método dos mínimos quadrados e os estimadores L-momentos.

A distribuição Rayleigh generalizada proposta por Kundu e Raqab (2005), pode ser utilizada em modelagem de dados de tempo de vida em geral. Nadarajah e Kotz (2006b), discutiram algumas distribuições exponenciadas e estendidas, assim desenvolveram as propriedades básicas de cada distribuição, entre estas distribuições tem-se, a distribuição gama exponenciada, Gumbel exponenciada e a distribuição Fréchet exponenciada. Estas distribuições são generalizações das distribuições gama padrão, Gumbel padrão e distribuição Fréchet padrão, respectivamente.

Mostraram Nadarajah e Kotz (2006b) que a função de taxa de falha da distribuição gama exponenciada, modela formas decrescente, unimodal e constante, assim como apresentaram a função geradora de momentos e os momentos dessa distribuição. Nadarajah (2006) apresentou um tratamento detalhado das propriedades dessa distribuição, apresentando as formas analíticas da fdp e da função taxa de falha, assim como também as propriedades básicas da distribuição Fréchet exponenciada.

Nesse mesmo enfoque, Rosaiah et al. (2006) propuseram a distribuição loglogística exponenciada, baseada na distribuição log-logística que foi proposta por Tadikamalha e Jhonson (1982), na qual Rosaiah et al. (2006) estudaram os tempos de vida de produtos que seguem a distribuição log-logística exponenciada, mas não foram discutidas as propriedades dessa distribuição. Neste trabalho apresenta-se algumas propriedades da distribuição loglogística exponenciada.

A distribuição Weibull modificada generalizada proposta por Carrasco et al. (2008a) apresenta função taxa de falha com formas crescente, decrescente, forma de U e unimodal, e possui como casos particulares a distribuição Weibull, Weibull exponenciada, exponencial exponenciada, entre outros.

Por outro lado, Cordeiro et al. (2011) propuseram a distribuição gama generalizada exponenciada, Alkasasbeh e Raqab (2009) estudaram a distribuição logística generalizada, definida por Balakrishnam e Leung (1998), na qual as estimativas dos parâmetros foram obtidas por meio do método de máxima verossimilhança. 


\section{DISTRIBUIÇÃO LOG-LOGÍSTICA EXPONENCIADA GEOMÉTRICA}

Nesta seção serão estudadas as distribuições log-logística, log-logística exponenciada, log-logística exponenciada geométrica com dupla ativação, serão apresentadas as principais características e propriedades dessas distribuições.

\subsection{Distribuição log-logística}

Em muitas situações práticas, a distribuição log-logística proposta por Tadikamalla e Johnson (1982) tem-se apresentado como alternativa às distribuições de Weibull e a log-normal, conforme discutem Colosimo e Giolo(2006). No entanto, em econômia onde é conhecida como a distribuição Fisk, modela a distribuição de riqueza e renda (Kleiber e Kotz, 2003) e na área de hidrologia é utilizada para escoamento fluvial (Ashkar e Mahdi, 2006).

Por outro lado, Collet (2003) sugeriu a distribuição log-logística para modelar o tempo na sequência de um transplante de coração.

Seja $Y$ uma variável aleatória com distribuição log-logística (LL), cuja função densidade de probabilidade, denotada por $g(y)$, é dada por

$$
g(y)=\frac{\beta}{\alpha}\left(\frac{y}{\alpha}\right)^{\beta-1}\left[1+\left(\frac{y}{\alpha}\right)^{\beta}\right]^{-2}, \quad y>0,
$$

em que $\alpha>0$ é o parâmetro de escala e $\beta>0$ é o parâmetro de forma.

Se $Y$ é uma variável aleatória positiva com distribuição LL com parâmetros $\alpha$ e $\beta$, então, denota-se que $Y \sim \operatorname{LL}(\alpha, \beta)$. As funções de distribuição acumulada denotada por $G(y)$, de sobrevivência $S(y)$ e de taxa de falha $h(y)$ são expressas respectivamente, por

$$
\begin{gathered}
G(y)=\left(\frac{y}{\alpha}\right)^{\beta}\left[1+\left(\frac{y}{\alpha}\right)^{\beta}\right]^{-1}=\left[1+\left(\frac{y}{\alpha}\right)^{-\beta}\right]^{-1}, \\
S(y)=\left[1+\left(\frac{y}{\alpha}\right)^{\beta}\right]^{-1} \mathrm{e} \\
h(y)=\frac{\beta}{\alpha}\left(\frac{y}{\alpha}\right)^{\beta-1}\left[1+\left(\frac{y}{\alpha}\right)^{\beta}\right]^{-1} .
\end{gathered}
$$

A distribuição LL é caracterizada por modelar funções de taxa de falha decrescente e unimodal; isto é, quanto $\beta>1$ a função de taxa de falha tem forma unimodal, quando $\beta<1$ é decrescente e quando $\beta=1$ é decrescente iniciando $1 / \alpha$.

As propriedades básicas da distribuição log-logística são apresentadas por exemplo, por Kleiber e Kotz (2003), Lawless (2003) e Ashkar e Mahdi (2006). Os momentos são 
facilmente calculados (Tadikamalla e Johnson, 1982).

O r-ésimo momento para o tempo de falha é dado por

$$
E\left(Y^{r}\right)=\alpha^{r} \frac{r \pi / \beta}{\operatorname{sen}(\pi / \beta)}, \quad r<\beta .
$$

Assim, a esperança e a variância da distribuição LL são dadas pelas seguintes expressões

$$
E(Y)=\alpha \frac{\pi / \beta}{\operatorname{sen}(\pi / \beta)}, \quad \beta>1,
$$

$\mathrm{e}$

$$
\operatorname{Var}(Y)=\alpha^{2} \frac{2 \pi / \beta}{\operatorname{sen}(2 \pi / \beta)}-\left[\alpha \frac{\pi / \beta}{\operatorname{sen}(\pi / \beta)}\right]^{2}, \quad \beta>2,
$$

respectivamente. A função quantílica é dada por

$$
G^{-1}(u)=\alpha\left(\frac{u}{1-u}\right)^{1 / \beta}, \quad 0<u<1,
$$

em que $G^{-1}(u)$ é a inversa da fda da distribuição log-logística.

A Figura 2, mostra os gráficos da fdp, da função de sobrevivência e da função de taxa de falha, fixando $\alpha=25$ e para alguns valores dos parâmetros $\beta$. Note que a função de taxa de falha tem forma unimodal e decrescente.

A distribuição LL possui a mesma relação que a distribuição log-normal tem com a distribuição normal (Ahmad et al.(1988)), ou seja se $Y$ segue uma distribuição log-logística, então $T=\log (Y)$ segue uma distribuição logística, esta parte é abordada na Seção 4.1 deste trabalho.

Nos últimos anos, diversos trabalhos foram publicados utilizando a distribuição log-logística ou a distribuição logística, o que demonstra a importância dessas distribuições em diferentes áreas do conhecimento. A seguir é apresentado alguns desses trabalhos.

Ojo e Olapade (2003) definiram uma generalização da distribuição log-logística (beta log-logística), em que determinaram seus momentos e estabeleceram algumas relações entre a log-logística generalizada e outras distribuições.

Nesse mesmo ano, Leroy et al.(2003) investigaram o tempo de aparecimento de dentes permanentes de 4468 crianças em Franders (Bélgica), por meio da análise de sobrevivência, considerando a distribuição log-logística para o cálculo da mediana, média e intervalo de confiança. Indicando o aparecimento de dentes permanentes significativamente mais cedo em meninas do que em meninos.

No entanto, Ashkar e Mahdi (2006) investigaram o método dos momentos generalizados para estimação dos parâmetros do modelo log-logístico com dois parâmetros, ainda apresentaram uma aplicação na área de hidrologia, em estudos envolvendo baixo fluxo fluvial. 
(a)

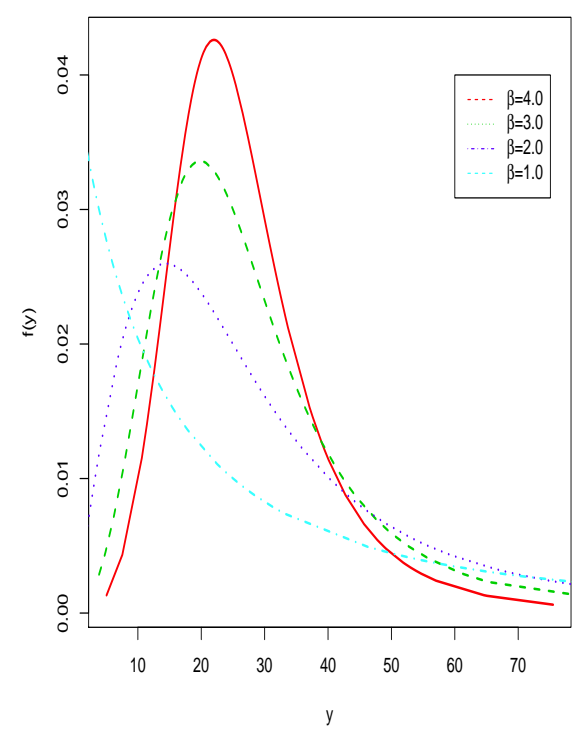

(b)

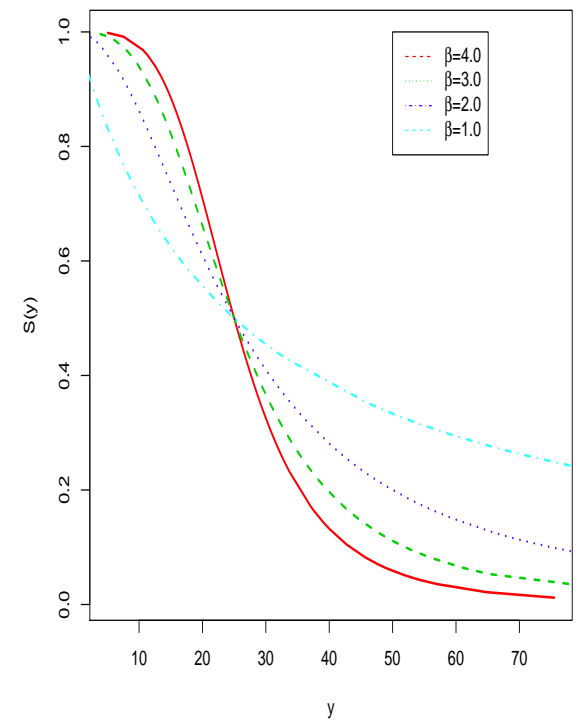

(c)

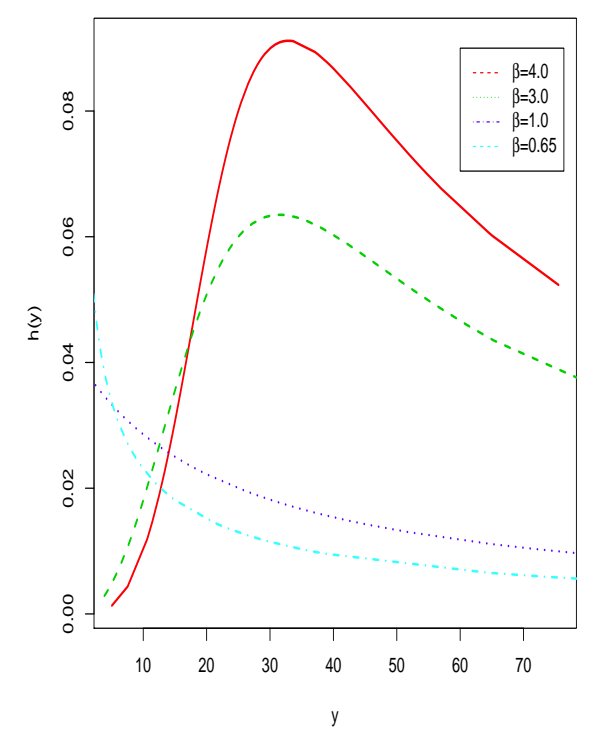

Figura 2 - Gráficos da função densidade (a), de sobrevivência (b) e de taxa de falha (c) da distribuição loglogística, para alguns valores do parâmetro $\beta$ fixando $\alpha=25$

Recentemente, Kirshenbaum et al. (2009) estudaram a recaída de dependentes químicos após um período inicial de abstinência. O objetivo principal do estudo foi verificar se a recaída de dependentes químicos exibe um padrão típico que pode ser caracterizado por uma função quantitativa comum. Os autores realizaram análises (utilizando as técnicas de análise de sobrevivência) em 20 estudos sobre indivíduos que permaneceram abstinentes depois de 
um período de abstinência inicial, onde observou-se que a distribuição log-logística foi a mais adequada para modelar os dados com maior precisão.

De Santana (2010), propôs uma nova distribuição utilizando a classe de distribuições proposta por Cordeiro e Castro (2011), para construir a nova distribuição de probabilidade denominada distribuição Kumaraswamy log-logística, a qual considera dois novos parâmetros, obtendo ganho nas formas da função de taxa de falha, que além de modelar dados em que a função de taxa de falha tem forma decrescente e unimodal, modela também forma crescente e forma de U.

Alternativamente, vários trabalhos introduziram as novas distribuições que são mais flexíveis em modelar funções de taxa de falha monótonas ou unimodales, mas eles não são utilizados para modelar taxas de falha em forma de $U$ ou de banheira; distribuições novas que foram construídas em termos de uma mistura de distribuições, assim tem-se, Proschan (1963) demonstrou que a função de taxa de falha decrescente é uma propriedade inerente à mistura de distribuições com funções de taxa de falha constante. Por outro lado, Adamidis e Loukas (1998) introduziram a distribuição Exponencial geométrica para modelar dados de tempo de vida com função taxa de falha decrescente.

Kus (2007) estudou uma nova distribuição com função taxa de falha decrescente, distribuição obtida pela mistura das distribuições exponencial e Poisson truncada. Carrasco et al. (2008a) propuseram a Weibull modificada generalizada com aplicações na análise de sobrevivência e Silva et al. (2010) estudaram em detalhe a distribuição beta Weibull modificada, a qual admite funções de taxa de falha crescente, decrescente, unimodal e forma de U. Seguindo a mesma idéia da distribuição EG, Silva et al. (2010) definiram a distribuição exponencial geométrica generalizada e demonstraram que a função taxa de falha pode ser crescente, decrescente ou unimodal. Uma extensão da distribuição EG é a distribuição Weibull geométrica, proposta por Barreto-Souza et al. (2011) o qual modela uma função taxa de falha monótona ou unimodal.

Considerando a idéia de Adamidis e Loukas (1998), Rodrigues (2011) propôs a distribuição Weibull binomial negativa (WBN) que é bastante flexível em análise de dados positivos e foram estudadas algumas de suas propriedades matemáticas. Esta é uma importante alternativa para os modelos Weibull e Weibull geométrica, que são sub-modelos da WBN. 


\subsection{Distribuição log-logística exponenciada}

A distribuição log-logística exponenciada foi proposta por Rosaiah et al. (2006). Tal distribuição é obtida da distribuição log-logística sugerida por Tadikamalla e Johnson (1982). A sua aplicabilidade foi também pelos primeiros autores, e vem sendo usada em estudos de planos de amostragem de aceitação baseados em testes de vida.

Seja $G(y)$ a função de distribuição acumulada da distribuição LL definida na equação (2), a função de distribuição acumulada da distribuição log-logística exponenciada (LLE) pode ser definida elevando $G(y)$ a potência $a$, isto é

$$
F(y)=G(y)^{a}=\left[1+\left(\frac{y}{\alpha}\right)^{-\beta}\right]^{-a}, \quad y>0 .
$$

Consequêntemente a função densidade de probabilidade da LLE com três parâmetros, $\alpha>0$, $\beta>0$ e $a>0$ é dada por

$$
f(y)=\frac{a \beta}{\alpha}\left(\frac{y}{\alpha}\right)^{\beta-1}\left[1+\left(\frac{y}{\alpha}\right)^{-\beta}\right]^{-(a-1)}\left[1+\left(\frac{y}{\alpha}\right)^{\beta}\right]^{-2}, y>0,
$$

em que, $\alpha$ é o parâmetro de escala, $\beta$ é o parâmetro de forma e $a$ é o segundo parâmetro de forma.

A Figura 3 mostra as formas gráficas da fdp da distribuição LLE, para diferentes valores dos parâmetros $\alpha, \beta$ e $a$ no qual pode-se observar a flexibilidade da fdp, devido a esses parâmetros. Assim, observa-se na Figura 3a o gráfico da fdp, considerando $\beta=3,5$ e variando os parâmetros $\alpha$ e $a$. Na figura 3b, mostra a fdp, fixando o parâmetro $\alpha=2,5$ e variando os parâmetros $\beta$ e $a$. Da mesma forma, na Figura 3c observa-se a fdp variando a e levando em consideração os parâmetros $\alpha=0,8$ e $\beta=3,5$.

Pode-se provar facilmente que a expressão (4) é uma fdp considerando a substituição $k=\left[1+\left(\frac{y}{\alpha}\right)^{-\beta}\right]^{-a}$. Se $Y$ é uma variável aleatória positiva com distribuição LLE e fdp dada na expressão (4) então, denota-se $Y \sim \operatorname{LLE}(\alpha, \beta, a)$.

A função de sobrevivência é dada por

$$
S(y)=1-F(y)=1-\left[1+\left(\frac{y}{\alpha}\right)^{-\beta}\right]^{-a} .
$$

Consequêntemente, a função de taxa de falha é dada por

$$
h(y)=\frac{a \beta}{\alpha}\left(\frac{y}{\alpha}\right)^{\beta-1}\left[1+\left(\frac{y}{\alpha}\right)^{-\beta}\right]^{-(a-1)}\left[1+\left(\frac{y}{\alpha}\right)^{\beta}\right]^{-2}\left\{1-\left[1+\left(\frac{y}{\alpha}\right)^{-\beta}\right]^{-a}\right\}^{-1} .
$$

A Figura 4 mostra os gráficos da função de sobrevivência da distribuição LLE para diferentes valores dos parâmetros $\alpha, \beta$ e $a$. A Figura 4a mostra para diferentes valores de 
(a)

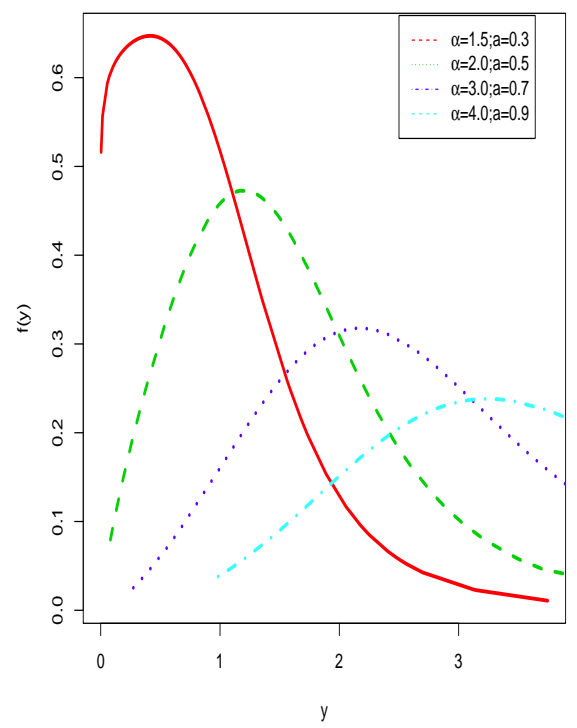

(b)

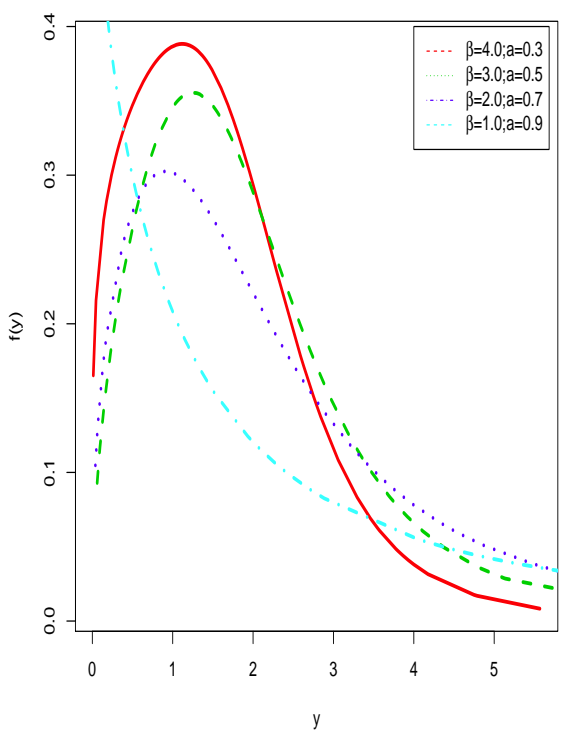

(c)

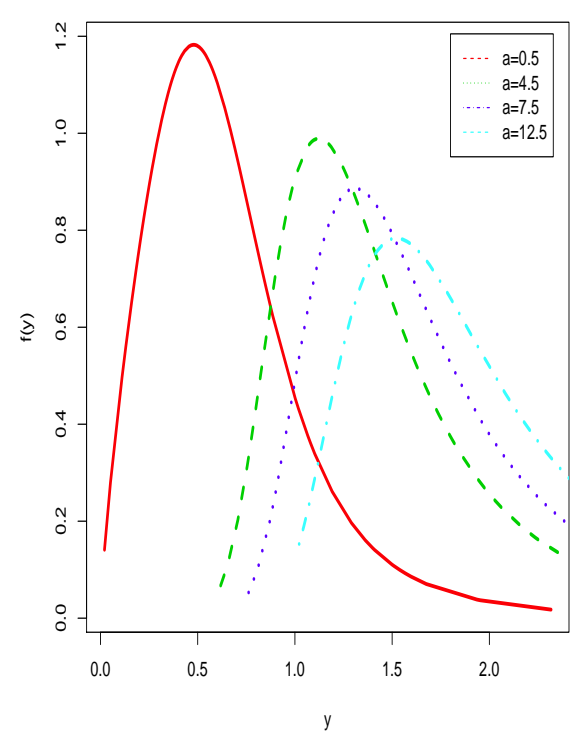

Figura 3 - Gráficos da fdp da distribuição LLE, para alguns valores dos parâmetros. (a) Para diferentes valores de $\alpha$ e $a$, fixando $\beta=3,5$. (b) Para diferentes valores de $\beta$ e $a$, fixando $\alpha=2,5$. (c) Para diferentes valores de $a$, fixando $\alpha=0,8$ e $\beta=3,5$

$\alpha$ e $a$, fixando $\beta=3,5$. Na Figura $4 \mathrm{~b}$ tem-se o gráfico fixando $\alpha=2,5$ para diferentes valores de $\beta$ e $a$, e na Figura 4c, pode-se observar o comportamento da função de sobrevivência para diferentes valores de $a$, considerando $\alpha=0,8$ e $\beta=3,5$.

Algumas formas da função de taxa de falha para alguns valores dos parâmetros 
(a)

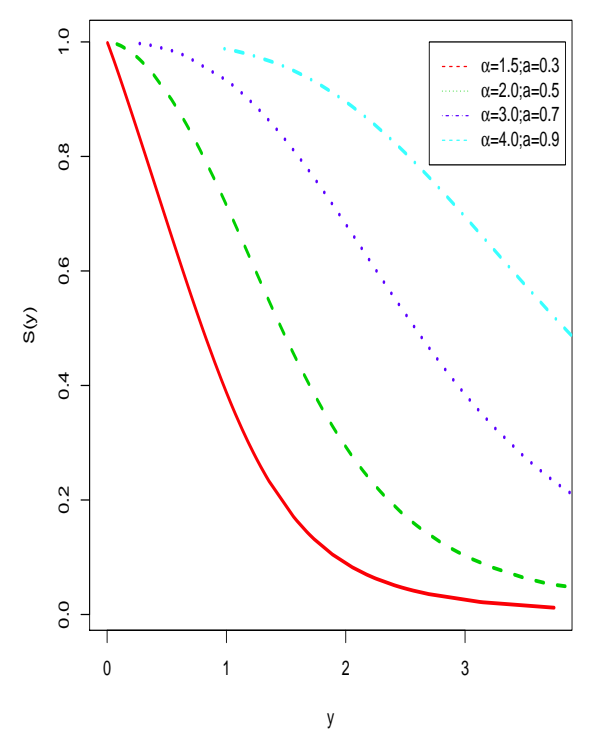

(b)

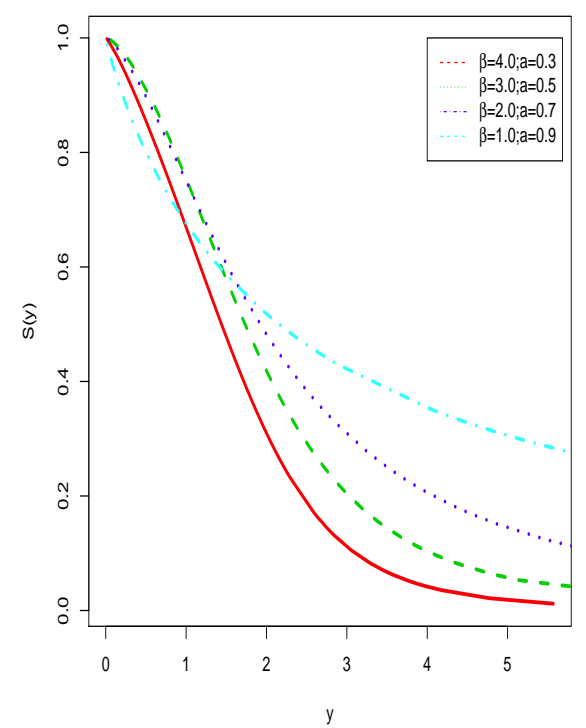

(c)

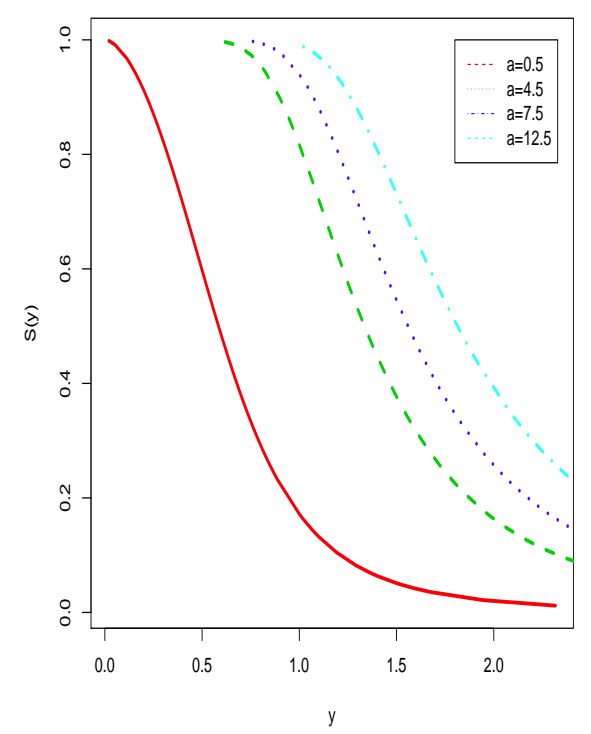

Figura 4 - Gráficos da função de sobrevivência da distribuição LLE, para alguns valores dos parâmetros. (a) Para diferentes valores de $\alpha$ e $a$, fixando $\beta=3,5$. (b) Para diferentes valores de $\beta$ e $a$, fixando $\alpha=2,5$. (c) Para diferentes valores de $a$, fixando $\alpha=0,8$ e $\beta=3,5$

$\alpha, \beta$ e $a$ da distribuição LLE, são dadas na Figura 5. Nas Figuras 5 a e 5b, apresenta-se a função de taxa de falha em forma unimodal quando $\beta>1$, além disso, na Figura 5b, mostra também forma decrescente quando $\beta<1$. Na Figura $5 \mathrm{c}$ a função decresce até certo ponto, e em seguida cresce até conseguir novamente a forma unimodal. 
A função quantílica é dada por

$$
G^{-1}(u)=\alpha\left(u^{-1 / a}-1\right)^{-1 / \beta}, \quad 0<u<1,
$$

em que $G^{-1}(u)$ é a inversa da fda da distribuição log-logística exponenciada.

(a)

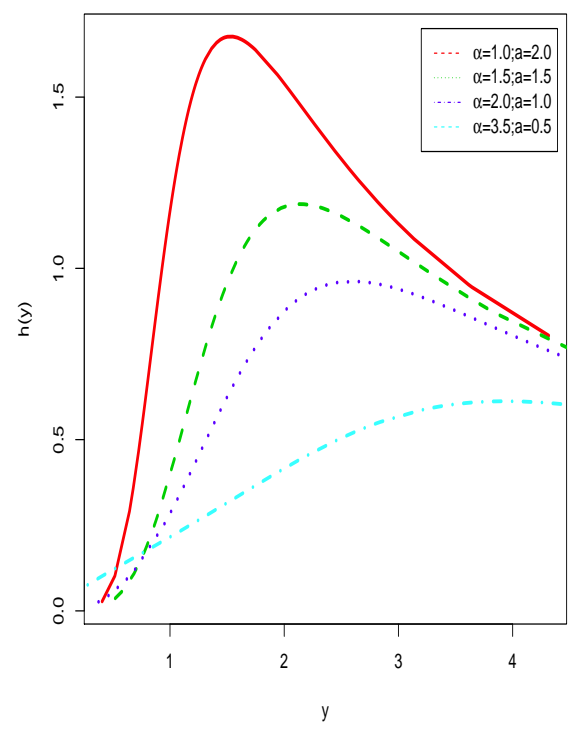

(b)

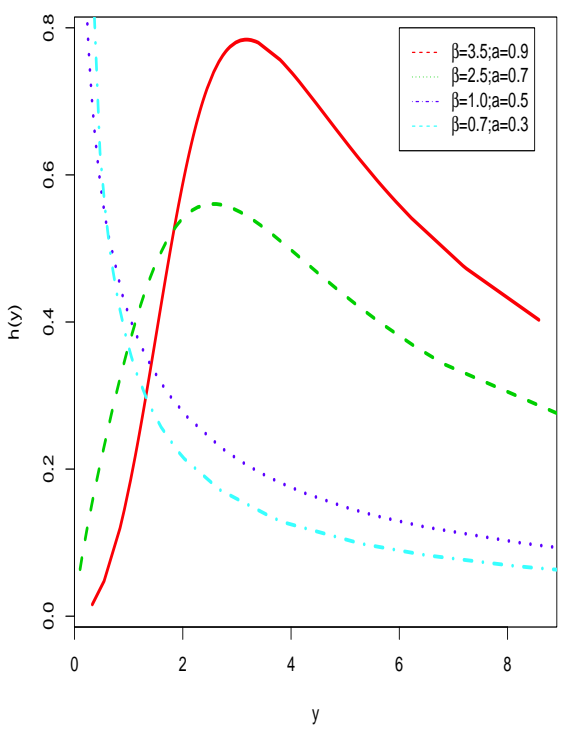

(c)

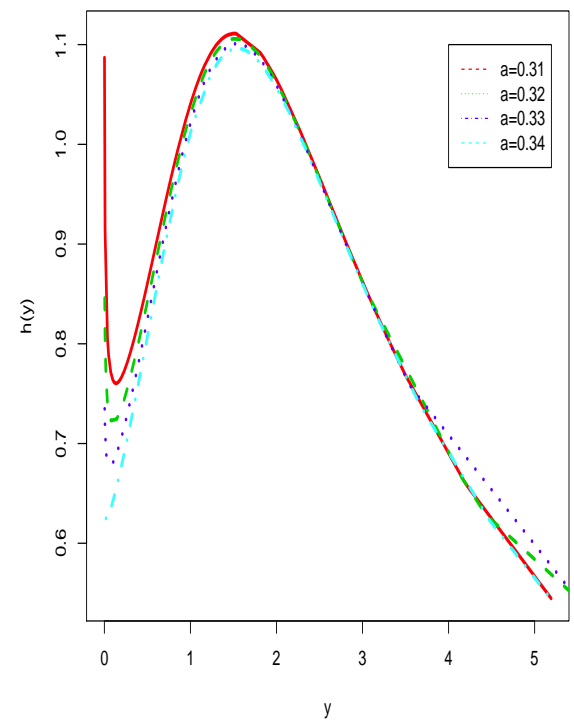

Figura 5 - Gráficos da função de taxa de falha da distribuição LLE, para alguns valores dos parâmetros. (a) Para diferentes valores de $\alpha$ e $a$, fixando $\beta=3,5$. (b) Para diferentes valores de $\beta$ e $a$, fixando $\alpha=2,5$. (c) Para diferentes valores de $a$, fixando $\alpha=1,7$ e $\beta=2,9$ 


\subsection{Distribuição log-logística exponenciada geométrica com dupla ativação}

Nesta seção, apresenta-se a definição e a construção da nova distribuição loglogística exponenciada geométrica baseada em uma dupla ativação.

\subsubsection{Distribuição log-logística exponenciada geométrica tipo I}

A nova distribuição que se propõe, será denominada distribuição log-logística exponenciada geométrica tipo I de 4 parâmetros, denotado por $\operatorname{LLEGI}(\alpha, \beta, a, p)$, como a mistura baseada na distribuição log-logística exponenciada e na distribuição geométrica. Assim, seja $Y_{1}, Y_{2}, \ldots, Y_{Z}$ uma amostra aleatória da distribuição log-logística exponenciada com fdp dada pela equação (4), $Z$ é outra variável aleatória discreta com distribuição de probabilidade dada por

$$
P(z ; p)=(1-p) p^{z-1}
$$

em que $Z \in \mathbb{N}$ e $p \in(0,1)$, assim $Z$ possui uma distribuição geométrica. Assumindo independência entre $Y_{1}, Y_{2}, \ldots, Y_{Z}$ e $Z$, define-se uma variável $X=\min \left(\left\{Y_{i}\right\}_{i=1}^{Z}\right)$, quer-se encontrar a função densidade de probabilidade marginal de $X$.

Utilizando a distribuição do mínimo para variáveis aleatórias contínuas independentes e identicamente distribuídas (iid), obtém-se a função de distribuição acumulada de $X$, o que pode ser derivado como a função de distribuição acumulada condicional de $X$ dado $Z$

$$
\begin{aligned}
F_{X \mid Z}(x \mid z) & =P(X \leq x \mid z) \\
& =P\left(\min \left(Y_{1}, Y_{2}, \ldots, Y_{z}\right) \leq x \mid z\right) \\
& =1-P\left(Y_{1}>x, Y_{2}>x, \ldots, Y_{z}>x\right) \\
& =1-\prod_{i=1}^{z} P\left(Y_{i}>x\right)
\end{aligned}
$$

por ser iid, a última expressão pode ser escrita como

$$
\begin{aligned}
F_{X \mid Z}(x \mid z) & =1-[P(Y>x)]^{z} \\
& =1-[1-P(Y \leq x)]^{z} \\
& =1-\left[1-F_{Y}(x)\right]^{z} .
\end{aligned}
$$

Diferenciando a expressão (6) em relação a $X$, obtém-se a função densidade de probabilidade de $X$ condicionada a $Z$

$$
f_{X \mid Z}(x \mid z)=z\left[1-F_{Y}(x)\right]^{z-1} f_{Y}(x) .
$$


Fazendo uso das expressões (7) e (5) e do fato que $X$ e $Z$ são independentes, obtém-se a função densidade de probabilidade conjunta de $(X, Z)$

$$
f(x, z)=f_{X \mid Z}(x) P(z ; p)=z f_{Y}(x)\left[1-F_{Y}(x)\right]^{z-1}(1-p) p^{z-1}
$$

Somando a expressão (8) para z variando de 1 até $\infty$, obtém-se a função densidade de probabilidade marginal de $X$

$$
\begin{aligned}
f(x) & =\sum_{z=1}^{\infty} f(x, z) \\
& =(1-p) f_{Y}(x) \sum_{z=1}^{\infty} z\left\{\left[1-F_{Y}(x)\right] p\right\}^{z-1} .
\end{aligned}
$$

Considerando a expressão $\sum_{z=1}^{\infty} z\left\{\left[1-F_{Y}(x)\right] p\right\}^{z-1}$ como a derivada de uma série geométrica, obtém-se finalmente a função densidade marginal de $X$

$$
f(x)=(1-p) f_{Y}(x)\left\{1-p\left[1-F_{Y}(x)\right]\right\}^{-2},
$$

a qual será denominado como a fdp para o mínimo. Integrando a equação (9), de $-\infty$ até $x$, obtém-se a função de distribuição acumulada de $X$

$$
F(x)=F_{Y}(x)\left\{1-p\left[1-F_{Y}(x)\right]\right\}^{-1},
$$

que será denominado como a fda para o mínimo. Finalmente, substituindo na equação (9), $F_{Y}(x)$ e $f_{Y}(x)$ pela fda e fdp da distribuição log-logística exponenciada respectivamente, obtémse, a distribuição log-logística exponenciada geométrica tipo I com quatro parâmetros, denotada por $\operatorname{LLEGI}(\alpha, \beta, a, p)$, em que a fdp e fda são definidas pelas equações (11) e (12) respectivamente,

$$
\begin{gathered}
f(x ; \alpha, \beta, a, p)=(1-p) \frac{a \beta}{\alpha}\left(\frac{x}{\alpha}\right)^{\beta-1}\left[1+\left(\frac{x}{\alpha}\right)^{-\beta}\right]^{-(a-1)}\left[1+\left(\frac{x}{\alpha}\right)^{\beta}\right]^{-2} \times \\
\\
\left\{1-p\left[1-\left[1+\left(\frac{x}{\alpha}\right)^{-\beta}\right]^{-a}\right]\right\}^{-2}, \quad x>0 . \\
F(x ; \alpha, \beta, a, p)=\left[1+\left(\frac{x}{\alpha}\right)^{-\beta}\right]^{-a}\left\{1-p\left[1-\left[1+\left(\frac{x}{\alpha}\right)^{-\beta}\right]^{-a}\right]\right\}^{-1}, \quad x>0 .
\end{gathered}
$$

Pode-se provar que a expressão (11) é uma função densidade considerando a substituição $k=\left\{1-p\left[1-\left[1+\left(\frac{x}{\alpha}\right)^{-\beta}\right]^{-a}\right]\right\}^{-1}$. 
Os gráficos da fdp da distribuição LLEGI são apresentados na Figura 6, para alguns valores fixados dos parâmetros $\alpha$ e $\beta$ e variando os valores dos parâmetros $a$ e $p$, onde observa-se uma flexibilidade da fdp, devido a esses parâmetros. Uma importante propriedade da distribuição LLEGI é o fato de possuir alguns sub modelos tais como

- Se $a=1$, obtêm-se a distribuição log-logística geométrica tipo I.

$$
f(x ; \alpha, \beta, a=1, p)=(1-p) \frac{\beta}{\alpha}\left(\frac{x}{\alpha}\right)^{\beta-1}\left[1+\left(\frac{x}{\alpha}\right)^{\beta}\right]^{-2}\left\{1-p\left[1-\left[1+\left(\frac{x}{\alpha}\right)^{-\beta}\right]^{-1}\right]\right\}^{-2} .
$$

- Se $p=0$, obtêm-se a distribuição log-logística exponenciada.

$$
f(x ; \alpha, \beta, a, p=0)=\frac{a \beta}{\alpha}\left(\frac{x}{\alpha}\right)^{\beta-1}\left[1+\left(\frac{x}{\alpha}\right)^{-\beta}\right]^{-(a-1)}\left[1+\left(\frac{x}{\alpha}\right)^{\beta}\right]^{-2} .
$$

- Se $a=1$ e $p=0$, obtêm-se a distribuição log-logística.

$$
f(x ; \alpha, \beta, a=1, p=0)=\frac{\beta}{\alpha}\left(\frac{x}{\alpha}\right)^{\beta-1}\left[1+\left(\frac{x}{\alpha}\right)^{\beta}\right]^{-2} .
$$

Se $X$ segue uma distribuição LLEGI correspondente à expressão (11), a função de sobrevivência é expressa por

$$
S(x)=1-\left[1+\left(\frac{x}{\alpha}\right)^{-\beta}\right]^{-a}\left\{1-p\left[1-\left[1+\left(\frac{x}{\alpha}\right)^{-\beta}\right]^{-a}\right]\right\}^{-1}, \quad x>0,
$$

e a função taxa de falha, é dada por

$$
\begin{aligned}
h(x)= & \frac{(1-p) a \beta}{\alpha}\left(\frac{x}{\alpha}\right)^{\beta-1}\left[1+\left(\frac{x}{\alpha}\right)^{-\beta}\right]^{-(a-1)}\left[1+\left(\frac{x}{\alpha}\right)^{\beta}\right]^{-2} \times \\
& \left\{1-p\left[1-\left[1+\left(\frac{x}{\alpha}\right)^{-\beta}\right]^{-a}\right]\right\}^{-2} \times \\
& \left\{1-\left[1+\left(\frac{x}{\alpha}\right)^{-\beta}\right]^{-a}\left\{1-p\left[1-\left[1+\left(\frac{x}{\alpha}\right)^{-\beta}\right]^{-a}\right]\right\}^{-1}\right\}^{-1} .
\end{aligned}
$$

O comportamento da função de taxa de falha da distribuição LLEGI é apresentado na Figura 7, para alguns valores fixados dos parâmetros $\alpha$ e $\beta$ e variando os valores dos parâmetros $a$ e $p$. Pode-se observar na Figura 7a, que a função de taxa de falha apresenta formas monótonas, a Figura 7b, mostra as formas unimodais e na Figura 7c mostra a forma bimodal.

Para simular dados de uma distribuição LLEGI, utilizou-se a inversa da fda correspondente à expressão (12)

$$
Q(u)=F^{-1}(u)=\alpha\left\{\left[\frac{1-u p}{u(1-p)}\right]^{\frac{1}{a}}-1\right\}^{-\frac{1}{\beta}},
$$

em que $u$ tem distribuição uniforme $(0,1)$. 
(a)

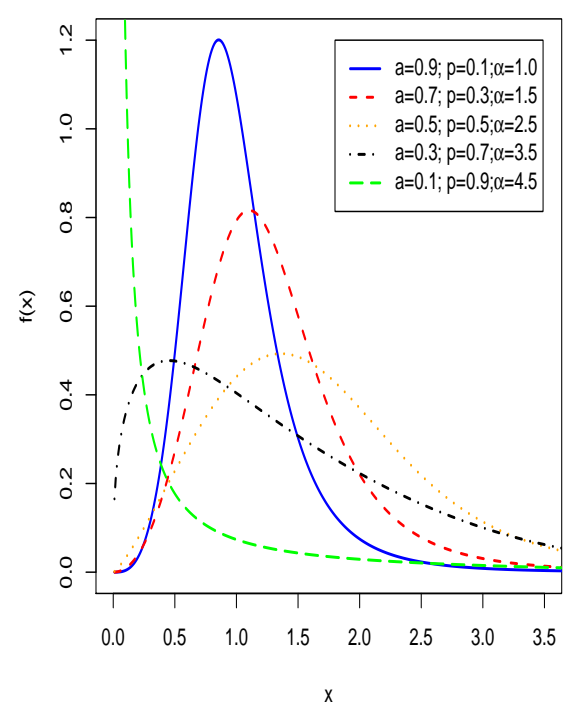

(b)

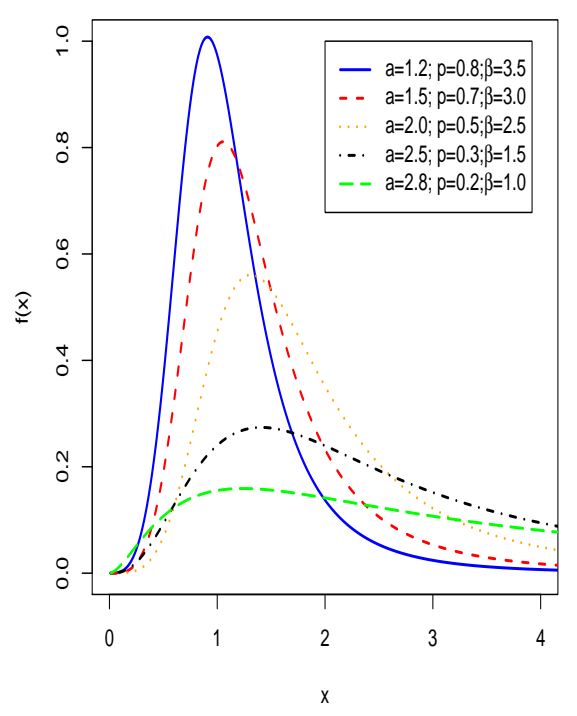

(c)

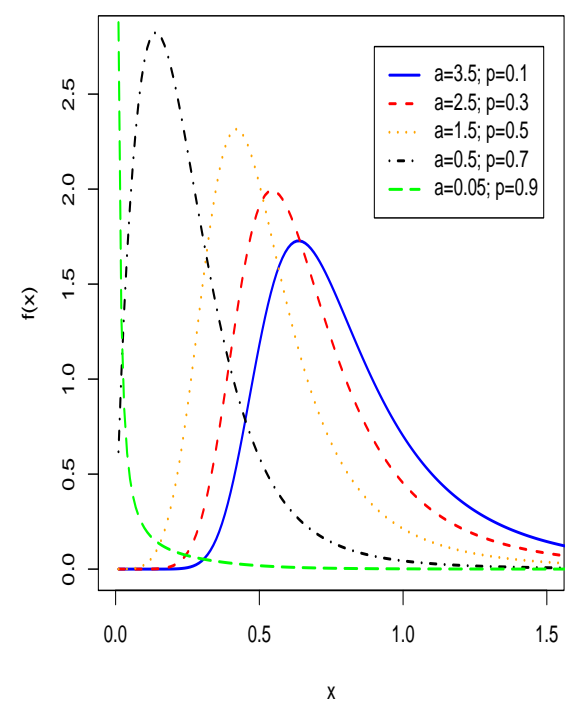

Figura 6 - Gráficos da fdp da distribuição LLEGI, para alguns valores dos parâmetros. (a) Para diferentes valores de $a, p$ e $\alpha$, fixando $\beta=4,5$. (b) Para diferentes valores de $a, p$ e $\beta$, fixando $\alpha=1,5$. (c) Para diferentes valores de $a$ e $p$, fixando $\alpha=0,5$ e $\beta=3,5$

\subsubsection{Distribuição log-logística exponenciada geométrica tipo II}

Considerando a mesma idéia da composição de uma distribuição log-logística exponenciada geométrica tipo I, em que foi considerado como valor mínimo uma variável geométrica, agora será feita a composição da distribuição que será denominada distribuição 
(a)

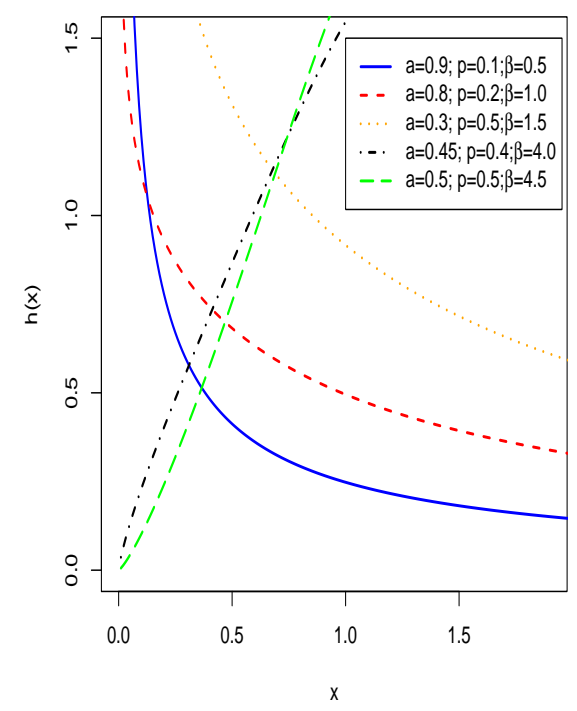

(b)

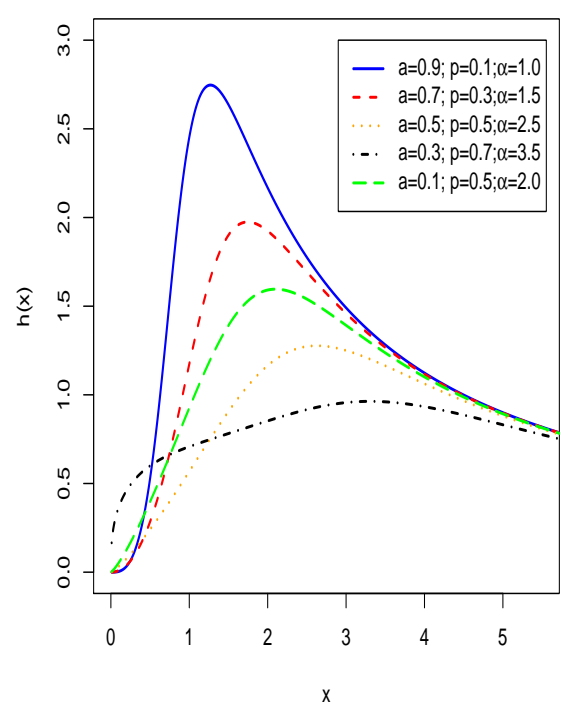

(c)

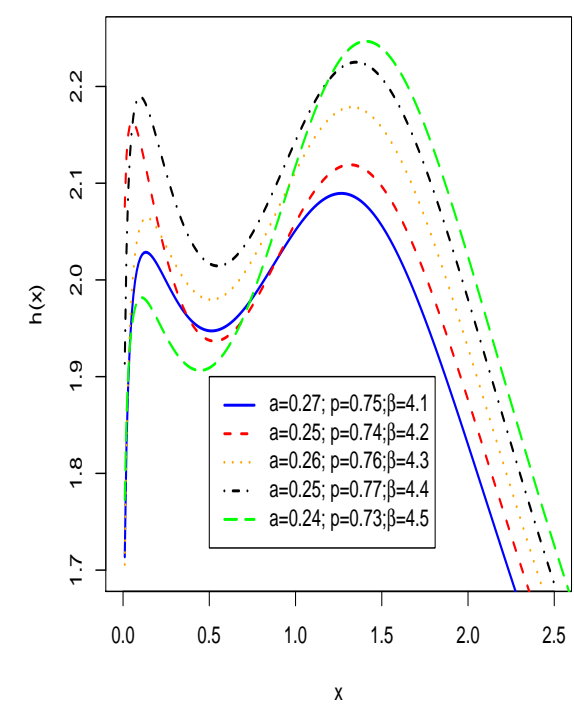

Figura 7 - Gráficos da função de taxa de falha da distribuição LLEGI, para alguns valores dos parâmetros. (a) Para diferentes valores de $a, p$ e $\beta$, fixando $\alpha=1,5$. (b) Para diferentes valores de $a, p$ e $\alpha$, fixando $\beta=4,5$. (c) Para diferentes valores de $a, p$ e $\beta$, fixando $\alpha=1,5$

log-logística exponenciada geométrica tipo II, denotado por LLEGII no qual será utilizado como valor máximo uma variável geométrica.

Seja $Y_{1}, Y_{2}, \ldots, Y_{Z}$ uma amostra aleatória da distribuição log-logística exponenciada com densidade dada pela equação (4) e $Z$ é outra variável aleatória com distribuição 
geométrica expressa pela equação (5). Assumindo independência entre $Y_{1}, Y_{2}, \ldots, Y_{Z}$ e $Z$, define-se uma variável $X=\max \left(\left\{Y_{i}\right\}_{i=1}^{Z}\right)$, quer-se encontrar a função densidade de probabilidade marginal de $X$.

Utilizando a distribuição do máximo para variáveis aleatórias contínuas independentes e identicamente distribuidas (iid), obtém-se a função de distribuição acumulada de $X$, o que pode ser derivado como a função de distribuição acumulada condicional de $X$ dado $Z$

$$
\begin{aligned}
F_{X \mid Z}(x \mid z) & =P(X \leq x \mid z) \\
& =P\left(\max \left(Y_{1}, Y_{2}, \ldots, Y_{z}\right) \leq x \mid z\right) \\
& =P\left(Y_{1} \leq x, Y_{2} \leq x, \ldots, Y_{z} \leq x\right) \\
& =\prod_{i=1}^{z} P\left(Y_{i} \leq x\right),
\end{aligned}
$$

por ser iid, a última expressão pode ser escrita como

$$
\begin{aligned}
F_{X \mid Z}(x \mid z) & =[P(Y \leq x)]^{z} \\
& =\left[F_{Y}(x)\right]^{z} .
\end{aligned}
$$

Diferenciando a expressão (13) em relação a $X$, obtém-se a função densidade de probabilidade de $X$ condicionada a $Z$

$$
f_{X \mid Z}(x \mid z)=z\left[F_{Y}(x)\right]^{z-1} f_{Y}(x) .
$$

Fazendo uso das expressões (14) e (5), e do fato que $X$ e $Z$ são independentes, obtém-se a função densidade de probabilidade conjunta de $(X, Z)$

$$
f(x, z)=f_{X \mid Z}(x) P(z ; p)=z f_{Y}(x)\left[F_{Y}(x)\right]^{z-1}(1-p) p^{z-1} .
$$

Somando a expressão (15) para $z$ variando de 1 até $\infty$, obtém-se a função densidade de probabilidade marginal de $X$

$$
\begin{aligned}
f(x) & =\sum_{z=1}^{\infty} f(x, z) \\
& =(1-p) f_{Y}(x) \sum_{z=1}^{\infty} z\left\{\left[F_{Y}(x)\right] p\right\}^{z-1} .
\end{aligned}
$$

Considerando a expressão $\sum_{z=1}^{\infty} z\left\{\left[F_{Y}(x)\right] p\right\}^{z-1}$ como a derivada de uma série geométrica, obtém-se finalmente a função densidade marginal de $X$

$$
f(x)=(1-p) f_{Y}(x)\left\{1-p\left[F_{Y}(x)\right]\right\}^{-2},
$$


a qual será denominada como a fdp para o máximo.

Integrando a equação (16), de $-\infty$ até $x$, obtém-se a função de distribuição acumulada de $X$

$$
F(x)=(1-p) F_{Y}(x)\left\{1-p\left[F_{Y}(x)\right]\right\}^{-1},
$$

que será denominado como a fda para o máximo. Finalmente, substituindo na equação (16), $F_{Y}(x)$ e $f_{Y}(x)$ pela fda e fdp da distribuição log-logística exponenciada, respectivamente obtém-se, a distribuição log-logística exponenciada geométrica tipo II com quatro parâmetros, denotada por $\operatorname{LLEGII}(\alpha, \beta, a, p)$ cujas fdp e fda são definidas pelas equações (18) e (19) respectivamente,

$$
\begin{aligned}
& f(x ; \alpha, \beta, a, p)=(1-p) \frac{a \beta}{\alpha}\left(\frac{x}{\alpha}\right)^{\beta-1}\left[1+\left(\frac{x}{\alpha}\right)^{-\beta}\right]^{-(a-1)}\left[1+\left(\frac{x}{\alpha}\right)^{\beta}\right]^{-2} \times \\
&\left\{1-p\left[1+\left(\frac{x}{\alpha}\right)^{-\beta}\right]^{-a}, \quad x>0 .\right. \\
& F(x ; \alpha, \beta, a, p)=(1-p)\left[1+\left(\frac{x}{\alpha}\right)^{-\beta}\right]^{-a}\left\{1-p\left[1+\left(\frac{x}{\alpha}\right)^{-\beta}\right]^{-a}, \quad x>0 .\right.
\end{aligned}
$$

Pode-se provar que a expressão (18) é uma função densidade considerando a substituição $k=\left\{1-p\left[1+\left(\frac{x}{\alpha}\right)^{-\beta}\right]^{-a}\right\}^{-1}$.

Observa-se que alguns sub-modelos da distribuição LLEGII são apresentados na Figura 8, para alguns valores fixados dos parâmetros $\alpha$ e $\beta$ e variando os valores dos parâmetros $a$ e $p$, em que observa-se uma flexibilidade da fdp, devido a esses parâmetros.

Uma importante propriedade da distribuição LLEGII é o fato de possuir alguns sub-modelos particulares tais como

- Se $a=1$, obtêm-se a distribuição log-logística geométrica tipo II.

$$
f(x ; \alpha, \beta, a=1, p)=(1-p) \frac{\beta}{\alpha}\left(\frac{x}{\alpha}\right)^{\beta-1}\left[1+\left(\frac{x}{\alpha}\right)^{\beta}\right]^{-2}\left\{1-p\left[1+\left(\frac{x}{\alpha}\right)^{-\beta}\right]^{-1}\right\}^{-2} .
$$

- Se $p=0$, obtêm-se a distribuição log-logística exponenciada.

$$
f(x ; \alpha, \beta, a, p=0)=\frac{a \beta}{\alpha}\left(\frac{x}{\alpha}\right)^{\beta-1}\left[1+\left(\frac{x}{\alpha}\right)^{-\beta}\right]^{-(a-1)}\left[1+\left(\frac{x}{\alpha}\right)^{\beta}\right]^{-2} .
$$

- Se $a=1$ e $p=0$, obtêm-se a distribuição log-logística.

$$
f(x ; \alpha, \beta, a=1, p=0)=\frac{\beta}{\alpha}\left(\frac{x}{\alpha}\right)^{\beta-1}\left[1+\left(\frac{x}{\alpha}\right)^{\beta}\right]^{-2} .
$$


Se $X$ segue uma distribuição LLEGII correspondente à expressão (18), a função de sobrevivência é expressa por

$$
S(x)=1-(1-p)\left[1+\left(\frac{x}{\alpha}\right)^{-\beta}\right]^{-a}\left\{1-p\left[1+\left(\frac{x}{\alpha}\right)^{-\beta}\right]^{-a}\right\}^{-1}, \quad x>0
$$

e a função taxa de falha, é dada por

$$
h(x)=\frac{(1-p) \frac{a \beta}{\alpha}\left(\frac{x}{\alpha}\right)^{\beta-1}\left[1+\left(\frac{x}{\alpha}\right)^{-\beta}\right]^{-(a-1)}\left[1+\left(\frac{x}{\alpha}\right)^{\beta}\right]^{-2}\left\{1-p\left[1+\left(\frac{x}{\alpha}\right)^{-\beta}\right]^{-a}\right\}^{-2}}{1-(1-p)\left[1+\left(\frac{x}{\alpha}\right)^{-\beta}\right]^{-a}\left\{1-p\left[1+\left(\frac{x}{\alpha}\right)^{-\beta}\right]^{-a}\right\}^{-1}} .
$$

A Figura 9 mostra o comportamento da função taxa de falha da distribuição LLEGII fixando os parâmetros $\alpha$ e $\beta$, para diferentes valores de $a$ e $p$. Observa-se por meio dos gráficos que a função taxa de falha apresenta forma unimodal (9.a), forma de banheira (9.b) e forma bimodal (9.c)

Para simular dados de uma distribuição LLEGII utilizou-se a inversa da fda correspondente à expressão (19)

$$
Q(u)=F^{-1}(u)=\alpha\left\{\left[\frac{(1-p)+u p}{u}\right]^{\frac{1}{a}}-1\right\}^{-\frac{1}{\beta}}
$$

em que $u$ tem distribuição uniforme $(0,1)$.

\subsection{Propriedades da log-logística exponenciada geométrica}

Uma prática habitual em uma análise estatística, é descrever uma distribuição por meio de algumas características importantes da mesma, que podem ser estudadas por meio dos momentos (por exemplo, tendência, dispersão, assimetria e curtose). Nas próximas seções apresentam-se algumas propriedades da distribuição LLEG. Na Seção 3.4.1, será apresentada para a fdp em expansão de série de potências para as distribuições LLEGI e LLGEII. Os momentos de probabilidade ponderada e a função geradora de momentos serão apresentadas nas Seções 3.4.2 e 3.4.3, respectivamente.

\subsubsection{Expansão da função densidade da distribuição log-logística exponenciada geométrica}

\section{- Distribuição LLEGI}

Pode-se escrever a fdp da distribuição da LLEGI dada pela expressão (11), como uma combinação de distribuições log-logísticas exponenciadas. Partindo da expressão (11), o 
(a)

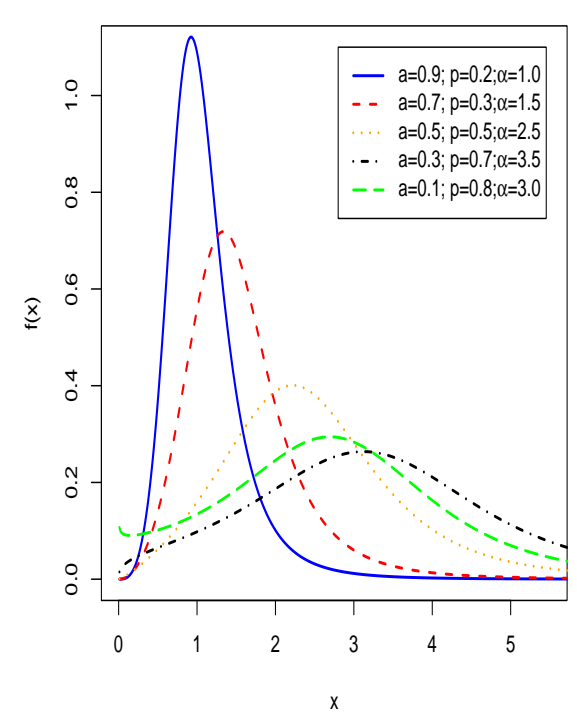

(b)

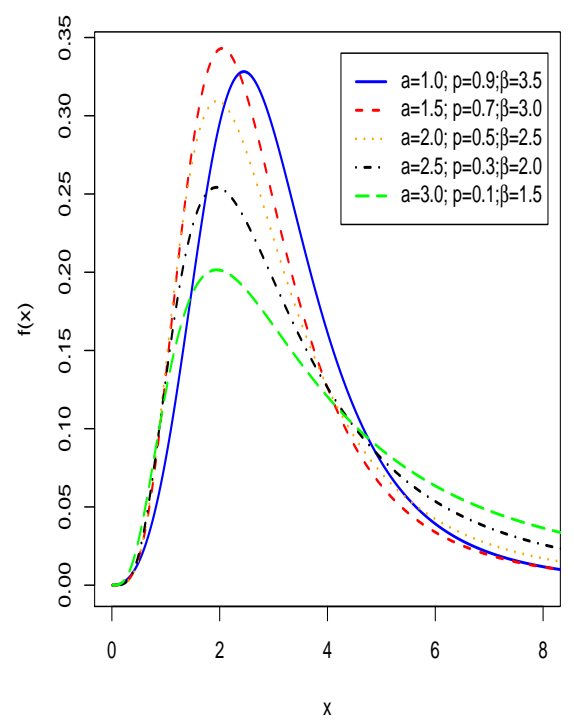

(c)

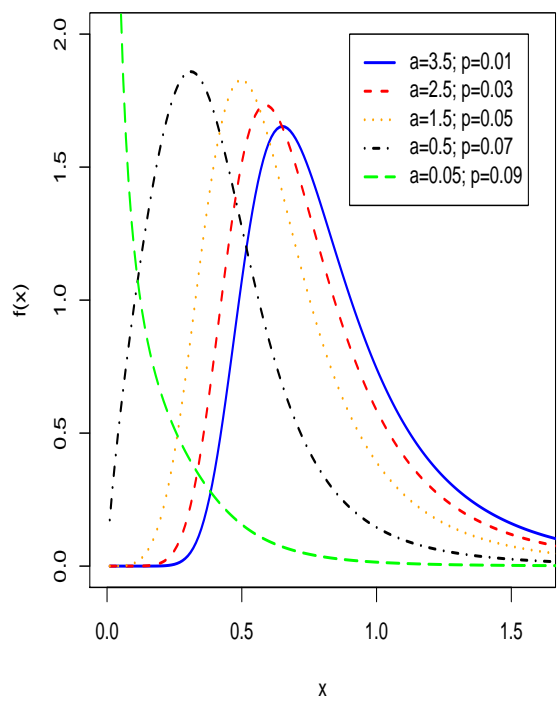

Figura 8 - Gráficos da fdp da distribuição LLEGII, para alguns valores dos parâmetros. (a) Para diferentes valores de $a, p$ e $\alpha$, fixando $\beta=4,5$. (b) Para diferentes valores de $a, p$ e $\beta$, fixando $\alpha=1,5$. (c) Para diferentes valores de $a$ e $p$, fixando $\alpha=0,5$ e $\beta=3,5$

termo que está elevado à potência -2 , pode ser escrito como sendo $z=1-F_{\alpha, \beta, a}(x)$, em que $F_{\alpha, \beta, a}(x)$ é a fda da LLE, dada pela equação (3) em termos da variável $X$, assim

$$
F_{\alpha, \beta, a}(x)=\left[1+\left(\frac{x}{\alpha}\right)^{-\beta}\right]^{-a}
$$


(a)

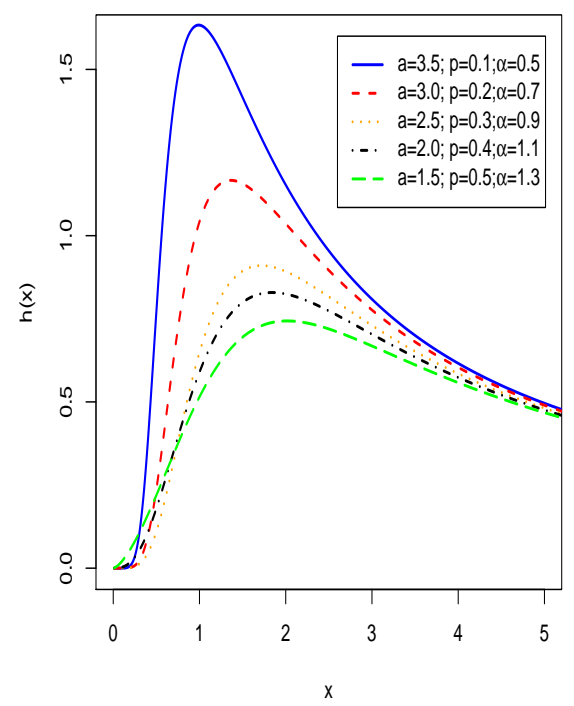

(b)

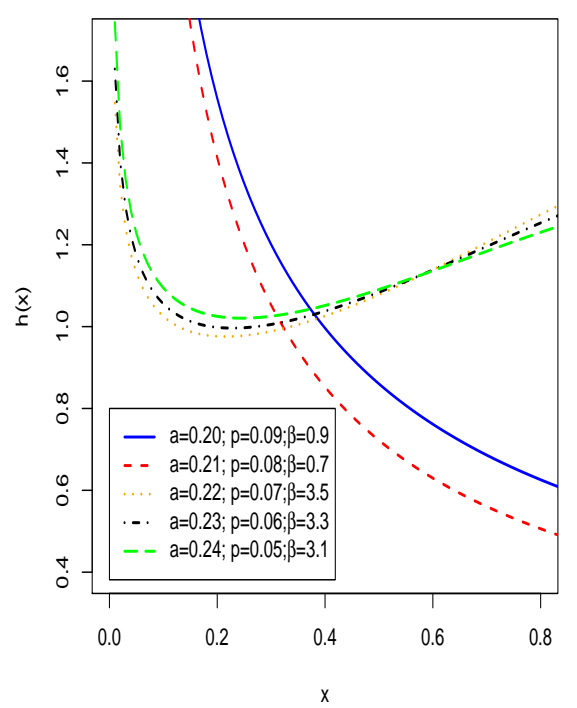

(c)

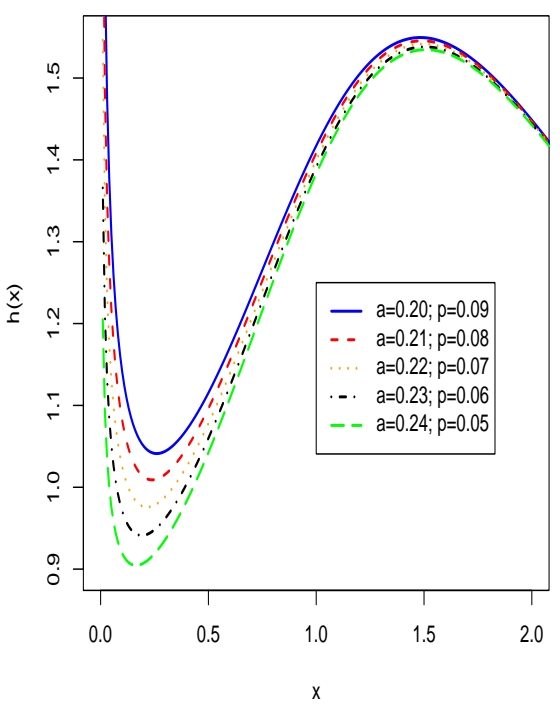

Figura 9 - Gráficos da taxa de falha da distribuição LLEGII, para alguns valores dos parâmetros. (a) Para diferentes valores de $a, p$ e $\alpha$, fixando $\beta=2,5$. (b) Para diferentes valores de $a, p$ e $\beta$, fixando $\alpha=1,5$. (c) Para diferentes valores de $a$ e $p$, fixando $\alpha=1,5$ e $\beta=3,5$

Considerando a seguinte expressão $\left(\frac{1-z}{1-p z}\right)^{2}$, e levando em conta que $0<p z<1$, então expandindo $(1-p z)^{-2}$ em série de potências e usando a forma

$$
(1-z)^{-k}=\sum_{j=0}^{\infty} \frac{\Gamma(k+j)}{\Gamma(k) j !} z^{j}
$$


obtém-se

$$
(1-p z)^{-2}=\sum_{j=0}^{\infty} \frac{\Gamma(j+2)}{\Gamma(2) j !}(p z)^{j} .
$$

Substituindo $z=1-F_{\alpha, \beta, a}(x)$ em (21), obtém-se

$$
(1-p z)^{-2}=\sum_{j=0}^{\infty}(j+1) p^{j}\left[1-F_{\alpha, \beta, a}(x)\right]^{j} .
$$

Por outro lado, substituindo $F_{\alpha, \beta, a}(x)$ em (22)

$$
(1-p z)^{-2}=\sum_{j=0}^{\infty}(j+1) p^{j}\left\{1-\left[1+\left(\frac{x}{\alpha}\right)^{-\beta}\right]^{-a}\right\}^{j},
$$

utilizando a (23) na expressão de (11) da densidade LLEGI, obtém-se

$$
\begin{aligned}
f_{\alpha, \beta, a, p}(x)= & (1-p) \frac{a \beta}{\alpha}\left(\frac{x}{\alpha}\right)^{\beta-1}\left[1+\left(\frac{x}{\alpha}\right)^{-\beta}\right]^{-(a-1)}\left[1+\left(\frac{x}{\alpha}\right)^{\beta}\right]^{-2} \times \\
& \left.\left.\left\{1-p\left[1-\left[1+\left(\frac{x}{\alpha}\right)^{-\beta}\right]^{-a}\right]\right\}^{-2} \times\right]^{-\beta}\right]^{-(a-1)}\left[1+\left(\frac{x}{\alpha}\right)^{\beta}\right]^{-2} \times \\
= & (1-p) \frac{a \beta}{\alpha}\left(\frac{x}{\alpha}\right)^{\beta-1}\left[1+\left(\frac{x}{\alpha}\right)^{-2}\right\}^{j}\left[1-\left[1+\left(\frac{x}{\alpha}\right)^{-\beta}\right]^{-a}\right\}^{\infty} \sum_{j=0}^{\infty}(j+1) p^{j}\left\{1+\left(\frac{x}{\alpha}\right)^{\beta} \times\right. \\
= & \frac{a \beta}{\alpha}\left(\frac{x}{\alpha}\right)^{\beta-1}\left[1+\left(\frac{x}{\alpha}\right)^{-\beta}\right]^{-(a-1)}\left[1-\left[1+\left(\frac{x}{\alpha}\right)^{-\beta}\right\}^{-a} .\right. \\
& \sum_{j=0}^{\infty}(j+1)(1-p) p^{j}\{1-[1
\end{aligned}
$$

Levando em consideração a fdp da distribuição LLE dada por

$$
f_{\alpha, \beta, a}(x)=\frac{a \beta}{\alpha}\left(\frac{x}{\alpha}\right)^{\beta-1}\left[1+\left(\frac{x}{\alpha}\right)^{-\beta}\right]^{-(a-1)}\left[1+\left(\frac{x}{\alpha}\right)^{\beta}\right]^{-2},
$$

obtém-se a expansão para a densidade da LLEGI, sendo uma combinação linear das distribuições log-logísticas exponenciadas. A expansão da fdp da LLEGI é dada por

$$
f_{\alpha, \beta, a, p}(x)=f_{\alpha, \beta, a}(x) \sum_{j=0}^{\infty} w_{j}\left[1-F_{\alpha, \beta, a}(x)\right]^{j},
$$

a expressão $\left[1-F_{\alpha, \beta, a}(x)\right]^{j}$ pode estar representada em termos dos coeficientes binomiais, como $j$ é um inteiro, então pode-se escrever como

$$
\left[1-F_{\alpha, \beta, a}(x)\right]^{j}=\sum_{i=0}^{j}(-1)^{i}\left(\begin{array}{l}
j \\
i
\end{array}\right) F_{\alpha, \beta, a}(x)^{i}
$$


então a fdp da expressão (25) é expressa por

$$
f_{\alpha, \beta, a, p}(x)=(1-p) f_{\alpha, \beta, a}(x) \sum_{j=0}^{\infty} \sum_{i=0}^{j} w_{j}(-1)^{i}\left(\begin{array}{l}
j \\
i
\end{array}\right) F_{\alpha, \beta, a}(x)^{i} .
$$

Considerando $w_{j}=(j+1) p^{j}$ da expressão (26) como os pesos ponderados que multiplicam a família das distribuições log-logísticas exponenciadas.

\section{- Distribuição LLEGII}

Seguindo a mesma idéia da construção da expansão da função da distribuição LLEGI, pode-se escrever a fdp da distribuição LLEGII como uma combinação de distribuições LLE. Neste caso, partindo da expressão (18), o termo que está elevado à potência -2, pode ser escrito como sendo $z=F_{\alpha, \beta, a}(x)$, em que $F_{\alpha, \beta, a}(x)$ é a fda da LLE, dada pela

equação (3) em termos da variável $X$, considerando a seguinte expressão $\left(\frac{1-z}{1-p z}\right)^{2}$, e levando em conta que $0<p z<1$, então expandindo $(1-p z)^{-2}$ em série de potências da forma (21), obtém-se a equação

$$
(1-p z)^{-2}=\sum_{j=0}^{\infty} \frac{\Gamma(j+2)}{\Gamma(2) j !}(p z)^{j},
$$

substituindo $z=F_{\alpha, \beta, a}(x)$ em (27), obtém-se

$$
(1-p z)^{-2}=\sum_{j=0}^{\infty}(j+1) p^{j}\left[F_{\alpha, \beta, a}(x)\right]^{j} .
$$

Por outro lado, substituindo $F_{\alpha, \beta, a}(x)$ em (28), tem-se

$$
(1-p z)^{-2}=\sum_{j=0}^{\infty}(j+1) p^{j}\left[1+\left(\frac{x}{\alpha}\right)^{-\beta}\right]^{-a j},
$$

utilizando a (29) na expressão de (18) da densidade LLEGII, obtém-se

$$
\begin{aligned}
& f_{\alpha, \beta, a, p}(x)=(1-p) \frac{a \beta}{\alpha}\left(\frac{x}{\alpha}\right)^{\beta-1}\left[1+\left(\frac{x}{\alpha}\right)^{-\beta}\right]^{-(a-1)}\left[1+\left(\frac{x}{\alpha}\right)^{\beta}\right]^{-2} \times \\
& \left\{1-p\left[1+\left(\frac{x}{\alpha}\right)^{-\beta}\right]^{-a}\right\}^{-2} \\
& =(1-p) \frac{a \beta}{\alpha}\left(\frac{x}{\alpha}\right)^{\beta-1}\left[1+\left(\frac{x}{\alpha}\right)^{-\beta}\right]^{-(a-1)}\left[1+\left(\frac{x}{\alpha}\right)^{\beta}\right]^{-2} \times \\
& \sum_{j=0}^{\infty}(j+1) p^{j}\left[1+\left(\frac{x}{\alpha}\right)^{-\beta}\right]^{-a j} \\
& =\frac{a \beta}{\alpha}\left(\frac{x}{\alpha}\right)^{\beta-1}\left[1+\left(\frac{x}{\alpha}\right)^{-\beta}\right]^{-(a-1)}\left[1+\left(\frac{x}{\alpha}\right)^{\beta}\right]^{-2} \times \\
& \sum_{j=0}^{\infty}(j+1)(1-p) p^{j}\left[1+\left(\frac{x}{\alpha}\right)^{-\beta}\right]^{-a j} \text {. }
\end{aligned}
$$


Levando em consideração a função densidade da LLE dada por

$$
f_{\alpha, \beta, a}(x)=\frac{a \beta}{\alpha}\left(\frac{x}{\alpha}\right)^{\beta-1}\left[1+\left(\frac{x}{\alpha}\right)^{-\beta}\right]^{-(a-1)}\left[1+\left(\frac{x}{\alpha}\right)^{\beta}\right]^{-2},
$$

obtêm-se a expansão para a densidade da LLEGII, sendo uma combinação linear das distribuições log-logísticas exponenciadas, expressa por

$$
f_{\alpha, \beta, a, p}(x)=(1-p) f_{\alpha, \beta, a}(x) \sum_{j=0}^{\infty} w_{j}\left[F_{\alpha, \beta, a}(x)\right]^{j}
$$

em que os coeficientes $w_{j}=(j+1) p^{j}$ na expressão (31) são considerados como os pesos ponderados que multiplicam a família das distribuições log-logísticas exponenciadas.

\subsubsection{Momentos}

Como alternativa para o cálculo dos momentos pode ser escrita em termos dos momentos de probabilidade ponderada, que é uma generalização usual dos momentos ordinários de uma distribuição de probabilidade, Hosking et al. (1985), introduzidos por Greenwood et al. (1979). Nesta seção inicia-se com o teorema de momentos de probabilidade ponderada para a distribuição LLE, assim

Teorema 1. Se $X \sim \operatorname{LLE}(\alpha, \beta, a)$, para $\beta>r$, o $r$-ésimo momento de $X$ pode ser expressado como

$$
M(r, n)=a \alpha^{r} \mathrm{~B}\left(a(n+1)+\frac{r}{\beta}, 1-\frac{r}{\beta}\right)
$$

em que $\mathrm{B}(a, b)=\Gamma(a) \Gamma(b) / \Gamma(a+b)$ é a função beta.

\section{Prova:}

Os momentos de probabilidade ponderada de uma variável aleatória $X$ com função densidade, $f_{\alpha, \beta, a}(x)$ e a função de distribuição acumulada $F_{\alpha, \beta, a}(x)$ da distribuição LLE definida pelas equações (4) e (3), respectivamente é definido por $M(r, n)=E\left\{X^{r} F(x)^{n}\right\}$ para $r$ e $n$ inteiros positivos. Assim tem-se

$$
\begin{aligned}
M(r, n) & =E\left\{X^{r} F(x)^{n}\right\}=\int_{0}^{\infty} x^{r} f_{\alpha, \beta, a}(x) F_{\alpha, \beta, a}(x)^{n} d x \\
& =\int_{0}^{\infty} x^{r} \frac{a \beta}{\alpha}\left(\frac{x}{\alpha}\right)^{\beta-1}\left[1+\left(\frac{x}{\alpha}\right)^{-\beta}\right]^{-(a-1)}\left[1+\left(\frac{x}{\alpha}\right)^{\beta}\right]^{-2}\left\{\left[1+\left(\frac{x}{\alpha}\right)^{-\beta}\right]^{-a}\right\} d x \\
& =\frac{a \beta}{\alpha^{-\beta}} \int_{0}^{\infty} x^{r} x^{-\beta-1}\left[1+\left(\frac{x}{\alpha}\right)^{-\beta}\right]^{-a(n+1)-1} d x .
\end{aligned}
$$


Considerando a seguinte mudança de variável, $k=\left[1+\left(\frac{x}{\alpha}\right)^{-\beta}\right]^{-a(n+1)}$, substituindo na última integral, obtem-se

$$
M(r, n)=\frac{\alpha^{r}}{(n+1)} \int_{0}^{1}\left(k^{\frac{1}{a(n+1)}}\right)^{\frac{r}{\beta}}\left(1-k^{\frac{1}{a(n+1)}}\right)^{-\frac{r}{\beta}} d k
$$

reparametrizando $u=k^{\frac{1}{a(n+1)}}$ na integral, obtém-se a seguinte expressão

$$
M(r, n)=a \alpha^{r} \int_{0}^{1} u^{\frac{r}{\beta}+a(n+1)-1}(1-u)^{-\frac{r}{\beta}} d u .
$$

Finalmente, $M(r, n)$, pode ser escrito em termos da função beta.

$$
M(r, n)=a \alpha^{r} \mathrm{~B}\left(a(n+1)+\frac{r}{\beta}, 1-\frac{r}{\beta}\right) .
$$

Dessa última expressão, se $a=1$ obtém-se os momentos de probabilidade ponderada da distribuição log-logística, isto é

$$
M(r, n)=\alpha^{r} \mathrm{~B}\left(n+1+\frac{r}{\beta}, 1-\frac{r}{\beta}\right) .
$$

Se $a=1$ e $n=0$, obtém-se os momentos ordinários da distribuição log-logística dada por

$$
M(r, 0)=\mu_{r}^{\prime}=\alpha^{r} \mathrm{~B}\left(1+\frac{r}{\beta}, 1-\frac{r}{\beta}\right) .
$$

Os momentos das distribuições LLEGI e LLEGII também podem ser obtidos em termos das expansões das funções densidade dadas pelas expressões (26) e (31), respectivamente.

Teorema 2. Se $X \sim \operatorname{LLEGI}(\alpha, \beta, a, p)$, para $0<p<1$, então o $r$-ésimo momento de $X$, é dado por

$$
\mu_{r}^{\prime}=a \alpha^{r}(1-p) \sum_{j=0}^{\infty} \sum_{i=0}^{j} w_{j}(-1)^{i}\left(\begin{array}{l}
j \\
i
\end{array}\right) \mathrm{B}\left(a(i+1)+\frac{r}{\beta}, 1-\frac{r}{\beta}\right),
$$

em que $\mathrm{B}(.,$.$) é a função beta e w_{j}=(j+1) p^{j}$ é o coeficiente de ponderação.

\section{Prova:}

Para a distribuição LLEGI os momentos são obtidos resolvendo a equação

$$
\mu_{r}^{\prime}=\int_{0}^{\infty} x^{r} f(x) d x, \quad x>0
$$

considerando que $f(x)$ é a expansão da fdp da distribuição LLEGI dada por (26), então o $r$-ésimo momento é expressa por

$$
\begin{aligned}
\mu_{r}^{\prime} & =\int_{0}^{\infty} x^{r}(1-p) f_{\alpha, \beta, a}(x) \sum_{j=0}^{\infty} \sum_{i=0}^{j} w_{j}(-1)^{i}\left(\begin{array}{c}
j \\
i
\end{array}\right) F_{\alpha, \beta, a}(x)^{i} d x \\
& =(1-p) \sum_{j=0}^{\infty} \sum_{i=0}^{j} w_{j}(-1)^{i}\left(\begin{array}{c}
j \\
i
\end{array}\right) \int_{0}^{\infty} x^{r} f_{\alpha, \beta, a}(x) F_{\alpha, \beta, a}(x)^{i} d x
\end{aligned}
$$


A integral em (35) pode ser escrita em termos dos momentos de probabilidade ponderada da variável aleatória $X$ que segue uma distribuição LLE. Dessa forma aplicando o teorema (1), obtém-se

$$
\mu_{r}^{\prime}=a \alpha^{r}(1-p) \sum_{j=0}^{\infty} \sum_{i=0}^{j} w_{j}(-1)^{i}\left(\begin{array}{l}
j \\
i
\end{array}\right) \mathrm{B}\left(a(i+1)+\frac{r}{\beta}, 1-\frac{r}{\beta}\right) .
$$

Teorema 3. Se $X \sim \operatorname{LLEGII}(\alpha, \beta, a, p)$, para $0<p<1$, então o $r$-ésimo momento $X$ é dado por

$$
\mu_{r}^{\prime}=a \alpha^{r}(1-p) \sum_{j=0}^{\infty} w_{j} \mathrm{~B}\left(a(j+1)+\frac{r}{\beta}, 1-\frac{r}{\beta}\right),
$$

em que $\mathrm{B}(.,$.$) é a função beta e w_{j}=(j+1) p^{j}$ é o coeficiente de ponderação.

\section{Prova:}

Para a distribuição LLEGII, os momentos são obtidos resolvendo a equação (34), em que $f(x)$ é a expansão da fdp da distribuição LLEGII dada por (31), então o $r$-ésimo momento é expressa por

$$
\begin{aligned}
\mu_{r}^{\prime} & =\int_{0}^{\infty} x^{r}(1-p) f_{\alpha, \beta, a}(x) \sum_{j=0}^{\infty} w_{j} F_{\alpha, \beta, a}(x)^{j} d x \\
& =(1-p) \sum_{j=0}^{\infty} w_{j} \int_{0}^{\infty} x^{r} f_{\alpha, \beta, a}(x) F_{\alpha, \beta, a}(x)^{j} d x .
\end{aligned}
$$

A integral em (37) pode ser escrita em termos dos momentos de probabilidade ponderada da variável aleatória $X$ que segue uma distribuição LLE. Então aplicando o teorema (1), obtém-se

$$
\mu_{r}^{\prime}=a \alpha^{r}(1-p) \sum_{j=0}^{\infty} w_{j} \mathrm{~B}\left(a(j+1)+\frac{r}{\beta}, 1-\frac{r}{\beta}\right) .
$$

\subsubsection{Assimetria}

O coeficiente de assimetria é um indicador que permite estabelecer o grau de afastamento (ou assimetria) que uma distribuição apresenta de seu eixo de simetria (eixo de referência, traçado sobre o valor da média da distribuição). Este afastamento pode caracterizar as distribuições de frequência em: assimétrica à direita ou positiva, assimétrica à esquerda ou negativa e assimetria nula ou simétrica. Segundo Nadarajh e Kotz (2004), essa medida pode ser definida por,

$$
A(X)=\frac{E\left[X^{3}\right]-3 E[X] E\left[X^{2}\right]+2 E^{3}[X]}{\operatorname{Var}^{3 / 2}(X)}
$$

\subsubsection{Curtose}

O coeficiente de curtose é uma medida que estabelece o grau de achatamento da distribuição em relação à distribuição normal padrão. São três tipos de curvas da distribuição 
no que se refere à curtose: leptocúrtica, mesocúrtica e platicúrtica. Essa medida pode ser definido pela equação (39) segundo Nadarajh e Kotz (2004),

$$
K(X)=\frac{E\left[X^{4}\right]-4 E[X] E\left[X^{3}\right]+6 E\left[X^{2}\right] E^{2}[X]-3 E^{4}[X]}{\operatorname{Var}^{2}(X)} .
$$

(a)

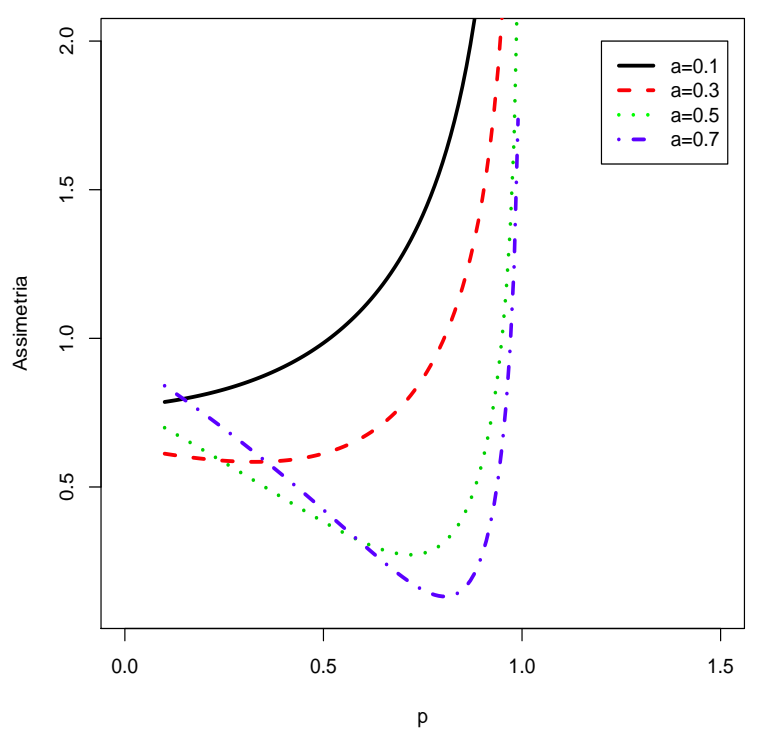

(b)

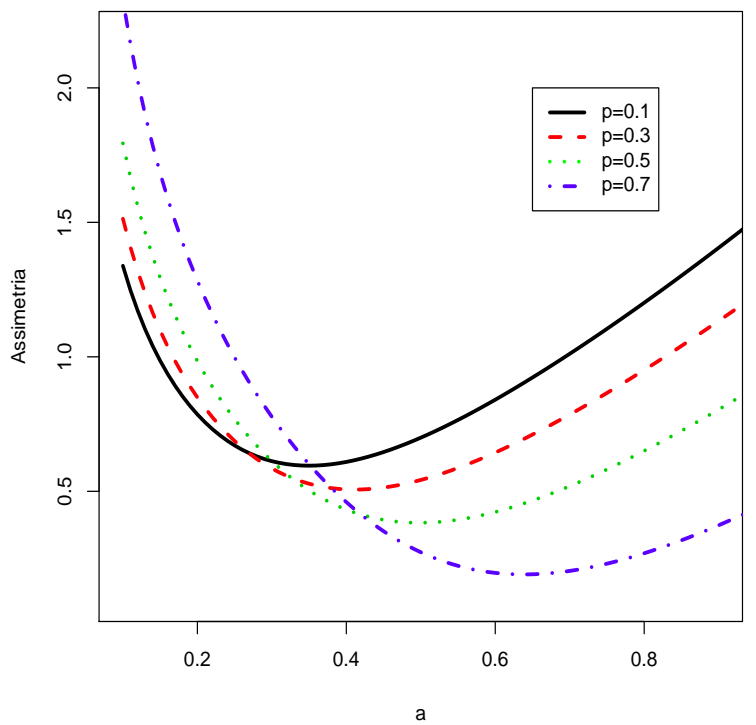

Figura 10 - Assimetria da distribuição LLEGI para alguns valores dos parâmetros. (a) Como função de $p$ para alguns valores de $a$ fixando $\alpha=1,5$ e $\beta=3,5$. (b) Como função de $a$ para alguns valores de $p$ fixando $\alpha=1,5$ e $\beta=3,5$

Portanto, as medidas da assimetria e curtose para as distribuições LLEGI e LLEGII, podem ser calculados a partir dos momentos ordinários, usando as relações descritas em (38) e (39).

Pode-se observar nas Figuras 10 e 11, os gráficos da assimetria das distribuições LLEGI e LLEGII, respectivamente, da mesma forma, observa-se as Figuras 12 e 13, os gráficos da curtose das distribuições, como função de $a$ para alguns valores de $p$ e como função de $p$ para alguns valores de $a$, considerando como fixo alguns valores dos parâmetros $\alpha$ e $\beta$. Esses gráficos revelam a existência de uma grande flexibilidade das curvas de assimetria e curtose.

\subsubsection{Função Geradora de Momentos}

Teorema 4. Se $X \sim \operatorname{LLEGI}(\alpha, \beta, a, p)$, para $\beta>r$ e $0<p<1$, então a função 
(a)

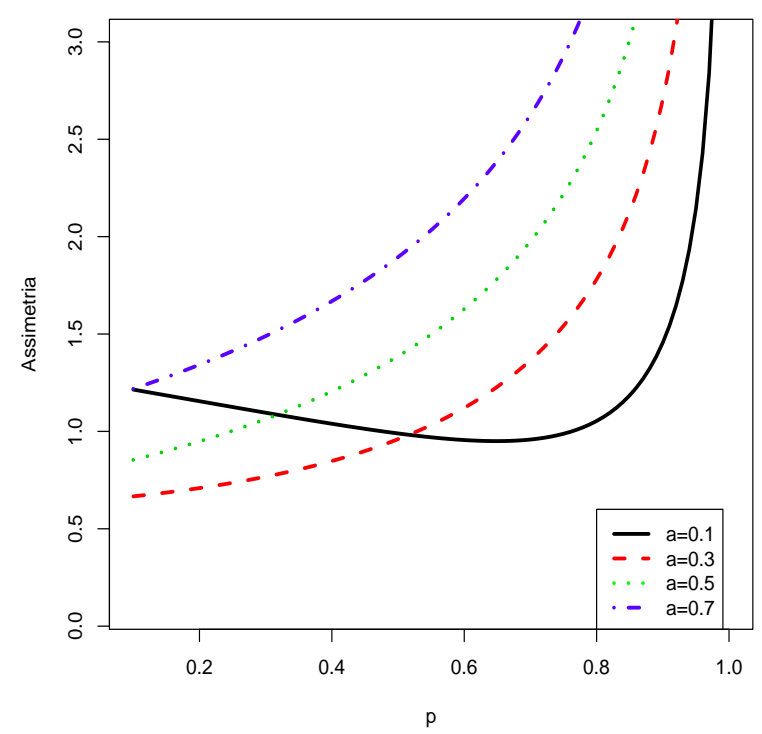

(b)

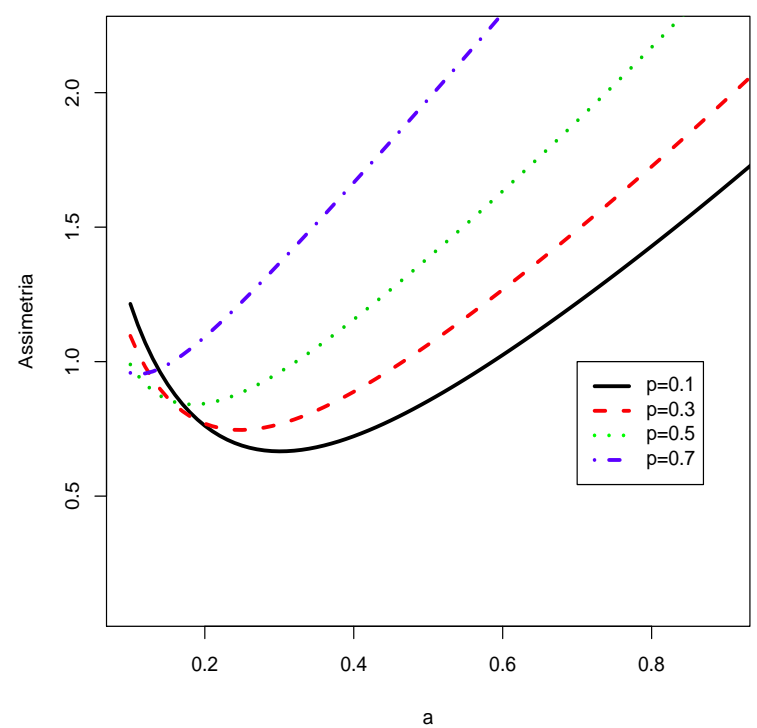

Figura 11 - Assimetria da distribuição LLEGII para alguns valores dos parâmetros. (a) Como função de $p$ para alguns valores de $a$ com $\alpha=1,5$ e $\beta=3,5$. (b) Como função de $a$ para alguns valores de $p$ fixando $\alpha=1,5$ e $\beta=3,0$

(a)

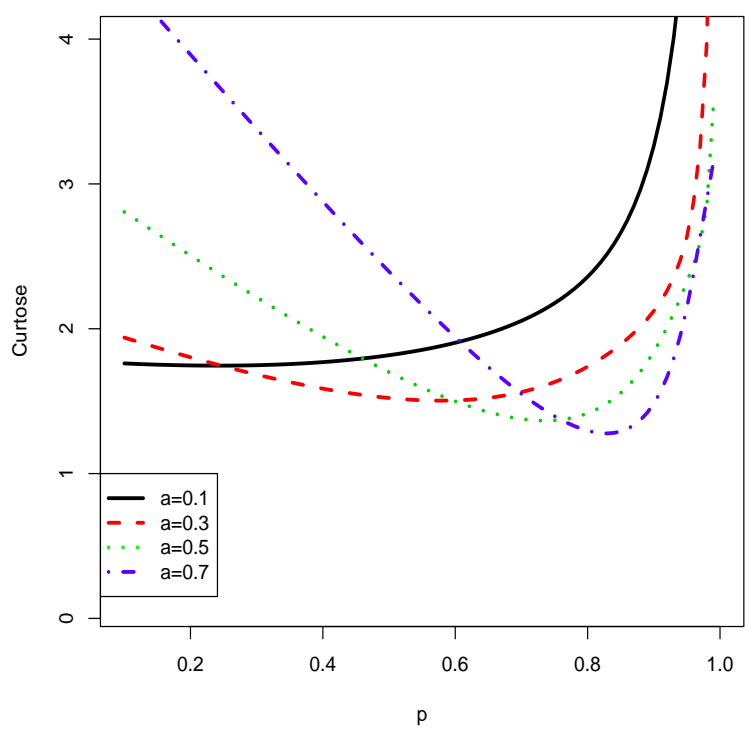

(b)

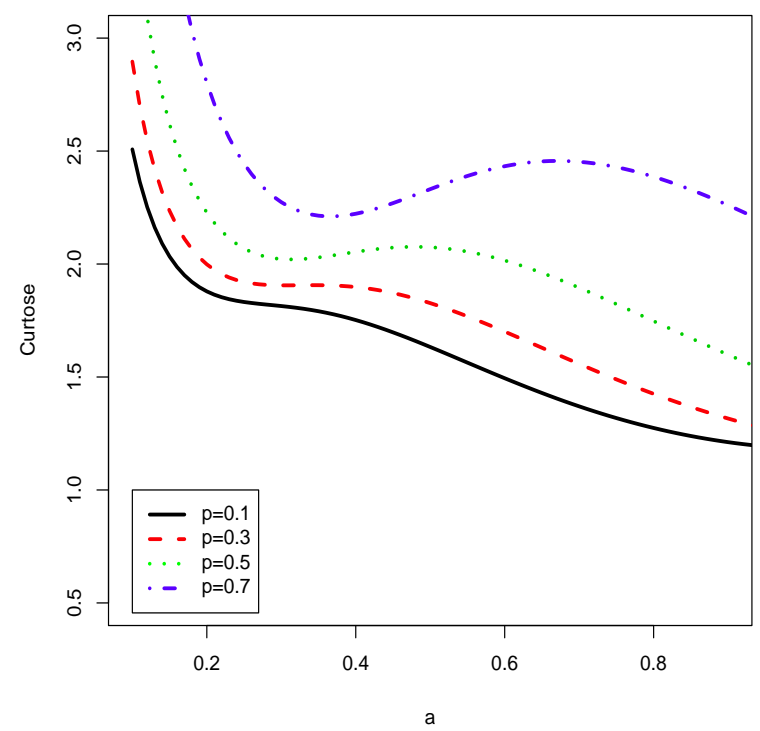

Figura 12 - Curtose da distribuição LLEGI para alguns valores dos parâmetros. (a) Como função de $p$ para alguns valores de $a$ fixando $\alpha=1,5$ e $\beta=4,5$. (b) Como função de $a$ para alguns valores de $p$ $\operatorname{com} \alpha=1,0$ e $\beta=5,5$ 
(a)

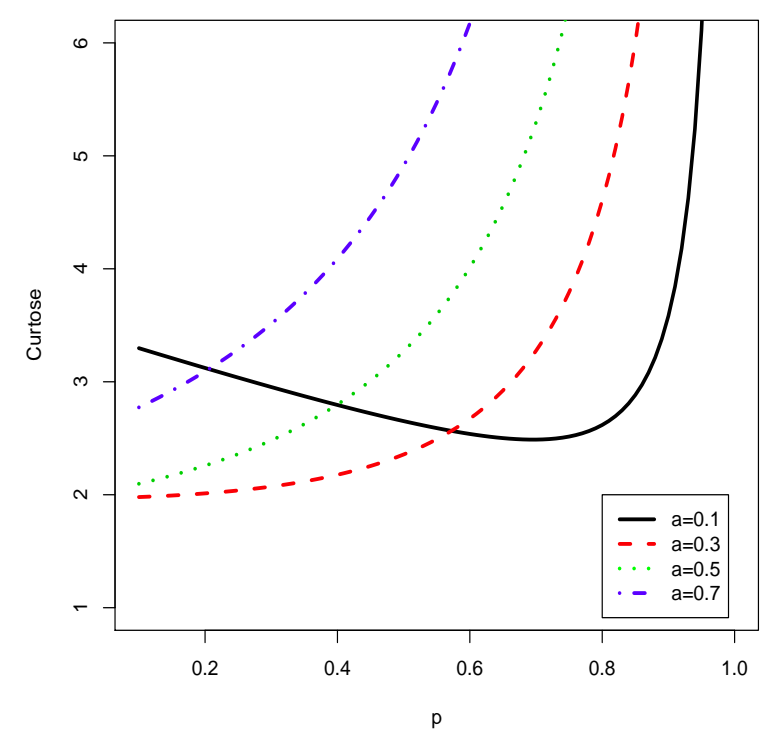

(b)

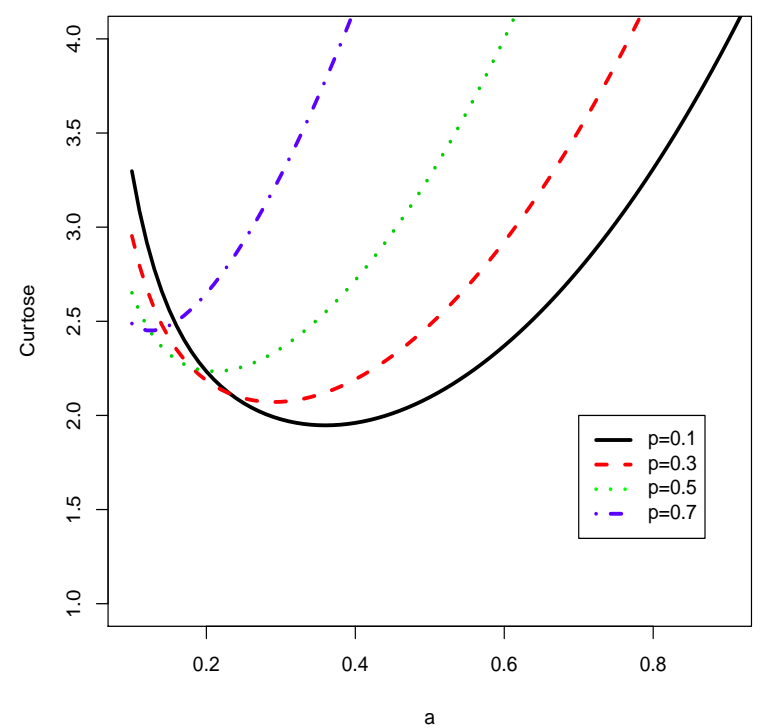

Figura 13 - Curtose da distribuição LLEGII para alguns valores dos parâmetros. (a) Como função de $p$ para alguns valores de $a \operatorname{com} \alpha=1,5$ e $\beta=3,0$. (b) Como função de $a$ para alguns valores de $p$ considerando $\alpha=1,5$ e $\beta=3,5$

geradora de momentos (fgm) é dada por

$$
M_{X}(t)=a(1-p) \sum_{j=0}^{\infty} \sum_{i=0}^{j} \sum_{r=0}^{\infty} \frac{t^{r}}{r !} \alpha^{r} w_{j}(-1)^{i}\left(\begin{array}{l}
j \\
i
\end{array}\right) \mathrm{B}\left(a(i+1)+\frac{r}{\beta}, 1-\frac{r}{\beta}\right),
$$

em que B $(.,$.$) é a função beta e w_{j}=(j+1) p^{j}$ é o coeficiente de ponderação.

\section{Prova:}

A fgm da distribuição LLEGI é calculado a partir da seguinte relação

$$
M_{X}(t)=\int_{0}^{\infty} e^{x t} f(x) d x
$$

considerando a expansão da função de densidade, $f(x)$, da distribuição LLEGI dada pela expressão (26), e utilizando a expansão em série de Maclaurin da função exponencial dada por

$$
e^{x t}=\sum_{r=0}^{\infty} \frac{(x t)^{r}}{r !}
$$

obtém-se

$$
\begin{aligned}
M_{X}(t) & =\int_{0}^{\infty} \sum_{r=0}^{\infty} \frac{(x t)^{r}}{r !} f(x) d x=\sum_{r=0}^{\infty} \frac{t^{r}}{r !} \int_{0}^{\infty} x^{r} f(x) d x \\
& =\sum_{r=0}^{\infty} \frac{t^{r}}{r !} \mu_{r}^{\prime} .
\end{aligned}
$$


A expressão (43) nos mostra, que se todos os momentos da variável aleatória $X$ são conhecidas, pode-se determinar a fgm de $X$ somando os termos da série nessa expressão.

A expressão $\mu_{r}^{\prime}$ em (40) pode ser escrita em termos dos momentos ordinários da variável aleatória $X$ que segue uma distribuição LLEGI; isto é, aplicando o teorema (2), obtém-se

$$
M_{X}(t)=a(1-p) \sum_{j=0}^{\infty} \sum_{i=0}^{j} \sum_{r=0}^{\infty} \frac{t^{r}}{r !} \alpha^{r} w_{j}(-1)^{i}\left(\begin{array}{l}
j \\
i
\end{array}\right) \mathrm{B}\left(a(i+1)+\frac{r}{\beta}, 1-\frac{r}{\beta}\right) .
$$

Teorema 5. Se $X \sim \operatorname{LLEGII}(\alpha, \beta, a, p)$, para $\beta>r$ e $0<p<1$, então a fgm é dada por

$$
M_{X}(t)=a(1-p) \sum_{r=0}^{\infty} \sum_{j=0}^{\infty} \frac{t^{r}}{r !} \alpha^{r} w_{j} \mathrm{~B}\left(a(j+1)+\frac{r}{\beta}, 1-\frac{r}{\beta}\right)
$$

em que B $(.,$.$) é a função beta e w_{j}=(j+1) p^{j}$ é o coeficiente de ponderação.

\section{Prova:}

A fgm da distribuição LLEGII é calculada a partir da relação (41), considerando a expansão da densidade, $f(x)$, da distribuição LLEGII dada pela expressão (31), e utilizando a expansão em série de Maclaurin da função exponencial de (42), obtém-se

$$
\begin{aligned}
M_{X}(t) & =\int_{0}^{\infty} \sum_{r=0}^{\infty} \frac{(x t)^{r}}{r !} f(x) d x=\sum_{r=0}^{\infty} \frac{t^{r}}{r !} \int_{0}^{\infty} x^{r} f(x) d x \\
& =\sum_{r=0}^{\infty} \frac{t^{r}}{r !} \mu_{r}^{\prime} .
\end{aligned}
$$

Substituindo $\mu_{r}^{\prime}$ na equação (45) em termos dos momentos ordinários da variável aleatória $X$ que segue uma distribuição LLEGII e aplicando o teorema (3), obtém-se

$$
M_{X}(t)=a(1-p) \sum_{r=0}^{\infty} \frac{t^{r}}{r !} \alpha^{r} \sum_{j=0}^{\infty} w_{j} \mathrm{~B}\left(a(j+1)+\frac{r}{\beta}, 1-\frac{r}{\beta}\right) .
$$

\subsubsection{Desvios médios}

A quantidade de dispersão em uma população pode ser medida pela totalidade de valores absolutos dos desvios em relação à média (no caso de uma distribuição simétrica) ou em relação à mediana (no caso de uma distribuição assimétrica).

Seja $X \sim \operatorname{LLEG}(\alpha, \beta, a, p)$ com média $\mu_{1}^{\prime}=E(X)$, e mediana $M$, então os desvios médios em relação à média e os desvios médios em relação à mediana são definidos, respectivamente por

$$
\delta_{1}=\int_{0}^{\infty}\left|x-\mu_{1}^{\prime}\right| f(x) d x=2 \mu_{1}^{\prime} F\left(\mu_{1}^{\prime}\right)-2 I\left(\mu_{1}^{\prime}\right)
$$


$\mathrm{e}$

$$
\delta_{2}=\int_{0}^{\infty}|x-M| f(x) d x=\mu_{1}^{\prime}-2 I(M) .
$$

Definindo a integral $I(s)=\int_{0}^{s} x f(x) d x$, estas medidas podem ser calculadas a partir de:

\section{- Distribuição LLEGI}

Se $X$ é uma variável aleatória com distribuição LLEGI com fdp, $f(x)$, expressa pela equação (26), então a integral $I(s)$ é dada por

$$
I(s)=\int_{0}^{s} x(1-p) f_{\alpha, \beta, a}(x) \sum_{j=0}^{\infty} \sum_{i=0}^{j} w_{j}(-1)^{i}\left(\begin{array}{l}
j \\
i
\end{array}\right) F_{\alpha, \beta, a}(x)^{i} d x
$$

considerando a fdp e fda da distribuição LLE, dadas por (4) e (3), respectivamente, a integral $I(s)$ reduz-se

$$
I(s)=(1-p) \sum_{j=0}^{\infty} \sum_{i=0}^{j} w_{j}(-1)^{i}\left(\begin{array}{l}
j \\
i
\end{array}\right) \frac{a \beta}{\alpha} \int_{0}^{s} x\left(\frac{x}{\alpha}\right)^{-\beta-1}\left[1+\left(\frac{x}{\alpha}\right)^{-\beta}\right]^{-a(i+1)-1} d x .
$$

Substituindo $k=\left[1+\left(\frac{x}{\alpha}\right)^{-\beta}\right]^{-a(i+1)}$ na última integral, pode-se escrever $I(s)=(1-p) \sum_{j=0}^{\infty} \sum_{i=0}^{j} w_{j}(-1)^{i}\left(\begin{array}{l}j \\ i\end{array}\right) \frac{\alpha}{(i+1)} \int_{0}^{\left[1+\left(\frac{s}{\alpha}\right)^{-\beta}\right]^{-a(i+1)}}\left(k^{\frac{1}{a(i+1)}}\right)^{\frac{1}{\beta}}\left(1-k^{\frac{1}{a(i+1)}}\right)^{-\frac{1}{\beta}} d k$.

Reparametrizando $u=k^{\frac{1}{a(i+1)}}$ na integral, obtém-se $I(s)$ em termos da função beta incompleta da seguinte forma

$$
I(s)=a(1-p) \alpha \sum_{j=0}^{\infty} \sum_{i=0}^{j} w_{j}(-1)^{i}\left(\begin{array}{l}
j \\
i
\end{array}\right) \mathrm{B}_{F_{\alpha, \beta}(s)}\left(a(i+1)+\frac{1}{\beta}, 1-\frac{1}{\beta}\right),
$$

em que $F_{\alpha, \beta}(s)$ é a fda da distribuição log-logística em termos de $s$.

\section{- Distribuição LLEGII}

Se $X$ é uma variável aleatória com distribuição LLEGII com fdp, $f(x)$, expressa pela equação (31), então a integral $I(s)$ é dada por

$$
I(s)=\int_{0}^{s} x(1-p) f_{\alpha, \beta, a}(x) \sum_{j=0}^{\infty} w_{j} F_{\alpha, \beta, a}(x)^{j} d x
$$

considerando a fdp e fda da distribuição LLE dadas por (4) e (3), respectivamente, a integral $I(s)$ reduz-se

$$
I(s)=(1-p) \sum_{j=0}^{\infty} w_{j} \frac{a \beta}{\alpha} \int_{0}^{s} x\left(\frac{x}{\alpha}\right)^{-\beta-1}\left[1+\left(\frac{x}{\alpha}\right)^{-\beta}\right]^{-a(j+1)-1} d x
$$


Substituindo $k=\left[1+\left(\frac{x}{\alpha}\right)^{-\beta}\right]^{-a(j+1)}$ na integral, pode-se escrever

$$
I(s)=(1-p) \sum_{j=0}^{\infty} w_{j} \frac{\alpha}{(j+1)} \int_{0}^{\left[1+\left(\frac{s}{\alpha}\right)^{-\beta}\right]^{-a(j+1)}}\left(k^{\frac{1}{a(j+1)}}\right)^{\frac{1}{\beta}}\left(1-k^{\frac{1}{a(j+1)}}\right)^{-\frac{1}{\beta}} d k .
$$

Reparametrizando $u=k^{\frac{1}{a(j+1)}}$ na integral, obtém-se $I(s)$ em termos da função beta incompleta da seguinte forma

$$
I(s)=a(1-p) \alpha \sum_{j=0}^{\infty} w_{j} \mathrm{~B}_{F_{\alpha, \beta}(s)}\left(a(j+1)+\frac{1}{\beta}, 1-\frac{1}{\beta}\right),
$$

em que $F_{\alpha, \beta}(s)$ é a fda da distribuição log-logística em termos de $s$.

Considerando estes resultados, tem-se

- Distribuição LLEGI

Seja $X \sim \operatorname{LLEGI}(\alpha, \beta, a, p)$ e considerando o resultado da equação (48) na equação (46), obtém-se os desvios médios em relação à média para a distribuição LLEGI que é expressa por

$$
\delta_{1}=2 \mu_{1}^{\prime} F\left(\mu_{1}^{\prime}\right)-2 a(1-p) \alpha \sum_{j=0}^{\infty} \sum_{i=0}^{j} w_{j}(-1)^{i}\left(\begin{array}{l}
j \\
i
\end{array}\right) \mathrm{B}_{F_{\alpha, \beta}\left(\mu_{1}^{\prime}\right)}\left(a(i+1)+\frac{1}{\beta}, 1-\frac{1}{\beta}\right) .
$$

Similarmente utilizando a expressão (47), os desvios médios em relação à mediana $\delta_{2}$, da distribuição LLEGI, pode ser escrito como

$$
\delta_{2}=\mu_{1}^{\prime}-2 a(1-p) \alpha \sum_{j=0}^{\infty} \sum_{i=0}^{j} w_{j}(-1)^{i}\left(\begin{array}{l}
j \\
i
\end{array}\right) \mathrm{B}_{F_{\alpha, \beta}(M)}\left(a(i+1)+\frac{1}{\beta}, 1-\frac{1}{\beta}\right),
$$

em que, $\mathrm{B}_{F_{\alpha, \beta}(.)}(.,$.$) , é a função beta incompleta, F_{\alpha, \beta}\left(\mu_{1}^{\prime}\right)$ e $F_{\alpha, \beta}(M)$, é a fda da distribuição LL em termos da média $\mu_{1}^{\prime}$ e da mediana $M$, respectivamente.

- Distribuição LLEGII

Seja $X \sim \operatorname{LLEGII}(\alpha, \beta, a, p)$ e considerando o resultado da equação (49) na equação (46), então os desvios médios em relação à média para a distribuição LLEGII é expressa por

$$
\delta_{1}=2 \mu_{1}^{\prime} F\left(\mu_{1}^{\prime}\right)-2 a(1-p) \alpha \sum_{j=0}^{\infty} w_{j} \mathrm{~B}_{F_{\alpha, \beta}\left(\mu_{1}^{\prime}\right)}\left(a(j+1)+\frac{1}{\beta}, 1-\frac{1}{\beta}\right) .
$$

Similarmente utilizando a expressão (47), os desvios médios em relação à mediana, $\delta_{2}$, da distribuição LLEGII, está expressa por

$$
\delta_{2}=\mu_{1}^{\prime}-2 a(1-p) \alpha \sum_{j=0}^{\infty} w_{j} \mathrm{~B}_{F_{\alpha, \beta}(M)}\left(a(j+1)+\frac{1}{\beta}, 1-\frac{1}{\beta}\right) .
$$


Como no caso da distribuiçã LLEGI, em que $\mathrm{B}_{F_{\alpha, \beta}(.)}(.,$.$) , é a função beta incompleta,$ $F_{\alpha, \beta}\left(\mu_{1}^{\prime}\right)$ e $F_{\alpha, \beta}(M)$ é a fda da distribuição log-logística em termos da média $\mu_{1}^{\prime}$ e da mediana $M$.

A Curva de Lorenz (ou curva de concentração de Lorenz) consiste num gráfico muito utilizado pelos economistas e que procura ilustrar a desigualdade existente na distribuição do rendimento entre as famílias numa determinada economia ou sociedade.

\subsubsection{Curvas de Bonferroni e Lorenz}

As curvas de Bonferroni e Lorenz são gráficos utilizados na área de econômia para o estudo da renda e da pobreza de uma população e que procura ilustrar a desigualdade existente na distribuição do rendimento entre as famílias numa determinada economia ou sociedade, também são aplicados em outras áreas, como demografia, confiabilidade, seguros, medicina e dentre outros. As expressões das curvas são definidas por

$$
B(v)=\frac{1}{v \mu_{1}^{\prime}} \int_{0}^{q} x f(x) d x
$$

$\mathrm{e}$

$$
L(v)=\frac{1}{\mu_{1}^{\prime}} \int_{0}^{q} x f(x) d x,
$$

em que $\mu_{1}^{\prime}=E(X)$ e $q=F^{-1}(v)$ é a inversa da fda da distribuição da LLEGI ou LLEGII segundo corresponda.

\section{- Distribuição LLEGI}

A inversa da fda da distribuição da LLEGI para q, dada na seção (3.3.1) para um dado valor $v$, considerando a expressão (48) na expressão (50), obtém-se

$$
B(v)=\frac{1}{v \mu_{1}^{\prime}} a(1-p) \alpha \sum_{j=0}^{\infty} \sum_{i=0}^{j} w_{j}(-1)^{i}\left(\begin{array}{l}
j \\
i
\end{array}\right) \mathrm{B}_{G(q)}\left(a(i+1)+\frac{1}{\beta}, 1-\frac{1}{\beta}\right) .
$$

A Curva de Lorenz da expressão (51) para a distribuição LLEGI, pode ser representada por

$$
L(v)=\frac{1}{\mu_{1}^{\prime}} a(1-p) \alpha \sum_{j=0}^{\infty} \sum_{i=0}^{j} w_{j}(-1)^{i}\left(\begin{array}{l}
j \\
i
\end{array}\right) \mathrm{B}_{G(q)}\left(a(i+1)+\frac{1}{\beta}, 1-\frac{1}{\beta}\right),
$$

em que $G(q)$ tem a forma da fda da distribuição log-logística.

\section{- Distribuição LLEGII}

Considerando a inversa da fda da distribuição da LLEGII para $q$, dada na seção (3.3.2) 
para um dado valor $v$, e considerando (49) na expressão (50), obtém-se

$$
B(v)=\frac{1}{v \mu_{1}^{\prime}} a(1-p) \alpha \sum_{j=0}^{\infty} w_{j} \mathrm{~B}_{G(q)}\left(a(j+1)+\frac{1}{\beta}, 1-\frac{1}{\beta}\right) .
$$

De forma similar, a Curva de Lorenz da expressão (51) para a distribuição LLEGII, está representada por

$$
L(v)=\frac{1}{\mu_{1}^{\prime}} a(1-p) \alpha \sum_{j=0}^{\infty} w_{j} \mathrm{~B}_{G(q)}\left(a(j+1)+\frac{1}{\beta}, 1-\frac{1}{\beta}\right),
$$

em que $G(q)$ tem a forma da fda da distribuição log-logística.

\subsection{Estimação por Máxima Verossimilhança}

A estimação dos parâmetros das distribuições LLEGI e LLEGII são feitas utilizando o método de máxima verossimilhança para amostras completas. Seja $x_{1}, \ldots, x_{n}$ uma amostra aleatória de tamanho $n$, em que cada $x_{i}, i=1, \ldots, n$, possui uma distribuição $\operatorname{LLEGI}(\alpha, \beta, a, p)$ ou $\operatorname{LLEGII}(\alpha, \beta, a, p)$ com vetor de parâmetros $\boldsymbol{\theta}=(\alpha, \beta, a, p)^{T}$. O logaritmo da função de verossimilhança para o vetor de parâmetros $\boldsymbol{\theta}$, é dado por

- Distribuição LLEGI

$$
\begin{aligned}
l(\theta)= & n \log \left[\frac{(1-p) a \beta}{\alpha}\right]-(\beta+1) \sum_{i=1}^{n} \log \left(\frac{x_{i}}{\alpha}\right)-(a+1) \sum_{i=1}^{n} \log \left[1+\left(\frac{x_{i}}{\alpha}\right)^{-\beta}\right]- \\
& 2 \sum_{i=1}^{n} \log \left\{1-p\left[1-\left[1+\left(\frac{x_{i}}{\alpha}\right)^{-\beta}\right]^{-a}\right]\right\} .
\end{aligned}
$$

- Distribuição LLEGII

$$
\begin{aligned}
l(\theta)= & n \log \left[\frac{(1-p) a \beta}{\alpha}\right]-(\beta+1) \sum_{i=1}^{n} \log \left(\frac{x_{i}}{\alpha}\right)-(a+1) \sum_{i=1}^{n} \log \left[1+\left(\frac{x_{i}}{\alpha}\right)^{-\beta}\right]- \\
& 2 \sum_{i=1}^{n} \log \left\{1-p\left[1+\left(\frac{x_{i}}{\alpha}\right)^{-\beta}\right]^{-a}\right\} .
\end{aligned}
$$

Os componentes do vetor escore $\boldsymbol{U}=\left(\frac{\partial l(\boldsymbol{\theta})}{\partial \alpha}, \frac{\partial l(\boldsymbol{\theta})}{\partial \beta}, \frac{\partial l(\boldsymbol{\theta})}{\partial a}, \frac{\partial l(\boldsymbol{\theta})}{\partial p}\right)^{T}$ são obtidos por diferenciação; isto é 
- Distribuição LLEGI

$$
\begin{aligned}
& \frac{\partial l(\boldsymbol{\theta})}{\partial \alpha}=\frac{n \beta}{\alpha}-\frac{(a+1) \beta}{\alpha^{-\beta+1}} \sum_{i=1}^{n} \frac{x_{i}^{-\beta}}{\left[1+\left(\frac{x_{i}}{\alpha}\right)^{-\beta}\right]}+\frac{2 a \beta p}{\alpha^{-\beta+1}} \sum_{i=1}^{n} \frac{x_{i}^{-\beta}\left[1+\left(\frac{x_{i}}{\alpha}\right)^{-\beta}\right]^{-a-1}}{\left\{1-p\left[1-\left[1+\left(\frac{x_{i}}{\alpha}\right)^{-\beta}\right]^{-a}\right]\right\}}, \\
& \frac{\partial l(\boldsymbol{\theta})}{\partial \beta}=\frac{n}{\beta}-\sum_{i=1}^{n} \log \left(\frac{x_{i}}{\alpha}\right)+\frac{(a+1)}{\alpha^{-\beta}} \sum_{i=1}^{n} \frac{x_{i}^{-\beta} \ln \left(\frac{x_{i}}{\alpha}\right)}{\left[1+\left(\frac{x_{i}}{\alpha}\right)^{-\beta}\right]}- \\
& \frac{2 a p}{\alpha^{-\beta}} \sum_{i=1}^{n} \frac{x_{i}^{-\beta}\left[1+\left(\frac{x_{i}}{\alpha}\right)^{-\beta}\right]^{-a-1} \ln \left(\frac{x_{i}}{\alpha}\right)}{\left\{1-p\left[1-\left[1+\left(\frac{x_{i}}{\alpha}\right)^{-\beta}\right]^{-a}\right]\right\}} \\
& \frac{\partial l(\boldsymbol{\theta})}{\partial a}=\frac{n}{a}-\sum_{i=1}^{n} \log \left[1+\left(\frac{x_{i}}{\alpha}\right)^{-\beta}\right]+2 p \sum_{i=1}^{n} \frac{\left[1+\left(\frac{x_{i}}{\alpha}\right)^{-\beta}\right]^{-a} \ln \left[1+\left(\frac{x_{i}}{\alpha}\right)^{-\beta}\right]}{\left\{1-p\left[1-\left[1+\left(\frac{x_{i}}{\alpha}\right)^{-\beta}\right]^{-a}\right]\right\}} \\
& \frac{\partial l(\boldsymbol{\theta})}{\partial p}=-\frac{n}{(1-p)}-2 \sum_{i=1}^{n} \frac{-1+\left[1+\left(\frac{x_{i}}{\alpha}\right)^{-\beta}\right]^{-a}}{\left\{1-p\left[1-\left[1+\left(\frac{x_{i}}{\alpha}\right)^{-\beta}\right]^{-a}\right]\right\}} .
\end{aligned}
$$

- Distribuição LLEGII

$$
\begin{aligned}
\frac{\partial l(\boldsymbol{\theta})}{\partial \alpha}= & \frac{n \beta}{\alpha}-\frac{(a+1) \beta}{\alpha^{-\beta+1}} \sum_{i=1}^{n} \frac{x_{i}^{-\beta}}{\left[1+\left(\frac{x_{i}}{\alpha}\right)^{-\beta}\right]}-\frac{2 a \beta p}{\alpha^{-\beta+1}} \sum_{i=1}^{n} \frac{x_{i}^{-\beta}\left[1+\left(\frac{x_{i}}{\alpha}\right)^{-\beta}\right]^{-a-1}}{\left\{1-p\left[1+\left(\frac{x_{i}}{\alpha}\right)^{-\beta}\right]^{-a}\right\}}, \\
\frac{\partial l(\boldsymbol{\theta})}{\partial \beta}= & \frac{n}{\beta}-\sum_{i=1}^{n} \log \left(\frac{x_{i}}{\alpha}\right)+\frac{(a+1)}{\alpha^{-\beta}} \sum_{i=1}^{n} \frac{x_{i}^{-\beta} \ln \left(\frac{x_{i}}{\alpha}\right)}{\left[1+\left(\frac{x_{i}}{\alpha}\right)^{-\beta}\right]}+ \\
& \frac{2 a p}{\alpha^{-\beta}} \sum_{i=1}^{n} \frac{x_{i}^{-\beta}\left[1+\left(\frac{x_{i}}{\alpha}\right)^{-\beta}\right]^{-a-1} \ln \left(\frac{x_{i}}{\alpha}\right)}{\left\{1-p\left[1+\left(\frac{x_{i}}{\alpha}\right)^{-\beta}\right]^{-a}\right\}}, \\
\frac{\partial l(\boldsymbol{\theta})}{\partial a}= & \frac{n}{a}-\sum_{i=1}^{n} \log \left[1+\left(\frac{x_{i}}{\alpha}\right)^{-\beta}\right]-2 p \sum_{i=1}^{n} \frac{\left[1+\left(\frac{x_{i}}{\alpha}\right)^{-\beta}\right]^{-a} \ln \left[1+\left(\frac{x_{i}}{\alpha}\right)^{-\beta}\right]}{\left.\left\{1-\left(\frac{x_{i}}{\alpha}\right)^{-\beta}\right]^{-a}\right\}} \\
\frac{\partial l(\boldsymbol{\theta})}{\partial p}= & -\frac{n}{(1-p)}+2 \sum_{i=1}^{n} \frac{\left[1+\left(\frac{x_{i}}{\alpha}\right)^{-\beta}\right]^{-a}}{\left\{1-p\left[1+\left(\frac{x_{i}}{\alpha}\right)^{-\beta}\right]^{-a}\right\}}
\end{aligned}
$$

Consequentemente, o estimador de máxima verossimilhança (EMV) $\hat{\boldsymbol{\theta}}$ de $\boldsymbol{\theta}$ é obtido numéricamente a partir das equações não lineares

$$
\frac{\partial l(\boldsymbol{\theta})}{\partial \alpha}=\frac{\partial l(\boldsymbol{\theta})}{\partial \beta}=\frac{\partial l(\boldsymbol{\theta})}{\partial a}=\frac{\partial l(\boldsymbol{\theta})}{\partial p}=0 .
$$


Porém, para obter o EMV para os parâmetros $\alpha, \beta, a$ e $p$ é necessário a utilização de métodos iterativos. As estimativas podem ser obtidos numericamente, maximizando o logaritmo da função de verossimilhança e usando um algoritmo de otimização não linear, por exemplo, Newton-Raphson, o algoritmo Quase-Newton, algoritmo EM, etc. Para estimação intervalar e testes de hipóteses dos parâmetros do modelo, necessita-se da matriz $4 X 4$ de informação observada $\boldsymbol{J}=\boldsymbol{J}(\boldsymbol{\theta})$, assim

$$
\boldsymbol{J}(\boldsymbol{\theta})=-\left(\begin{array}{cccc}
J_{\alpha \alpha} & J_{\alpha \beta} & J_{\alpha a} & J_{\alpha p} \\
J_{\beta \alpha} & J_{\beta \beta} & J_{\beta a} & J_{\beta p} \\
J_{a \alpha} & J_{a \beta} & J_{a a} & J_{a p} \\
J_{p \alpha} & J_{p \beta} & J_{p a} & J_{p p}
\end{array}\right),
$$

em que os elementos da matriz $\boldsymbol{J}=\boldsymbol{J}(\boldsymbol{\theta})$ são dados no Apêndice A. Sob certas condições de regularidade que são satisfeitas para os parâmetros no interior do espaço paramétrico, mas não sobre a fronteira, a distribuição assintótica dos EMV é dada por $\sqrt{n}(\hat{\boldsymbol{\theta}}-\boldsymbol{\theta}) \sim \mathrm{N}_{4}\left(0, \boldsymbol{I}(\boldsymbol{\theta})^{-1}\right)$, em que $\boldsymbol{I}(\boldsymbol{\theta})$ é a matriz de informação esperada; isto é, $\boldsymbol{I}(\boldsymbol{\theta})=E[\boldsymbol{J}(\boldsymbol{\theta})]$ tal que $\boldsymbol{J}(\boldsymbol{\theta})=-\left\{\frac{\partial^{2} l(\boldsymbol{\theta})}{\partial \boldsymbol{\theta} \partial \boldsymbol{\theta}^{T}}\right\}$. As vezes não è fácil obter $\boldsymbol{I}(\boldsymbol{\theta})$, nesse caso uma boa aproximação é substituir por $\boldsymbol{J}(\hat{\boldsymbol{\theta}})$; isto é, a matriz de informação observada estimada em $(\hat{\boldsymbol{\theta}})$. A distribuição normal multivariada $\mathrm{N}_{4}\left(0, \boldsymbol{J}(\boldsymbol{\theta})^{-1}\right)$ pode ser utilizada na construção de intervalos de confiança aproximados para os parâmetros individuais. Um intervalo de confiança assintótico, com coeficiente de confiança $\gamma$ para cada parâmetro $\theta_{r}$ é obtido por meio da expressão

$$
A C I\left(\theta_{r}, 100(1-\gamma) \%\right)=\left(\hat{\theta}_{r}-z_{\gamma / 2} \sqrt{\hat{J}^{\theta_{r}, \theta_{r}}} \quad, \hat{\theta}_{r}+z_{\gamma / 2} \sqrt{\hat{J}^{\theta_{r}, \theta_{r}}}\right)
$$

em que, $\hat{J}^{\theta_{r}, \theta_{r}}$ é o $r$-ésimo elemento diagonal estimado de $\boldsymbol{J}(\hat{\boldsymbol{\theta}})^{-1}$ para $r=1,2,3,4$ e $z_{\frac{\gamma}{2}}$ é o quantil $1-\frac{\gamma}{2}$ da distribuição normal padrão.

Dentre os principais critérios de seleção de modelos utilizados em programas computacionais estão o critério de informação de Akaike - AIC (AKAIKE, 1974), o critério de informação bayesiano - BIC (SCHWARZ,1978) e o critério de informação de Akaike corrigido - CAIC (BOZDOGAN, 1987) os quais são baseados no valor do logaritmo de função de verossimilhança do modelo e dependem do número de parâmetros $q$ e são dados por

$$
\begin{aligned}
A I C & =-2 l(\boldsymbol{\theta})+2 q \\
B I C & =-2 l(\boldsymbol{\theta})+q \log (n) \\
C A I C & =A I C+\frac{2 q(q+1)}{n-q-1}
\end{aligned}
$$

valores menores de AIC, BIC e CAIC indicam modelos mais apropriados. 


\subsubsection{Testes de hipóteses}

Para testar a adequabilidade de um modelo, as hipóteses de interesse são da seguinte forma

$$
H o: \boldsymbol{\theta}=\boldsymbol{\theta}_{o} \quad \text { versus } H_{1}: \boldsymbol{\theta} \neq \boldsymbol{\theta}_{o}
$$

em que $\boldsymbol{\theta}_{o}$ é o vetor de parâmetros a ser testado. O teste de hipótese é feito utilizando a estatística da razão da verossimilhança (RV). O teste de razão de verossimilhança consiste em comparar os valores do logaritmo da função de verossimilhança do modelo geral e do modelo reduzido sob a hipótese nula Ho. A partir desses valores, a estatística da razão de verossimilhança é calculada por

$$
w=2[l(\hat{\alpha}, \hat{\beta}, \hat{a}, \hat{p})-l(\tilde{\alpha}, \tilde{\beta}, \tilde{a}, \tilde{p})]
$$

em que $l(\hat{\alpha}, \hat{\beta}, \hat{a}, \hat{p})$ é o logaritmo da função de verossimilhança do modelo geral e $l(\tilde{\alpha}, \tilde{\beta}, \tilde{a}, \tilde{p})$ denota o logaritmo da função de verossimilhança do modelo reduzido calculada sob Ho. A estatística $w$ sob Ho tem aproximadamente uma distribuição qui-quadrado com $k$ graus de liberdade, que corresponde a diferença do número de parâmetros dos modelos a serem comparados. Rejeita-se a hipótese nula $(H o)$ a um nível de $100 \alpha \%$ de significância, quando $w>\chi_{1-\alpha}^{2}(k)$ é o quantil $(1-\alpha)$ da distribuição qui-quadrado com $k$ graus de liberdade.

Por exemplo, para verificar a adequabilidade dos modelos usando as distribuições log-logística exponenciada e log-logística exponenciada geométrica tipo I para um determinado conjunto de dados, testar a hipótese da expressão (54) é equivalente a testar a hipótese do tipo $H o: p=0$ versus $H_{1}: H_{o}$ não é verdade, para o qual a estatística da razão de verossimilhança reduz-se a

$$
w=2[l(\hat{\alpha}, \hat{\beta}, \hat{a}, \hat{p})-l(\tilde{\alpha}, \tilde{\beta}, \tilde{a}, 0)]
$$

em que $\hat{\alpha}, \hat{\beta}, \hat{a}$ e $\hat{p}$ são os estimadores de máxima verossimilhança do modelo geral que segue da maximização do logaritmo da função de verossimilhança da expressão (52) e $\tilde{\alpha}, \tilde{\beta}$ e $\tilde{a}$ são os estimadores de máxima verossimilhança do modelo reduzido calculada sob $H_{o}$, ou seja, com $p=0$. 


\section{DISTRIBUIÇÃO LOGÍSTICA EXPONENCIADA GEOMÉTRICA}

\subsection{Distribuição logística exponenciada}

Assim como acontece com as distribuições Weibull, normal e log-logística, muitas vezes é conveniente trabalhar com o logaritmo dos tempos observados. Desta forma, se $X$ é uma variável aleatória que segue uma distribuição log-logística exponenciada com parâmetros $\alpha>0, \beta>0$ e $a>0$ então, seu logaritmo $Y=\log (X)$ segue uma distribuição logística exponenciada com fdp dada por

$$
g(y)=\frac{a}{\sigma} \exp \left(\frac{y-\mu}{\sigma}\right)\left[1+\exp \left(-\frac{y-\mu}{\sigma}\right)\right]^{-(a-1)}\left[1+\exp \left(\frac{y-\mu}{\sigma}\right)\right]^{-2}, \quad-\infty<y<\infty,
$$

em que, $-\infty<\mu<\infty$ é o parâmetro de locação e $\sigma>0$ é o parâmetro de escala, com reparametrização $\alpha=\exp (\mu)$ e $\beta=1 / \sigma$. Se $Y$ segue uma distribuição logística exponenciada com três parâmetros, denotada por $Y \sim \operatorname{LE}(\mu, \sigma, a)$. A fda para a variável aleatória $Y$ tem a seguinte forma

$$
G(y)=\left[1+\exp \left(-\frac{y-\mu}{\sigma}\right)\right]^{-a}
$$

consequentemente, a função de sobrevivência é dada por

$$
S(y)=1-\left[1+\exp \left(-\frac{y-\mu}{\sigma}\right)\right]^{-a},
$$

para simular dados de uma distribuição LE, utilizou-se a inversa da fda correspondente

$$
Q(u)=G^{-1}(u)=\mu-\sigma \log \left[\frac{1-u^{1 / a}}{u^{1 / a}}\right],
$$

em que $u$ tem distribuição uniforme $(0,1)$.

\subsection{Distribuição logística exponenciada geométrica com dupla ativação}

Seja $X$ uma variável aleatória contínua com fdp (11) ou (18) da distribuição LLEGI ou LLEGII, respectivamente. Considerando que a nova variável aleatória $Y=\log (X)$, segue uma distribuição reparametrizada em termos de $\alpha=\exp (\mu)$ e $\beta=\frac{1}{\sigma}$, denominada distribuição logística exponenciada geométrica tipo I (LEGI) ou distribuição logística exponenciada geométrica tipo II (LEGII), segundo corresponda. A nova função densidade, reduz-se

- Distribuição LEGI

$$
\begin{aligned}
f(y ; \mu, \sigma, a, p)= & \frac{(1-p) a}{\sigma} \exp \left(\frac{y-\mu}{\sigma}\right)\left[1+\exp \left(-\frac{y-\mu}{\sigma}\right)\right]^{-(a-1)} \times \\
& {\left[1+\exp \left(\frac{y-\mu}{\sigma}\right)\right]^{-2}\left\{1-p\left[1-\left[1+\exp \left(-\frac{y-\mu}{\sigma}\right)\right]^{-a}\right]\right\}^{-2}(56) }
\end{aligned}
$$


- Distribuição LEGII

$$
\begin{aligned}
f(y ; \mu, \sigma, a, p)= & \frac{(1-p) a}{\sigma} \exp \left(\frac{y-\mu}{\sigma}\right)\left[1+\exp \left(-\frac{y-\mu}{\sigma}\right)\right]^{-(a-1)} \times \\
& {\left[1+\exp \left(\frac{y-\mu}{\sigma}\right)\right]^{-2} \times\left\{1-p\left[1+\exp \left(-\frac{y-\mu}{\sigma}\right)\right]^{-a}\right\}^{-2}, }
\end{aligned}
$$

em que $-\infty<y<\infty,-\infty<\mu<\infty, \sigma>0, a>0$ e $p \in(0,1)$

Se $X$ é uma variável aleatória com função densidade (56) ou (57), pode-se escrever $Y \sim \operatorname{LEGI}(\mu, \sigma, a, p)$ ou $Y \sim \operatorname{LEGII}(\mu, \sigma, a, p)$, respectivamente.

As distribuições LEGI e LEGII possuem propriedades importantes como sub modelos de distribuições conhecidas, assim quando $p=0$, simplifica-se à distribuição logística exponenciada. Se $a=1$ e $p=0$, reduz-se à distribuição logística. A fdp das distribuições LEGI e LEGII são apresentados nas Figuras 14 e 15, para alguns valores fixados dos parâmetros $\mu$ e $\sigma$, variando os valores dos parâmetros $a$ e $p$, estes gráficos mostram grande flexibilidade para os diferentes valores dos parâmetros de forma $a$ e $\beta$.

A função de distribuição acumulada correspondente a (56) e (57) é expressa por

- Distribuição LEGI

$$
F(y ; \mu, \sigma, a, p)=\left[1+\exp \left(-\frac{y-\mu}{\sigma}\right)\right]^{-a}\left\{1-p\left[1-\left[1+\exp \left(-\frac{y-\mu}{\sigma}\right)\right]^{-a}\right]\right\}^{-1} .
$$

- Distribuição LEGII

$$
F(y ; \mu, \sigma, a, p)=(1-p)\left[1+\exp \left(-\frac{y-\mu}{\sigma}\right)\right]^{-a}\left\{1-p\left[1+\exp \left(-\frac{y-\mu}{\sigma}\right)\right]^{-a}\right\}^{-1} .
$$

Consequêntemente, a função de sobrevivência é dada por

- Distribuição LEGI

$$
S(y)=1-\left[1+\exp \left(-\frac{y-\mu}{\sigma}\right)\right]^{-a}\left\{1-p\left[1-\left[1+\exp \left(-\frac{y-\mu}{\sigma}\right)\right]^{-a}\right]\right\}^{-1} .
$$

- Distribuição LEGII

$$
S(y)=1-(1-p)\left[1+\exp \left(-\frac{y-\mu}{\sigma}\right)\right]^{-a}\left\{1-p\left[1+\exp \left(-\frac{y-\mu}{\sigma}\right)\right]^{-a}\right\}^{-1} .
$$


(a)

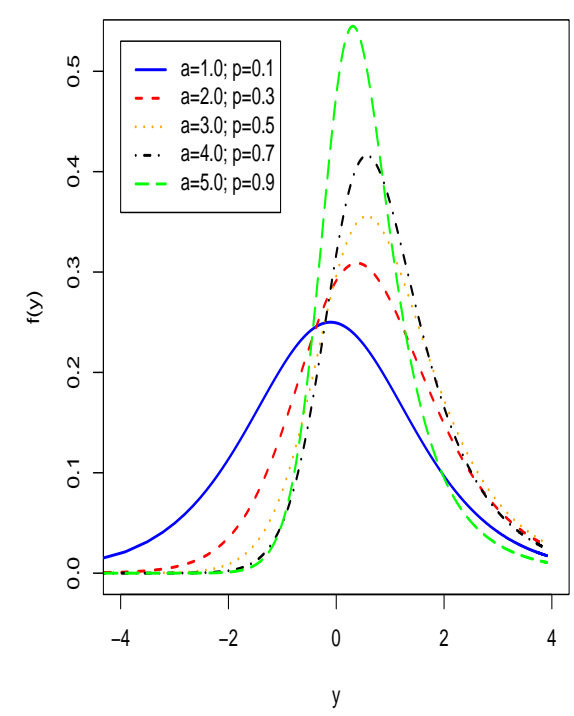

(b)

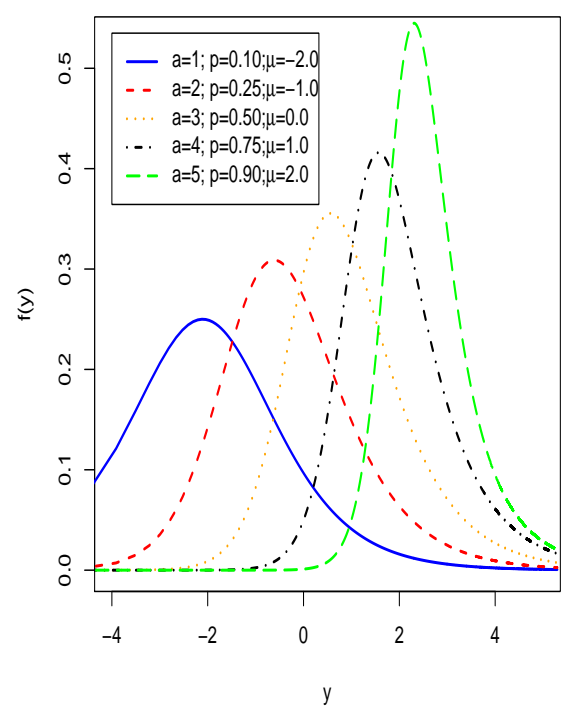

(c)

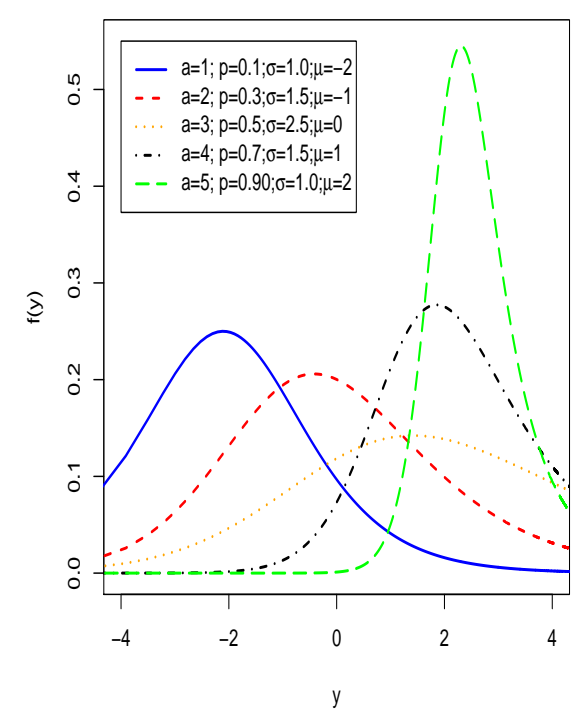

Figura 14 - Gráficos da fdp da distribuição LEGI, para alguns valores dos parâmetros. (a) Para diferentes valores de $a$ e $p$, com $\mu=0$ e $\sigma=1$. (b) Para diferentes valores de $a, p$ e $\mu$, com $\sigma=1$. (c) Para diferentes valores de $a, p, \mu$ e $\sigma$

\subsubsection{Expansão da função densidade da distribuição logística exponenciada geo- métrica}

- Distribuição LEGI

Pode-se escrever a fdp da distribuição da LEGI dada pela expressão (56), como uma 
(a)

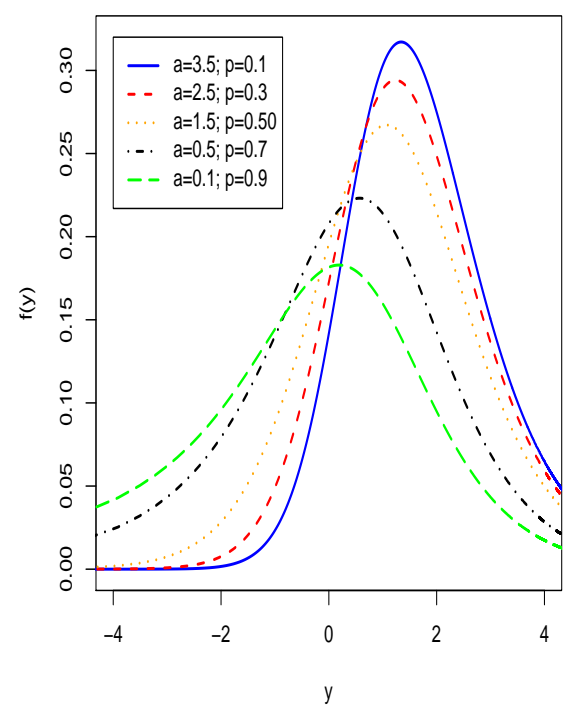

(b)

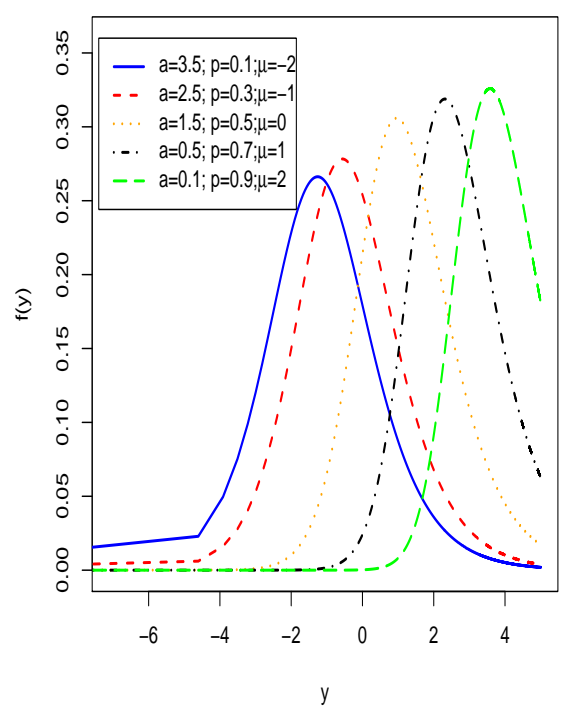

(c)

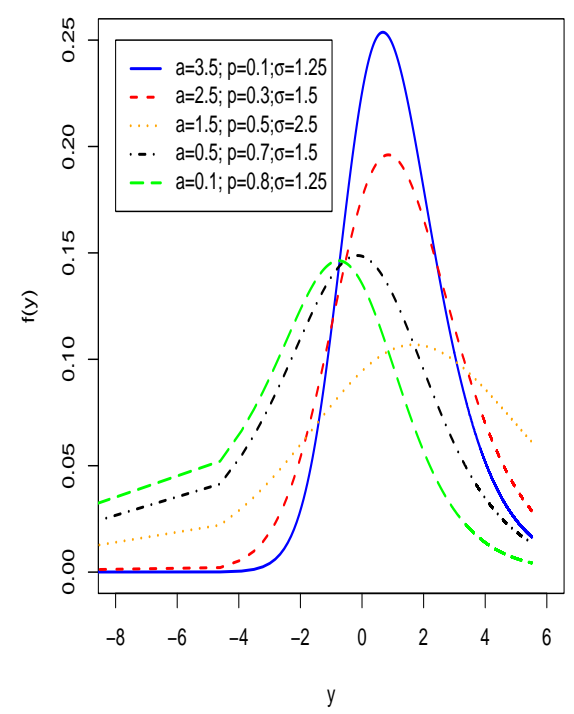

Figura 15 - Gráficos da fdp da distribuição LEGI, para alguns valores dos parâmetros. (a) Para diferentes valores de $a$ e $p$, com $\mu=0$ e $\sigma=1$. (b) Para diferentes valores de $a, p$ e $\mu$, com $\sigma=1$. (c) Para diferentes valores de $a, p, \sigma$ e $\mu=-1$

combinação de distribuições logísticas exponenciadas. Partindo da expressão (56), o termo que está elevado à potência -2 , pode ser escrito como sendo $z=1-G(y)$, em que $G(y)$ é a fda da LE dada pela equação (55) em termos da variável $Y$. Assim, inserindo 
(55) em (21), obtém-se

$$
(1-p z)^{-2}=\sum_{j=0}^{\infty}(j+1) p^{j}\left\{1-\left[1+\exp \left(-\frac{y-\mu}{\sigma}\right)\right]^{-a}\right\}^{j},
$$

utilizando a equação (58) na expressão de (56) da densidade LEGI, obtém-se

$$
\begin{aligned}
f(y)= & (1-p) \frac{a}{\sigma} \exp \left(\frac{y-\mu}{\sigma}\right)\left[1+\exp \left(-\frac{y-\mu}{\sigma}\right)\right]^{-(a-1)}\left[1+\exp \left(\frac{y-\mu}{\sigma}\right)\right]^{-2} \times \\
& \sum_{j=0}^{\infty}(j+1) p^{j}\left\{1-\left[1+\exp \left(-\frac{y-\mu}{\sigma}\right)\right]^{-a}\right\}^{j},
\end{aligned}
$$

levando em consideração a fdp da LE, expressa por

$$
g(y)=\frac{a}{\sigma} \exp \left(\frac{y-\mu}{\sigma}\right)\left[1+\exp \left(-\frac{y-\mu}{\sigma}\right)\right]^{-(a-1)}\left[1+\exp \left(\frac{y-\mu}{\sigma}\right)\right]^{-2}
$$

de (59), obtêm-se a expansão para a fdp da LEGI como uma combinação linear das distribuições logísticas exponenciadas. Assim, a expansão da fdp é dada por

$$
f(y)=(1-p) g(y) \sum_{j=0}^{\infty} w_{j}[1-G(y)]^{j}
$$

em que a expressão $[1-G(y)]^{j}$ pode estar representada em termos dos coeficientes binomiais, como $j$ é um inteiro, então pode-se escrever como $[1-G(y)]^{j}=\sum_{i=0}^{j}(-1)^{i}\left(\begin{array}{l}j \\ i\end{array}\right) G(y)^{i}$, e portanto a expansão da fdp da expressão (60) é expressa por

$$
f(y)=(1-p) g(y) \sum_{j=0}^{\infty} \sum_{i=0}^{j} w_{j}(-1)^{i}\left(\begin{array}{l}
j \\
i
\end{array}\right) G(y)^{i},
$$

considerando $w_{j}=(j+1) p^{j}$ da expressão (61) como os pesos ponderados que multiplicam a família das distribuições logísticas exponenciadas.

\section{- Distribuição LEGII}

Utilizando a expressão (57), o termo que está elevado à potência -2, pode ser escrito como, $z=G(y)$, em que $G(y)$ é a fda da LE dada pela equação (55) em termos da variâvel $Y$, considerando a equação (55) na equação (21), obtém-se

$$
(1-p z)^{-2}=\sum_{j=0}^{\infty}(j+1) p^{j}\left[1+\exp \left(-\frac{y-\mu}{\sigma}\right)\right]^{-a j},
$$

utilizando a equação (62) na expressão (57) da densidade LEGI, obtém-se

$$
\begin{aligned}
f(y)= & (1-p) \frac{a}{\sigma} \exp \left(\frac{y-\mu}{\sigma}\right)\left[1+\exp \left(-\frac{y-\mu}{\sigma}\right)\right]^{-(a-1)}\left[1+\exp \left(\frac{y-\mu}{\sigma}\right)\right]^{-2} \times \\
& \sum_{j=0}^{\infty}(j+1) p^{j}\left[1+\exp \left(-\frac{y-\mu}{\sigma}\right)\right]^{-a j},
\end{aligned}
$$


levando em consideração a função densidade da LE, $g(y)$

$$
g(y)=\frac{a}{\sigma} \exp \left(\frac{y-\mu}{\sigma}\right)\left[1+\exp \left(-\frac{y-\mu}{\sigma}\right)\right]^{-(a-1)}\left[1+\exp \left(\frac{y-\mu}{\sigma}\right)\right]^{-2},
$$

a expansão da fdp da expressão (63) é expressa por

$$
f(y)=(1-p) g(y) \sum_{j=0}^{\infty} w_{j} G(y)^{j}
$$

considerando $w_{j}=(j+1) p^{j}$ da expressão (64) como os pesos ponderados que multiplicam a família das distribuições logísticas exponenciadas.

\subsection{Momentos}

Precisa-se enfatizar a necessidade e a importância dos momentos em qualquer análise estatística. Algumas das características mais importantes de uma distribuição podem ser estudadas pelos momentos. Os momentos ordinários da distribuição logística exponenciada geométrica também podem ser obtidos em termos da expansão da função densidade. Assim, para uma variável $Y$ que segue uma distribuição LEGI ou LEGII, o momento ordinário é definida por $\mu_{r}^{\prime}=E\left(Y^{r}\right)=\int_{-\infty}^{\infty} y^{r} f(y) d y$, em que $f(y)$ está dada por (61) ou (64), respectivamente.

- Distribuição LEGI

$$
\mu_{r}^{\prime}=(1-p) \sum_{j=0}^{\infty} \sum_{i=0}^{j} w_{j}(-1)^{i}\left(\begin{array}{l}
j \\
i
\end{array}\right) \frac{1}{(i+1)} \int_{0}^{1}\left\{\mu-\sigma \log \left[\frac{1-k^{1 / a(i+1)}}{k^{1 / a(i+1)}}\right]\right\}^{r} d k
$$

- Distribuição LEGII

$$
\mu_{r}^{\prime}=(1-p) \sum_{j=0}^{\infty} w_{j} \frac{1}{(j+1)} \int_{0}^{1}\left\{\mu-\sigma \log \left[\frac{1-k^{1 / a(j+1)}}{k^{1 / a(j+1)}}\right]\right\}^{r} d k
$$

A partir dos momentos das expressões (65) ou (66), podem ser obtidas a média, a variância, a assimetria e a curtose destas distribuições.

\subsection{Função Geradora de Momentos}

A função geradora de momentos para a variável $Y$ da distribuição LEGI ou LEGII, definida por $M_{Y}(t)=E[\exp (t Y)]=\int_{-\infty}^{\infty} \exp (t y) f(y) d y$, com fdp (61) ou (64), respectivamente, é dada por

$$
M_{Y}(t)=\sum_{r=0}^{\infty} \frac{t^{r}}{r !} \mu_{r}^{\prime},
$$

em que $\mu_{r}^{\prime}$ é dada por (65) ou (66), são os momentos da distribuição LEGI ou LEGII, respectivamente. 


\subsection{Modelos de Regressão}

Na prática existem situações em que uma ou mais covariáveis estão associadas ao tempo de vida. Por exemplo, na indústria o tempo de vida de um determinado equipamento pode ser influenciado pelo nível de voltagem a que o equipamento é submetido; na área médica, o tempo de vida de um paciente pode estar relacionado com o tipo de tumor, tamanho do mesmo, quantidade de hemoglobina na sangue, raça, idade do paciente, dentre outros fatores.

Considere $X$ uma variável aleatória representando tempo até a falha de um indivíduo e seja $\mathbf{v}=\left(\mathrm{v}_{i 1}, \ldots, \mathrm{v}_{i q}\right)^{T}$ um vetor de $q$ variáveis explanatórias ou covariáveis que podem ser quantitativas ou qualitativas. Uma maneira de determinar a relação entre $X$ e $\mathbf{v}$ é por meio de um modelo de regressão. Existem duas classes importantes de modelos de regressão em análise de sobrevivência: Os modelos de riscos proporcionais para $X$ e o modelo de locação e escala para o logaritmo de $X$. Uma descrição detalhada sobre modelos de riscos proporcionais pode ser obtido em Kalbfleich e Prentice (2002), Lee e Wang (2003), Collet (2003), entre outros. A segunda classe de modelos consiste em utilizar a transformação logarítmica nos tempos de falha de tal forma que para dado um vetor de covariáveis o logaritmo do tempo de falha tem uma distribuição com um parâmetro de locação $\mu$ e um parâmetro de escala $\sigma$.

\subsubsection{Modelo de locação e escala}

Um modelo log-linear ou modelo de locação e escala é escrito da seguinte forma

$$
\boldsymbol{Y}=\mu(\mathbf{v})+\sigma \boldsymbol{Z}
$$

em que $\boldsymbol{Y}$ segue uma distribuição de probabilidade que pertence à família de distribuições que se caracteriza por possuir um parâmetro de locação $\mu(-\infty<\mu<\infty)$, um parâmetro de escala $\sigma>0$ e $\boldsymbol{Z}$ é o erro estocástico. Além disso, a função de sobrevivência de $\boldsymbol{Y}$ dado v é da forma $S\left(\frac{y-\mu(\mathbf{v})}{\sigma}\right)$ em que $S($.) é a função de sobrevivência de $\boldsymbol{Z}$.

Em muitas situações, a maneira geral, de assumir o parâmetro de locação é escrito como $\mu(\mathbf{v})=\mathbf{v}^{T} \boldsymbol{\beta}$, em que $\boldsymbol{\beta}=\left(\beta_{1}, \ldots, \beta_{q}\right)^{T}$ é um vetor de parâmetros desconhecidos. Com esta suposição, obtêm-se

$$
\boldsymbol{Y}=\mathbf{v}^{T} \boldsymbol{\beta}+\sigma \boldsymbol{Z}
$$

tornando-se, o modelo linear para $\boldsymbol{Y}$ com resíduo $\boldsymbol{Z}$. 


\subsubsection{Modelo de regressão logístico exponenciada geométrico}

Como foi visto na seção anterior, em muitas situações práticas, os tempos de vida $x_{i}$ são afetados pelas variáveis explanatórias tais como: nível de colesterol, pressão da sangue e muitos outros fatores. Assim, seja $\mathbf{v}_{i}=\left(\mathrm{v}_{i 1}, \ldots, \mathrm{v}_{i q}\right)^{T}$ o vetor de covariáveis associado à $i$-ésima resposta da variável $y_{i}$ para $i=1, \ldots, n$.

Consideremos uma amostra $\left(y_{1}, \mathbf{v}_{1}\right), \ldots,\left(y_{n}, \mathbf{v}_{n}\right)$ de $n$ observações independentes, em que cada resposta é definida por $y_{i}=\min \left\{\log \left(x_{i}\right), \log \left(c_{i}\right)\right\}$, em que $\log \left(x_{i}\right)$ e $\log \left(c_{i}\right)$, é o logaritmo do tempo de vida e o logaritmo do tempo de censura, respectivamente. Considere também, que a censura é não-informativa e que os tempos de vida observados e de censura são independentes.

Supondo uma relação linear entre $Y$ e v, dada pela expressão (67), pode-se construir um modelo de regressão linear para a variável resposta $y_{i}$, baseado nas distribuições LEGI e LEGII, dada por

$$
y_{i}=\mathbf{v}_{i}^{T} \boldsymbol{\beta}+\sigma z_{i}, \quad i=1, \ldots, n,
$$

em que o erro aleatório $z_{i}$ tem função densidade dada pela expressão (68) ou (69) respectivamente,

- Distribuição LEGI

$$
\begin{aligned}
\pi(z)= & (1-p) a \exp (z)[1+\exp (-z)]^{-(a-1)}[1+\exp (z)]^{-2} \\
& \times\left\{1-p\left\{1-[1+\exp (-z)]^{-a}\right\}\right\}^{-2}, \quad-\infty<z<\infty
\end{aligned}
$$

- Distribuição LEGII

$$
\begin{aligned}
\pi(z)= & (1-p) a \exp (z)[1+\exp (-z)]^{-(a-1)}[1+\exp (z)]^{-2} \\
& \times\left\{1-p[1+\exp (-z)]^{-a}\right\}^{-2}, \quad-\infty<z<\infty
\end{aligned}
$$

em que $Z=(Y-\mu) / \sigma$ é uma variável aleatória padronizada e $\boldsymbol{\theta}=\left(a, p, \sigma, \boldsymbol{\beta}^{T}\right)^{T}$ é o vetor de parâmetros do modelo de regressão.

\subsection{Estimação dos parâmetros}

Após assumir um modelo de regressão que seja adequado para a análise de dados, o próximo passo é encontrar um método para estimar seus parâmetros e realizar o processo de inferência. Em geral, no caso de dados de sobrevivência, essa análise torna-se mais complicada 
quando se faz necessário incorporar observações censuradas, mesmo quando o mecanismo de censura é simples. Nesta seção, será considerado o processo de estimação dos parâmetros pelo método da máxima verossimilhança.

Considere $\left(y_{1}, \mathbf{v}_{i 1}, \delta_{1}\right), \ldots,\left(y_{n}, \mathbf{v}_{i n}, \delta_{n}\right), n$ observações independentes, em que, $y_{i}=\min \left\{\log \left(x_{i}\right), \log \left(c_{i}\right)\right\}$, representa o logaritmo do tempo de falha ou censura, $\mathbf{v}_{i}=$ $\left(\mathbf{v}_{i 1}, \ldots, \mathbf{v}_{i q}\right)^{T}$ é o vetor de covariáveis e $\delta_{i}$ é o indicador de censura, para $i=1, \ldots, n$. Assim, o logaritmo da função de verossimilhança para o vetor de parâmetros $\boldsymbol{\theta}=\left(a, p, \sigma, \boldsymbol{\beta}^{T}\right)^{T}$, é dado por

$$
l(\boldsymbol{\theta})=\sum_{i \in F} \log \left[f\left(y_{i}\right)\right]+\sum_{i \in C} \log \left[S\left(y_{i}\right)\right]
$$

em que $f(y)$ e $S(y)$ são as funções densidade e de sobrevivência da variável aleatória $Y, F$ é o conjunto de observações não censuradas e $C$ denota o conjunto de observações censuradas.

O logaritmo da função de verossimilhança para o vetor de parâmetros $\boldsymbol{\theta}=$ $\left(a, p, \sigma, \boldsymbol{\beta}^{T}\right)^{T}$, dos modelos LEGI e LEGII, são expressos por

- Modelo LEGI

$$
\begin{aligned}
l(\boldsymbol{\theta})= & r \log \left[\frac{a(1-p)}{\sigma}\right]+a \sum_{i \in F}\left(\frac{y_{i}-\mathbf{v}_{i}^{T} \beta}{\sigma}\right)-(a+1) \sum_{i \in F} \log \left[1+\exp \left(\frac{y_{i}-\mathbf{v}_{i}^{T} \beta}{\sigma}\right)\right]- \\
& 2 \sum_{i \in F} \log \left\{1-p\left\{1-\left[1+\exp \left[-\left(\frac{y_{i}-\mathbf{v}_{i}^{T} \beta}{\sigma}\right)\right]\right]^{-a}\right\}\right\}+ \\
& \sum_{i \in C} \log \left\{1-\left[1+\exp \left[-\left(\frac{y_{i}-\mathbf{v}_{i}^{T} \beta}{\sigma}\right)\right]\right]^{-a} \times\right. \\
& {\left.\left[1-p\left\{1-\left[1+\exp \left[-\left(\frac{y_{i}-\mathbf{v}_{i}^{T} \beta}{\sigma}\right)\right]\right]^{-a}\right\}\right]^{-1}\right\} . }
\end{aligned}
$$

- Modelo LEGII

$$
\begin{aligned}
l(\boldsymbol{\theta})= & r \log \left[\frac{a(1-p)}{\sigma}\right]+a \sum_{i \in F}\left(\frac{y_{i}-\mathbf{v}_{i}^{T} \beta}{\sigma}\right)-(a+1) \sum_{i \in F} \log \left[1+\exp \left(\frac{y_{i}-\mathbf{v}_{i}^{T} \beta}{\sigma}\right)\right]- \\
& 2 \sum_{i \in F} \log \left\{1-p\left[1+\exp \left[-\left(\frac{y_{i}-\mathbf{v}_{i}^{T} \beta}{\sigma}\right)\right]\right]^{-a}\right\}+ \\
& \sum_{i \in C} \log \left\{1-(1-p)\left[1+\exp \left[-\left(\frac{y_{i}-\mathbf{v}_{i}^{T} \beta}{\sigma}\right)\right]\right]^{-a} \times\right. \\
& \left.\left\{1-p\left[1+\exp \left[-\left(\frac{y_{i}-\mathbf{v}_{i}^{T} \beta}{\sigma}\right)\right]\right]^{-a}\right\}^{-1}\right\} .
\end{aligned}
$$

em que, $r$ é o número de falhas.

Portanto, os componentes do vetor escore $\boldsymbol{U}=\left(\frac{\partial l(\boldsymbol{\theta})}{\partial a}, \frac{\partial l(\boldsymbol{\theta})}{\partial p}, \frac{\partial l(\boldsymbol{\theta})}{\partial \sigma}, \frac{\partial l(\boldsymbol{\theta})}{\partial \beta_{k}}\right)^{T}$ são mostrados no 
Apêndice B.1. O estimador de máxima verossimilhança (EMV) $\hat{\boldsymbol{\theta}}$ de $\boldsymbol{\theta}$ é obtido numéricamente a partir das equações não lineares

$$
\frac{\partial l(\boldsymbol{\theta})}{\partial a}=\frac{\partial l(\boldsymbol{\theta})}{\partial p}=\frac{\partial l(\boldsymbol{\theta})}{\partial \sigma}=\frac{\partial l(\boldsymbol{\theta})}{\partial \beta_{k}}=0, \quad \text { para } k=1, \ldots, q .
$$

Porém, para obter o estimador de MV para os parâmetros $a, p, \sigma$ e $\beta_{k}$ é necessário a utilização de métodos iterativos numéricos, por exemplo, Newton-Raphson ou o algoritmo de Quase-Newton. Os métodos iterativos para iniciar o algoritmo especificam uma estimativa inicial $\boldsymbol{\theta}^{(0)}$, iterá-la sucessivamente até obter a convergência. A função de sobrevivência estimada para $y_{i}$ é dada por

- Modelo LEGI

$$
\begin{aligned}
S\left(y_{i} ; \hat{\sigma}, \hat{\beta}, \hat{a}, \hat{p}\right)= & 1-\left[1+\exp \left[-\left(\frac{y_{i}-\mathbf{v}_{i}^{T} \hat{\beta}}{\hat{\sigma}}\right)\right]\right]^{-\hat{a}} \times \\
& \left\{1-\hat{p}\left[1-\left[1+\exp \left[-\left(\frac{y_{i}-\mathbf{v}_{i}^{T} \hat{\beta}}{\hat{\sigma}}\right)\right]\right]^{-\hat{a}}\right]\right\}^{-1} .
\end{aligned}
$$

- Modelo LEGII

$$
\begin{aligned}
S\left(y_{i} ; \hat{\sigma}, \hat{\beta}, \hat{a}, \hat{p}\right)= & 1-(1-\hat{p})\left[1+\exp \left[-\left(\frac{y_{i}-\mathbf{v}_{i}^{T} \hat{\beta}}{\hat{\sigma}}\right)\right]\right]^{-\hat{a}} \times \\
& \left\{1-\hat{p}\left[1+\exp \left[-\left(\frac{y_{i}-\mathbf{v}_{i}^{T} \hat{\beta}}{\hat{\sigma}}\right)\right]\right]^{-\hat{a}}\right\}^{-1} .
\end{aligned}
$$

No caso em que o tamanho da amostra é grande e sob certas condições de regularidade para a função de verossimilhança, intervalos de confiança e testes de hipóteses podem ser obtidos utilizando o fato de que a distribuição assintótica dos estimadores de máxima verossimilhança é a distribuição normal com média $\boldsymbol{\theta}$ e matriz de covariância dada pela inversa da matriz de informação esperada ou de Fisher. Portanto, a matriz de covariância assintótica é dada por $I(\boldsymbol{\theta})^{-1}$ em que $\boldsymbol{I}(\boldsymbol{\theta})=E[\boldsymbol{J}(\boldsymbol{\theta})]$ e $\boldsymbol{J}(\boldsymbol{\theta})=-\left\{\frac{\partial^{2} l(\boldsymbol{\theta})}{\partial \boldsymbol{\theta} \partial \boldsymbol{\theta}^{T}}\right\}$.

Como não é possível determinar a matriz de informação de Fisher $I(\boldsymbol{\theta})$ devido a presença de observações censuradas, uma forma alternativa é utilizar a matriz de informação observada $J(\boldsymbol{\theta})$, avaliada no estimador de máxima verossimilhança $\boldsymbol{\theta}=(\hat{\boldsymbol{\theta}})$, esta aproximação normal assintótica de $(\hat{\boldsymbol{\theta}})$ é dada por

$$
\sqrt{n}(\hat{\boldsymbol{\theta}}-\boldsymbol{\theta}) \sim \mathrm{N}_{q+2}\left(0, \boldsymbol{J}(\boldsymbol{\theta})^{-1}\right),
$$


em que $J(\boldsymbol{\theta})$ é a matriz de informação observada $(q+2)(q+2)$ dada por

$$
\boldsymbol{J}(\boldsymbol{\theta})=-\left(\begin{array}{cccc}
J_{a a} & J_{a p} & J_{a \sigma} & J_{a \beta_{k}} \\
J_{p a} & J_{p p} & J_{p \sigma} & J_{p \beta_{k}} \\
J_{\sigma a} & J_{\sigma p} & J_{\sigma \sigma} & J_{\sigma \beta_{k}} \\
J_{\beta_{k} a} & J_{\beta_{k} p} & J_{\beta_{k} \sigma} & J_{\beta_{k} \beta_{l}}
\end{array}\right) \text {, para } k, l=1, \ldots, q
$$

A matriz de informação observada $J(\boldsymbol{\theta})$, é mostrado no apêndice B.2. Assim, o intervalo de confiança assintótico, com coeficiente de confiança $\gamma$ para cada parâmetro $\theta_{r}$ é obtido por meio da expressão

$$
A C I\left(\theta_{r}, 100(1-\gamma) \%\right)=\left(\hat{\theta}_{r}-z_{\gamma / 2} \sqrt{\hat{J}^{\theta_{r}, \theta_{r}}} \quad, \hat{\theta}_{r}+z_{\gamma / 2} \sqrt{\hat{J}^{\theta_{r}, \theta_{r}}}\right)
$$

em que, $\hat{J}^{\theta_{r}, \theta_{r}}$ é o $r$-ésimo elemento diagonal estimado a partir de $\boldsymbol{J}(\hat{\boldsymbol{\theta}})^{-1}$ e $z_{\frac{\gamma}{2}}$ é o quantil $1-\frac{\gamma}{2}$ da distribuição normal padrão. 


\section{APLICAÇÕES}

As aplicações descritas a seguir têm como por objetivo mostrar a utilidade dos modelos LLEGI, LLEGII, LEGI e LEGII, no qual são apresentadas duas aplicações baseadas em dados reais.

O primeiro conjunto de dados analisados na Seção 5.1, refere-se a dados de contaminação ambiental da cidade de Santiago do Chile, em que é medida a quantidade de concentração de dióxido de enxofre $\left(\mathrm{SO}_{2}\right)$ no ar, em uma amostra de 744 observações, informação previamente estudada por Balakrishnam et al. (2009). O modelo ajustado a esse banco de dados foi a distribuição LLEGII.

O segundo conjunto de dados analisados na Seção 5.2, refere-se ao tempo de falha de amostras de isolante elétrico da resina epóxi submetidas a três níveis de voltagem, descrito em Lawless (2003). Foram analisados $n=60$ isolantes divididos em três grupos de voltagem de $52.5,55.0$ e 57.5 kilovolts $(\mathrm{kV})$.

Os softwares utilizados para o cálculo das estimativas dos parâmetros das distribuições, foram o R Development Core Team (2009) e o SAS- (Statistical Analysis SystemInstitute,2004).

\subsection{Aplicação 1: Dados de contaminação do ambiente}

Nesta seção utilizou-se um conjunto de dados reais ( $n=744$ observações) relacionada à contaminação ambiental, previamente estudado por Balakrishnnam et al. (2009).

A cidade de Santiago do Chile é reconhecida como uma das cidades mais contaminadas do ambiente no mundo. Nesse contexto, a Comissão Nacional de Meio Ambiente do Governo do Chile, com o objetivo de medir o nível de poluição atmosférica e seus efeitos adversos associados à saúde sobre os seres humanos em Santiago, recolheu dados sobre as concentrações de dióxido de enxofre $\left(\mathrm{SO}_{2}\right)$ no ar. Esses dados foram utilizados para ajustar as duas distribuições LLEGI e LLEGII, como também seus sub-modelos: as distribuições LLE $(p=0)$ e $\operatorname{LL}(p=0, a=1)$.

Em primeiro lugar, descreveu-se o conjunto de dados; em seguida, encontrou-se os EMV's dos parâmetros com seu correspondente erro padrão entre parênteses e os valores das estatísticas do AIC (Critério de Informação de Akaike), CAIC (Critério de Informação de Akaike Corrigido) e BIC (Critério de Informação Bayesiano), em que o menor valor deste critério, determina o melhor ajuste. Observou-se que a reparametrização é penalizado usando 
esses critérios de modo que os parâmetros adicionais nas distribuições LLEGI e LLEGII não conduzem necessariamente a um menor valor das estatísticas AIC, CAIC ou BIC. Em cada caso, os parâmetros são estimados por meio do método de máxima verossimilhança descritos na Seção 3.5, utilizando o procedimento NLMixed do SAS. Em seguida, realizou-se os testes de razão da verossimilhança apresentados na Seção 3.5.1. Finalmente, construi-se o histograma desses dados para fornecer uma comparação visual das funções de densidade ajustadas.

Tabela 1 - Estatísticas descritivas

\begin{tabular}{ccccccccc}
\hline Media & Mediana & Moda & SD & Variáncia & Assimetria & Curtose & Min. & Max. \\
\hline 2,926 & 2,00 & 2,00 & 2,015 & 4,060 & 4,339 & 34,914 & 1,00 & 25,00 \\
\hline
\end{tabular}

A Tabela 1, mostra o resumo descritivo do conjunto total de dados os quais os números indicam que têm assimetria positiva e curtose. A maximização iterativa do logaritmo da função de verossimilhança, começa com valores iniciais para $\alpha$ e $\beta$ que são tomados do ajuste da distribuição LL com $a=1$ e $p=0$. Essas estimativas dos parâmetros com os correspondentes erros padrões em parênteses, são dadas na Tabela 2, para os diversos modelos ajustados, além de fornecer os valores das medidas AIC, BIC e CAIC. Esses resultados indicam que o modelo LLEGII com seus três parâmetros de forma $(a, p, \beta)$ e um parâmetro de escala $(\alpha)$, apresenta o menor valor para AIC, BIC e CAIC entre todos os modelos ajustados e portanto, pode ser escolhido como o modelo mais adequado.

Para obter uma breve avaliação da qualidade de ajuste dos dados, foi realizado o teste da razão de verossimilhança descritos na Seção 3.5.1 para testar alguns sub-modelos das distribuições LLEGI e LLEGII. A Tabela 3 mostra os resultados dos testes da razão de verossimilhança, fornecendo indicações favoráveis ao modelo LLEGII.

Portanto, com a finalidade de avaliar se o modelo LLEGII é apropriado, é fornecido por meio da comparação visual do histograma dos dados de contaminação $X$, conjuntamente com sua fdp $f(x)$ ajustada da distribuição LLEGII que é mostrado na Figura 16a, mostra-se também o gráfico da função de distribuição acumulada $F(x)$ da distribuição LLEGII e a fda empírica dos dados mostrados na Figura 16b. Assim, concluimos que a distribuição LLEGII fornece um bom ajuste para estes dados em base nos resultados obtidos anteriormente. 
Tabela 2 - Estimativas de máxima verossimilhança dos parâmetros do modelo para dados de contaminação e o critério de informação

\begin{tabular}{c|cccc|ccc}
\hline Modelo & $a$ & $p$ & $\alpha$ & $\beta$ & AIC & CAIC & BIC \\
\hline LLEGI & 1,5314 & 0,6279 & 2,7022 & 2,8671 & 2512,1 & 2512,2 & 2530,5 \\
& $(0,2191)$ & $(0,3455)$ & $(0,7396)$ & $(0,2582)$ & & & \\
LLEGII & 19,8288 & 0,9190 & 0,4339 & 3,1975 & 2501,8 & 2501,9 & 2520,3 \\
& $(18,99)$ & $(0,0268)$ & $(0,1383)$ & $(0,1256)$ & & & \\
LLE & 1,5360 & 0 & 2,0317 & 3,0330 & 2511,0 & 2511,1 & 2524,9 \\
& $(0,2384)$ & - & $(0,1568)$ & $(0,1562)$ & & & \\
LL & 1 & 0 & 2,4710 & 3,4285 & 2518,3 & 2518,4 & 2527,5 \\
& - & - & $(0,0458)$ & $(0,1053)$ & & & \\
\hline
\end{tabular}

Tabela 3 - Teste da razão de verossimilhança

\begin{tabular}{c|c|c|c}
\hline Contaminação & Hipótese & Est. $w$ & $p$-valor \\
\hline LLEGI vs LLE & $H_{0}: p=0$ vs $H_{1}: p \neq 0$ & 0,90 & 0,3428 \\
LLEGI vs LL & $H_{0}: p=0$ e $a=1$ vs $H_{1}: p \neq 0$ e $a \neq 1$ & 11,20 & 0,0008 \\
& $H_{0}: p=0$ vs $H_{1}: p \neq 0$ & 10,20 & 0,0061 \\
LLEGII vs LLE & $H_{0}: p=0$ e $a=1$ vs $H_{1}: p \neq 0$ e $a \neq 1$ & 20,5 & $<0,00001$ \\
LLEGII vs LL
\end{tabular}

\subsection{Aplicação 2: Dados de nível de voltagem}

Nesta seção ilustrou-se os modelos de regressão LEGI e LEGII com uma aplicação referentes aos níveis de voltagem do isolante elétrico da resina epóxi. A resina epóxi é um polímero termoendurecível, que endurece-se quando é misturado com um catalisador e são usadas para várias aplicações: revestimento de proteção, revestimento para ambientes marinhos, de pisos, compostos de moldagem, como materiais isolantes, plásticos reforçados e têxteis.

Lawless (2003) mostra um experimento em que os dados analisados são referentes ao tempo de falha (minutos) de amostras de um isolante elétrico da resina epóxi, os quais foram estudadas em um teste de tensão de vida acelerado. Foram analisados $n=60$ isolantes, divididos em três grupos de nível de voltagem de 52,5; 55,0 e 57,5 kilovolts (kV), a porcentagem de observações censuradas foi de $10 \%$. Os dados foram analisados usando os modelos de 
(a)

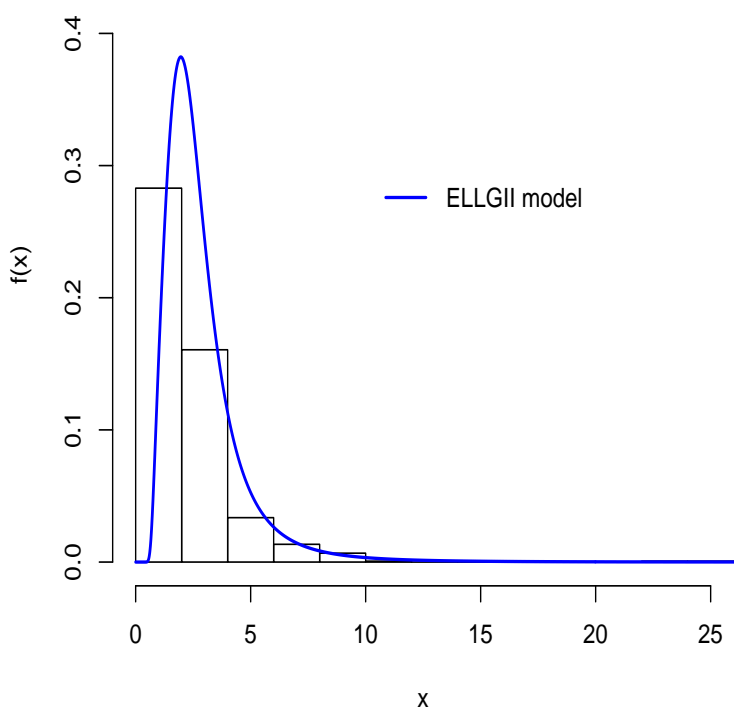

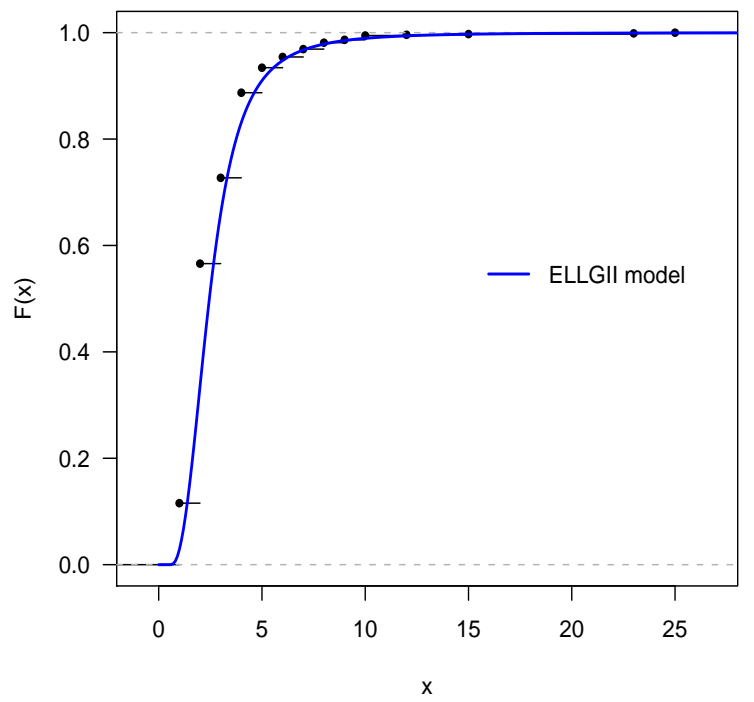

Figura 16 - (a) Densidade LLEGII ajustada para os dados de Contaminação. (b) Função de distribuição acumulada estimado da distribuição LLEGII para os dados de contaminação

regressão LEGI e LEGII, considerando:

$x_{i}$ : tempo em minutos, até a falha do $i$-ésimo isolante elétrico;

$\delta_{i}$ : indicador de censura da $i$-ésima observação;

$\mathbf{v}_{i 1}$ : nível de voltagem em kilovolts $(\mathrm{kV})$.

e a equação

$$
y_{i}=\boldsymbol{\beta}_{0}+\boldsymbol{\beta}_{1} \mathbf{v}_{i 1}+\sigma \boldsymbol{z}_{i},
$$

em que a variável aleatória $Y_{i}=\log \left(X_{i}\right)$ segue a distribuição LEGI ou LEGII, dadas pelas expressões (56) ou (57) respectivamente, $\boldsymbol{z}_{i}$ são os erros aleatórios com fdp expressa por (68) ou (69) segundo seja o caso, para $i=1, \ldots, 60$.

Na Tabela 4, são apresentadas as EMVs dos parâmetros referentes aos modelos de regressão LEGI e LEGII, com seus correspondentes erros padrões que estão entre parênteses e o $p$-valor entre colchetes de cada modelo, os quais foram ajustados aos dados de níveis de voltagem (usando o procedimento NLMixed do SAS) e os correspondentes valores das estatísticas AIC, CAIC e BIC para comparar os modelos de regressão. A maximização iterativa 
do logaritmo da função de verossimilhança, começa com valores iniciais para $\beta$ e $\sigma$ que são tomados do ajuste dos modelos de regressão LEGI e LEGII com $a=1$ e $p=0$.

Tabela 4 - Estimativas de máxima verossimilhança dos parâmetros dos modelos de regressão ajustados aos dados de níveis de voltagem, seus correspondentes EPs (em parênteses), p-valor em [.] e as estatísticas $\mathrm{AIC}, \mathrm{CAIC}$ e BIC

\begin{tabular}{|c|c|c|c|c|c|c|c|c|}
\hline Modelo & $a$ & $p$ & $\sigma$ & $\beta_{0}$ & $\beta_{1}$ & $\mathrm{AIC}$ & CAIC & $\mathrm{BIC}$ \\
\hline \multirow[t]{3}{*}{ LEGI } & 3,0931 & $1 \mathrm{E}-8$ & 0,7057 & 15,0204 & $-0,1739$ & 166,7 & 167,9 & 177,2 \\
\hline & $(4,3257)$ & $(1 \mathrm{E}-9)$ & $(0,1824)$ & $(4,4597)$ & $(0,0663)$ & & & \\
\hline & - & - & - & {$[0,0013]$} & {$[0,0110]$} & & & \\
\hline \multirow[t]{3}{*}{ LEGII } & 3,9296 & 0,9999 & 0,4396 & 13,0640 & $-0,2118$ & 135,8 & 136,9 & 146,3 \\
\hline & $(0,3181)$ & $(0,000092)$ & $(0,0496)$ & $(2,8459)$ & $(0,0493)$ & & & \\
\hline & - & - & - & {$[<0,001]$} & {$[<0,001]$} & & & \\
\hline \multirow[t]{3}{*}{$\mathrm{LE}$} & 3,0931 & 0 & 0,7057 & 15,0204 & $-0,1739$ & 164,7 & 165,5 & 173,1 \\
\hline & $(4,3276)$ & - & $(0,1824)$ & $(4,4598)$ & $(0,0663)$ & & & \\
\hline & - & - & - & {$[0,0013]$} & {$[0,0110]$} & & & \\
\hline \multirow[t]{3}{*}{ Logistico } & 1 & 0 & 0,5296 & 17,8799 & $-0,2072$ & 164,3 & 164,7 & 170,5 \\
\hline & - & - & $(0,0599)$ & $(3,0932)$ & $(0,0560)$ & & & \\
\hline & - & - & - & {$[<0,0001]$} & {$[0,0005]$} & & & \\
\hline
\end{tabular}

Os resultados obtidos dos modelos ajustados são mostrados na Tabela 4, esses resultados indicam que o modelo de regressão LEGII tem os menores valores das estatísticas AIC, CAIC e BIC dentre os modelos ajustados e portanto, pode ser escolhido como o melhor modelo, o qual indica que ajusta-se com maior flexibilidade aos dados. Observa-se também, que a estimativa do parâmetro $p$ para o modelo LEGI é quase zero $(\hat{p}=0)$, indicando assim que os modelos LEGI e LE são quase equivalentes.

Segundo esses resultados da Tabela 4, pode-se obter o modelo de regressão final que é dado por

$$
y_{i}=13,0640-0,2118 \mathbf{v}_{i 1}+0,4396 \boldsymbol{z}_{i}
$$

Portanto, a partir do modelo de regressão ajustada LEGII com $\mathbf{v}_{1}$ é significativo a 1\%, mas existe uma diferença significativa entre os níveis de voltagem de 52,5; 55,0 e 57,5 para os dados de tempo de falha de isolantes elétricos.

Na Figura 17, apresenta-se o gráfico das curvas da função de sobrevivência esti- 


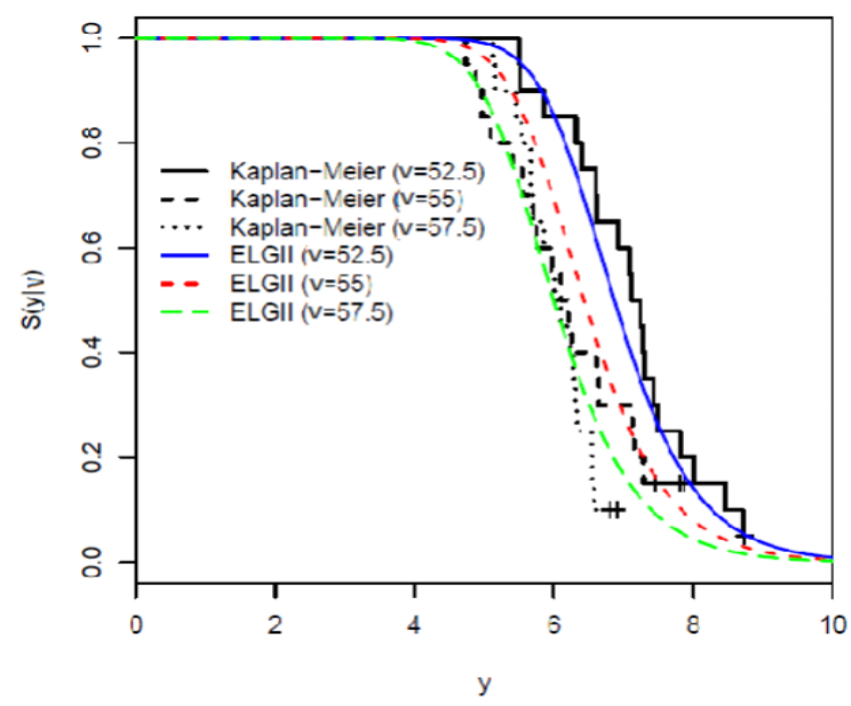

Figura 17 - Curvas da função de sobrevivência estimada para o modelo LEGII para os dados de níveis de voltagem

mada para os diferentes níveis de voltagem, um com $\mathbf{v}_{1}=52,50$ outro com $\mathbf{v}_{1}=55,0$ e outro com $\mathbf{v}_{1}=57,50$. De modo geral, pode-se concluir, que o tempo de falha estimado dos isolantes elétricos diminui à medida que são submetidos a um nível de voltagem maior.

A fim de fazer uma breve avaliação da qualidade de ajuste dos dados são realizados testes da razão de verossimilhança para testar os sub-modelos LE e logístico em relação ao modelo LEGII. A Tabela 5 mostra os resultados dos testes da razão de verossimilhança, fornecendo indicações favoráveis ao modelo LEGII.

Tabela 5 - Teste da razão de verossimilhança

\begin{tabular}{c|c|c|c}
\hline Voltagem & Hipótese & Est. $w$ & $p$-valor \\
\hline LEGII vs LE & $H_{0}: p=0$ vs $H_{1}: p \neq 0$ & 30,90 & $<0,00001$ \\
LEGII vs Logistico & $H_{0}: p=0$ and $a=1$ vs $H_{1}: p \neq 0$ e $a \neq 1$ & 32,50 & $<0,00001$ \\
\hline
\end{tabular}




\section{CONSIDERAÇÕES FINAIS}

\subsection{Conclusões}

Neste trabalho foram propostas duas distribuições novas, baseadas na distribuição log-logística exponenciada proposta por Rosaiah et al. (2006) e seguindo a idéia de Adamidis e Loukas (1998) como um processo de mistura de distribuições, obtiveram-se as distribuições log-logística exponenciada geométrica tipo I e log-logística exponenciada geométrica tipo II. Distribuições importantes para a análise de tempos de vida, pois a principal vantagem dos novos modelos é a flexibilidade em acomodar diferentes formas de função de taxa de falha: crescente, decrescente, unimodal, bimodal e forma de banheira e também acomoda curvas de formas de situações incertas.

Forneceu-se uma descrição detalhada de algumas propriedades matemáticas das distribuições novas, baseadas em expansões da função de densidade. Estes resultados permitem obter expressões para os momentos e para a função geradora de momentos. Calculou-se os desvios médios, as curvas de Bonferroni e Lorenz.

Também foram propostos dois modelos de regressão baseados nas novas distribuições para dados com censura. O método da máxima verossimilhança foi usado para estimar os parâmetros dos modelos, foi obtido a matriz de informação observada e foi realizado os testes de hipóteses para os parâmetros baseados na distribuição assintótica destas estimativas.

Finalmente, foram demostradas em duas aplicações que os modelos propostos podem produzir melhores ajustes que seus sub-modelos.

\subsection{Trabalhos futuros}

Como possíveis trabalhos futuros podem-se considerar os seguintes temas:

1. Realizar um estudo computacional comparando a eficiência de diferentes métodos de estimação como por exemplo bootstrap, jackknife e Bayesiano para os modelos de regressão propostos.

2. Desenvolver medidas de diagnóstico baseado na teoria de influência local e global para modelos de regressão.

3. Propor uma análise de resíduos para os modelos de regressão e mediante simulações estudar a distribuição empírica desses resíduos. 
4. Utilizar os modelos propostos para modelar indivíduos de longa duração. 


\section{REFERÊNCIAS}

ADAMIDIS, K.; LOUKAS, S. A lifetime distribution with decreasing failure rate. Statistics and Probability Letters, Amsterdam, v.39, p.35-42, 1998.

ADAMIDIS, K.; DIMITRAKOPOULOU, T; LOUKAS, S. On an extension of the exponential-geometric distribution. Statistics and Probability Letters, Amsterdam, v.73, n.3, p.259-269, July 2005.

AKAIKE, H. A new look at the statistical model identification. IEEE Transactions on Automatic Control, Boston, v.19, n.6, p.716-723, Dec. 1974.

AHMAD, M.I.; SINCLAIR, C.D.; WERRITTY, A. Log-logistic flood frequency analysis. Journal of Hidrology, Berlin, v.98, p.205-224, 1988.

ALKASASBEH, M.R.; RAQAB, M.Z. Estimation of the generalized logistic distribution parameters: Comparative study. Statistical Methodology, Amsterdam, v.6, n.3, p.262-279, May 2009.

ASHKAR, F.; MAHDI, S. Fitting the log-logistic distribution by generalized moments. Journal of Hydrology, Germany, v.328, n.3-4, p.694-703, Sept. 2006.

BALAKRISHNAN, N.; LEUNG, M.Y. Order statistic from the type I generalized logistic distribution Communications in Statistic-Simulation and Computation, London, v.17, n.1, p.25-50, 1988.

BALAKRISHNAN, N.; LEIVA, V.; SANHUEZA, A.; CABRERA, E. Mixture inverse Gaussian distributions and its transformations, moments and applications. Statistic, Great Britain, v.43, n.1, p.91-104, Feb. 2009.

BARRETO-SOUZA, W.; CRIBARI-NETO, F. A generalization of the exponential-Poisson distribution. Statistics and Probability Letters, Amsterdam, v.79, n.24, p.2493-2500, Dec. 2009.

BARRETO-SOUZA, W.; SANTOS, A.; CORDEIRO, G.M. The beta generalized exponential distribution. Journal of Statistical Computation and Simulation, London, v.80, n.2, p.159-172, Feb. 2010.

BARRETO-SOUZA, W.; LEMOS DE MORAIS, A.; CORDEIRO, G.M. The Weibull-geometric distribution. Journal of Statistical Computation and Simulation, London, v.81, n.5, p.645-657, May 2011.

BOZDOGAN, H. Model selection and Akaikes information criterion (AIC): The general theory and its analytical extensions. Psychometrica, Berlin, n.52, p.345-370, 1987.

CARRASCO, J.M.F.; ORTEGA, E.M.M.; CORDEIRO, G.M. A generalized modified Weibull distribution for lifetime modeling. Computational Statistics and Data Analysis, v.53, p.450-462, 2008a.

CARRASCO, J.M.F.; ORTEGA, E.M.M.; PAULA, G.A. Log-Modified Weibull Regression Models with Censored Data: Sensitivity and Residual Analysis. Computational Statistics and Data Analysis, Amsterdam, v.52, n.8, p.4021-4039, Apr. 2008b.

COlOSIMO, E.A.; GIOlO, S.R. Análise de Sobrevivência Aplicada. São Paulo: Edgar Blucher, 2006. 392p.

COLLETT, D. Modelling survival data in medical research. London: Chapman and Hall, 2003. 391p. 
CORDEIRO, M.G.; ORTEGA, E.M.M.; SILVA, O.G. The Exponentiated Generalized Gamma Distribution with Application to Lifetime Data. Journal of Statistical Computation and Simulation, London, v.81, n.7, p.827-842, July 2011.

CORDEIRO, M.G.; CASTRO, M. A new family of generalized distributions. Journal of Statistical Computation and Simulation, London, v.81, n.7, p.883-898, July 2011.

GUPTA, R.D.; KUNDU, D. Generalized exponential distributions Australian and new Zealand Journal of Statistic, Austrália, v.41, n.2, p.173-188, 1999.

GUPTA, R.D.; KUNDU, D. Exponentiated Exponential Family:An Alternative to Gamma and Weibull Distributions. Biometrical Journal, Berlin, v.43, n.1, p.117-130, 2001.

GUPTA, R.D.; KUNDU, D. Generalized exponential distribution: Existing results and some recent developments. Journal of Statistical Planning and Inference, Amsterdam, v.137, n.11, p.3537-3547, Nov. 2007.

HASHIMOTO, E.M.; ORTEGA, E.M.M.; CANCHO, V.G.; CORDEIRO, G.M. The log-exponentiated Weibull regression model for interval-censored data. Computational Statistics and Data Analysis, Amsterdam, v.54, n.4, p.1017-1035, Apr. 2010.

HOSKING, J.R.M.; WALLIS, J.R.; WOOD, E.F. Estimation of the generalized extreme value distribution by the method of Probability Weighted Moments. Technometrics, New York, v.27, n.3, Aug. 1985.

KALBFLEISH, J.D.; PRENTICE, R.L. The Statistical analysis of Failure Time Data. 2nd ed. New York: John Wiley, 2002. 439p.

KLEIBER, C.; KOTZ, S. Statistical Size Distributions in Economics and Actuarial Sciencies. Oklahoma, John Wiley Professio, 2003. 332p.

KIRSHENBAUM, A.P.; OLSEN, D.M.; BICKEL, W.K. A quantitative review of the ubiquitous relapse curve. Journal of Substance Abuse Treatment, New York, v.36, p.8-17, 2009.

KUNDU, D.; RAQAB, M. Generalized Rayleigh distribution: different methods of estimations. Computational Statistics and Data Analysis, Amsterdam, v.49, n.1, p.187-200, Apr. 2005.

KUS, C. A new lifetime distribution.Computational Statistics and Data Analysis, Amsterdam, v.51, n.9, p.4497-4509, 2007.

LAWLESS, J.F. Statistical Models and Methods for lifetime data. New York:John Wiley, 2003. 630p.

LEE, E.T.; WANG, J.W. Statistical methods for survival data analysis. 3rd ed. Oklahoma: Wiley - Interscience, 2003. 527p.

LEROY, R.; BOGAERTS, K.; LESAFRE, E.; DECLERCK, D. The emergence of permanent teeth in Flemsih children. Community Dentistry and Oral Epidemiology, New York, v.31, n.1, p.30-39, Feb. 2003.

MARSHALL, A.W.; OLKIN, I. A new method for adding a parameter to a family of distribution to the exponential and Weibull families. Biometrika, Great Britain, v.84, n.3, p.641-652, 1997.

MUDHOLKAR, G.S.; SRIVASTAVA, D.K.; FREIMER, M. The Exponentiated Weibull Family: A Reanalysis of the Bus-Motor-Failure Data. Technometrics, Alexandria, v.37, n.4, p.436-445, Nov. 1995. 
MUDHOLKAR, G.S.; SRIVASTAVA, D.K.; KOLLIA, G.D. A Generalization of the Weibull Distribution with Application to the Analysis of Survival Data. Journal of the American Statistical Association, New York, v.91, n.436, p.1575-1583, Dec. 1996.

NADARAJAH, S.; KOTZ, S. The Beta Gumbel distribution.Mathematical Problems in Engineering, New York, v.4, p.323-332, 2004.

NADARAJAH, S. The exponentiated Gumbel distribution with climate application.

Environmetrics, Great Britain, v.17, n.1, p.13-23, Feb. 2006.

NADARAJAH, S.; KOTZ, S. The beta exponential distribution. Reliability Engineering and System Safety, Great Britain, v.91, n.6, p.689-697, 2006a.

NADARAJAH, S.; KOTZ, S. The exponentiated type distributions. Acta Applicandae Mathematicae, Amsterdam, v.92, n.2, p.97-111, 2006b.

OJO, M.O.; OLAPADE, A.K. On the generalized logistic and Log-logistic distributions. Kragujevac Journal Mathematics, Belgrado, v.25, p.65-73, 2003.

PROSCHAN, F. Theoretical Explanation of Observed Decreasing Failure Rate.

Technometrics, New York, v.5, n.3, p.375-383, Aug. 1963.

RODRIGUES, C. Distribuições em série de potências modificadas inflacionadas e distribuição Weibull binomial negativa. 2011. 111p. Dissertação (Mestrado em Estatística e Experimentação Agronômica) - Escola Superior de Agricultura "Luiz de Queiroz", Universidade de São Paulo, Piracicaba, 2011.

ROSAIAH, K.; KANTAM R.R.L.; KUMAR, S. Reliability test plans for exponentiated log-logistic distribution. Economic Quality Control, Berlin, v.21, n.2, p.279-289, 2006.

SANTANA, T.V.F. de. As distribuições Kumaraswamy-log-logística e

Kumaraswamy-logística. 2010. 104p. Dissertação (Mestrado em Estatística e Experimentação Agronômica) - Escola Superior de Agricultura "Luiz de Queiroz", Universidade de São Paulo, Piracicaba, 2010.

SCHWARZ, G. Estimating the dimension of a model. The Annals of Statistics, New York, v.6, n.2, p.461-464, 1978.

SILVA, G.O. Modelos de regressão quando a função de taxa de falha não-monótona e o modelo probabilístico beta Weibull modificada. 2008. 207p. Tese (Doutorado em Agronomia) - Escola Superior de Agricultura "Luiz de Queiroz",

Universidade de São Paulo, Piracicaba, 2008.

SILVA, R.B.; BARRETO-SOUZA, W.; CORDEIRO, M.C. A new distribution with decreasing, increasing and upside-down bathtub failure rate. Computationals Statistics and Data Analysis, Amsterdam, v.54, n.4, p.935-944, Apr. 2010.

TADIKAMALLA, P.R.; JOHNSON, N.L. Systems of frequency curves generated by transformations of logistic variables. Biometrika, London, v.69, n.2, p.461-465, 1982.

TADIKAMALLA, P.R. A look at the Burr and related distributions. International Statistical Review, v.48, p.337-344, Great Britain, 1980.

WEIBULL, W. A statistical distribution of wide applicability. Journal of Applied Mechanics, New York, v.18, p.293-297, 1951. 
APÊNDICES 


\section{APÊNDICE A - Matriz de informação observada $\boldsymbol{J}(\boldsymbol{\theta})$}

Derivadas parciais de segunda ordem em relação aos parâmetros das distribuições LLEGI e LLEGII, considerando $q_{i}=\left[1+\left(\frac{x_{i}}{\alpha}\right)^{-\beta}\right]$

\section{Distribuição LLEGI}

$$
\begin{aligned}
& \boldsymbol{J}_{\alpha \alpha}=-\frac{n \beta}{\alpha^{2}}-\frac{(a+1) \beta}{\alpha^{2}} \sum_{i=1}^{n}\left[\frac{\beta\left(\frac{x_{i}}{\alpha}\right)^{-\beta}}{q_{i}}-\frac{\left(\frac{x_{i}}{\alpha}\right)^{-\beta}}{q_{i}}-\frac{\beta\left(\frac{x_{i}}{\alpha}\right)^{-2 \beta}}{q_{i}^{2}}\right] \\
& -\frac{2 p a \beta}{\alpha^{2}} \sum_{i=1}^{n}\left[\frac{a \beta\left(\frac{x_{i}}{\alpha}\right)^{-2 \beta} q_{i}^{-(a+2)}}{1-p\left(1-q_{i}^{-a}\right)}-\frac{\beta\left(\frac{x_{i}}{\alpha}\right)^{-\beta} q_{i}^{-(a+1)}}{1-p\left(1-q_{i}^{-a}\right)}+\frac{\left(\frac{x_{i}}{\alpha}\right)^{-\beta} q_{i}^{-(a+1)}}{1-p\left(1-q_{i}^{-a}\right)}\right. \\
& \left.+\frac{\beta\left(\frac{x_{i}}{\alpha}\right)^{-2 \beta} q_{i}^{-(a+2)}}{1-p\left(1-q_{i}^{-a}\right)}-\frac{p a \beta\left(\frac{x_{i}}{\alpha}\right)^{-2 \beta} q_{i}^{-2(a+1)}}{1-p\left(1-q_{i}^{-a}\right)}\right] \\
& \boldsymbol{J}_{\alpha a}=-\frac{\beta}{\alpha} \sum_{i=1}^{n} \frac{\left(\frac{x_{i}}{\alpha}\right)^{-\beta}}{q_{i}}-\frac{2 p \beta}{\alpha} \sum_{i=1}^{n}\left[\frac{\left(\frac{x_{i}}{\alpha}\right)^{-\beta} \log \left(q_{i}\right) q_{i}^{-(a+1)}}{1-p\left(1-q_{i}^{-a}\right)}-\frac{a\left(\frac{x_{i}}{\alpha}\right)^{-\beta} q_{i}^{-(a+1)}}{1-p\left(1-q_{i}^{-a}\right)}\right. \\
& \left.-\frac{p\left(\frac{x_{i}}{\alpha}\right)^{-\beta} \log \left(q_{i}\right) q_{i}^{-(2 a+1)}}{\left[1-p\left(1-q_{i}^{-a}\right)\right]^{2}}\right] \\
& \boldsymbol{J}_{\alpha \beta}=\frac{n}{\alpha}-\frac{(a+1)}{\alpha} \sum_{i=1}^{n}\left[\frac{\beta\left(\frac{x_{i}}{\alpha}\right)^{-\beta} \log \left(\frac{x_{i}}{\alpha}\right)}{q_{i}}+\frac{\left(\frac{x_{i}}{\alpha}\right)^{-\beta}}{q_{i}}+\frac{\beta\left(\frac{x_{i}}{\alpha}\right)^{-2 \beta} \log \left(\frac{x_{i}}{\alpha}\right)}{q_{i}^{2}}\right] \\
& -\frac{2 p a \beta}{\alpha} \sum_{i=1}^{n}\left[\frac{a\left(\frac{x_{i}}{\alpha}\right)^{-2 \beta} \log \left(\frac{x_{i}}{\alpha}\right) q_{i}^{-(a+2)}}{1-p\left(1-q_{i}^{-a}\right)}+\frac{\left(\frac{x_{i}}{\alpha}\right)^{-\beta} \log \left(\frac{x_{i}}{\alpha}\right) q_{i}^{-(a+1)}}{1-p\left(1-q_{i}^{-a}\right)}\right. \\
& \left.-\frac{\beta\left(\frac{x_{i}}{\alpha}\right)^{-\beta} q_{i}^{-(a+1)}}{1-p\left(1-q_{i}^{-a}\right)}-\frac{\left(\frac{x_{i}}{\alpha}\right)^{-2 \beta} \log \left(\frac{x_{i}}{\alpha}\right) q_{i}^{-(a+2)}}{1-p\left(1-q_{i}^{-a}\right)}+\frac{p a\left(\frac{x_{i}}{\alpha}\right)^{-2 \beta} \log \left(\frac{x_{i}}{\alpha}\right) q_{i}^{-2(a+1)}}{\left[1-p\left(1-q_{i}^{-a}\right)\right]^{2}}\right] \\
& \boldsymbol{J}_{\alpha p}=-\frac{2 a \beta}{\alpha} \sum_{i=1}^{n}\left[-\frac{\left(\frac{x_{i}}{\alpha}\right)^{-\beta} q_{i}^{-(a+1)}}{1-p\left(1-q_{i}^{-a}\right)}+\frac{p\left(\frac{x_{i}}{\alpha}\right)^{-\beta} q_{i}^{-(a+1)}\left(-1+q_{i}^{-a}\right)}{\left[1-p\left(1-q_{i}^{-a}\right)\right]^{2}}\right] \\
& \boldsymbol{J}_{a a}=-\frac{n}{a^{2}}-2 p \sum_{i=1}^{n}\left[\frac{\log \left(q_{i}\right)^{2} q_{i}^{-a}}{1-p\left(1-q_{i}^{-a}\right)}-\frac{p \log \left(q_{i}\right)^{2} q_{i}^{-2 a}}{\left[1-p\left(1-q_{i}^{-a}\right)\right]^{2}}\right] \\
& \boldsymbol{J}_{a \beta}=-\sum_{i=1}^{n} \frac{\left(\frac{x_{i}}{\alpha}\right)^{-\beta} \log \left(\frac{x_{i}}{\alpha}\right)}{q_{i}}-2 p \sum_{i=1}^{n}\left[-\frac{a\left(\frac{x_{i}}{\alpha}\right)^{-\beta} \log \left(\frac{x_{i}}{\alpha}\right) \log \left(q_{i}\right) q_{i}^{-(a+1)}}{1-p\left(1-q_{i}^{-a}\right)}\right. \\
& \left.+\frac{\left(\frac{x_{i}}{\alpha}\right)^{-\beta} \log \left(\frac{x_{i}}{\alpha}\right) q_{i}^{-(a+1)}}{1-p\left(1-q_{i}^{-a}\right)}+\frac{p a\left(\frac{x_{i}}{\alpha}\right)^{-\beta} \log \left(\frac{x_{i}}{\alpha}\right) \log \left(q_{i}\right) q_{i}^{-(2 a+1)}}{\left[1-p\left(1-q_{i}^{-a}\right)\right]^{2}}\right] \\
& \boldsymbol{J}_{a p}=-2 \sum_{i=1}^{n}\left[-\frac{\log \left(q_{i}\right) q_{i}^{-a}}{1-p\left(1-q_{i}^{-a}\right)}+\frac{p \log \left(q_{i}\right) q_{i}^{-a}\left(-1+q_{i}^{-a}\right)}{\left[1-p\left(1-q_{i}^{-a}\right)\right]^{2}}\right]
\end{aligned}
$$




$$
\begin{aligned}
\boldsymbol{J}_{\beta \beta}= & -\frac{n}{\beta^{2}}-(a+1) \sum_{i=1}^{n}\left[\frac{\left(\frac{x_{i}}{\alpha}\right)^{-\beta} \log \left(\frac{x_{i}}{\alpha}\right)^{2}}{q_{i}}-\frac{\left(\frac{x_{i}}{\alpha}\right)^{-2 \beta} \log \left(\frac{x_{i}}{\alpha}\right)^{2}}{q_{i}^{2}}\right] \\
& -2 p a \sum_{i=1}^{n}\left[\frac{a\left(\frac{x_{i}}{\alpha}\right)^{-2 \beta} \log \left(\frac{x_{i}}{\alpha}\right)^{2} q_{i}^{-(a+2)}}{1-p\left(1-q_{i}^{-a}\right)}-\frac{\left(\frac{x_{i}}{\alpha}\right)^{-\beta} \log \left(\frac{x_{i}}{\alpha}\right)^{2} q_{i}^{-(a+1)}}{1-p\left(1-q_{i}^{-a}\right)}\right. \\
& \left.+\frac{\left(\frac{x_{i}}{\alpha}\right)^{-2 \beta} \log \left(\frac{x_{i}}{\alpha}\right)^{2} q_{i}^{-(a+2)}}{1-p\left(1-q_{i}^{-a}\right)}-\frac{p a\left(\frac{x_{i}}{\alpha}\right)^{-2 \beta} \log \left(\frac{x_{i}}{\alpha}\right)^{2} q_{i}^{-2(a+1)}}{\left[1-p\left(1-q_{i}^{-a}\right)\right]^{2}}\right] \\
\boldsymbol{J}_{\beta p}= & -2 a \sum_{i=1}^{n}\left[\frac{\left(\frac{x_{i}}{\alpha}\right)^{-\beta} \log \left(\frac{x_{i}}{\alpha}\right) q_{i}^{-(a+1)}}{1-p\left(1-q_{i}^{-a}\right)}-\frac{p\left(\frac{x_{i}}{\alpha}\right)^{-\beta} \log \left(\frac{x_{i}}{\alpha}\right) q_{i}^{-(a+1)}\left(-1+q_{i}^{-a}\right)}{\left[1-p\left(1-q_{i}^{-a}\right)\right]^{2}}\right] \\
\boldsymbol{J}_{p p}= & \left.-\frac{n}{(1-p)^{2}}+2 \sum_{i=1}^{n} \frac{\left(-1+q_{i}^{-a}\right)^{2}}{\left[1-p\left(1-q_{i}^{-a}\right)\right]^{2}}\right] .
\end{aligned}
$$




\section{Distribuição LLEGII}

$$
\begin{aligned}
& \boldsymbol{J}_{\alpha \alpha}=-\frac{n \beta}{\alpha^{2}}-\frac{(a+1) \beta}{\alpha^{2}} \sum_{i=1}^{n}\left[\frac{\beta\left(\frac{x_{i}}{\alpha}\right)^{-\beta}}{q_{i}}-\frac{\left(\frac{x_{i}}{\alpha}\right)^{-\beta}}{q_{i}}-\frac{\beta\left(\frac{x_{i}}{\alpha}\right)^{-2 \beta}}{q_{i}^{2}}\right] \\
& -\frac{2 p a \beta}{\alpha^{2}} \sum_{i=1}^{n}\left[\frac{a \beta\left(\frac{x_{i}}{\alpha}\right)^{-2 \beta} q_{i}^{-(a+2)}}{1-p q_{i}^{-a}}+\frac{\beta\left(\frac{x_{i}}{\alpha}\right)^{-\beta} q_{i}^{-(a+1)}}{1-p q_{i}^{-a}}-\frac{\left(\frac{x_{i}}{\alpha}\right)^{-\beta} q_{i}^{-(a+1)}}{1-p q_{i}^{-a}}\right. \\
& \left.-\frac{\beta\left(\frac{x_{i}}{\alpha}\right)^{-2 \beta} q_{i}^{-(a+2)}}{1-p q_{i}^{-a}}-\frac{p a \beta\left(\frac{x_{i}}{\alpha}\right)^{-2 \beta} q_{i}^{-2(a+1)}}{\left[1-p q_{i}^{-a}\right]^{2}}\right] \\
& \boldsymbol{J}_{\alpha a}=-\frac{\beta}{\alpha} \sum_{i=1}^{n} \frac{\left(\frac{x_{i}}{\alpha}\right)^{-\beta}}{q_{i}}-\frac{2 p \beta}{\alpha} \sum_{i=1}^{n}\left[-\frac{a\left(\frac{x_{i}}{\alpha}\right)^{-\beta} \log \left(q_{i}\right) q_{i}^{-(a+1)}}{1-p q_{i}^{-a}}+\frac{\left(\frac{x_{i}}{\alpha}\right)^{-\beta} q_{i}^{-(a+1)}}{1-p q_{i}^{-a}}\right. \\
& \left.-\frac{p a \beta\left(\frac{x_{i}}{\alpha}\right)^{-\beta} \log \left(q_{i}\right) q_{i}^{-(2 a+1)}}{\left[1-p q_{i}^{-a}\right]^{2}}\right] \\
& \boldsymbol{J}_{\alpha \beta}=\frac{n}{\alpha}-\frac{(a+1)}{\alpha} \sum_{i=1}^{n}\left[-\frac{\beta\left(\frac{x_{i}}{\alpha}\right)^{-\beta} \log \left(\frac{x_{i}}{\alpha}\right)}{q_{i}}+\frac{\left(\frac{x_{i}}{\alpha}\right)^{-\beta}}{q_{i}}+\frac{\beta\left(\frac{x_{i}}{\alpha}\right)^{-2 \beta} \log \left(\frac{x_{i}}{\alpha}\right)}{q_{i}^{2}}\right] \\
& -\frac{2 p a}{\alpha} \sum_{i=1}^{n}\left[\frac{a \beta\left(\frac{x_{i}}{\alpha}\right)^{-2 \beta} \log \left(\frac{x_{i}}{\alpha}\right) q_{i}^{-(a+2)}}{1-p q_{i}^{-a}}-\frac{\beta\left(\frac{x_{i}}{\alpha}\right)^{-\beta} \log \left(\frac{x_{i}}{\alpha}\right) q_{i}^{-(a+1)}}{1-p q_{i}^{-a}}\right. \\
& \left.+\frac{\left(\frac{x_{i}}{\alpha}\right)^{-\beta} q_{i}^{-(a+1)}}{1-p q_{i}^{-a}}+\frac{\beta\left(\frac{x_{i}}{\alpha}\right)^{-2 \beta} \log \left(\frac{x_{i}}{\alpha}\right) q_{i}^{-(a+2)}}{1-p q_{i}^{-a}}+\frac{p a \beta\left(\frac{x_{i}}{\alpha}\right)^{-2 \beta} \log \left(\frac{x_{i}}{\alpha}\right) q_{i}^{-2(a+1)}}{\left[1-p q_{i}^{-a}\right]^{2}}\right] \\
& \boldsymbol{J}_{\alpha p}=-\frac{2 a \beta}{\alpha} \sum_{i=1}^{n}\left[\frac{\left(\frac{x_{i}}{\alpha}\right)^{-\beta} q_{i}^{-(a+1)}}{1-p q_{i}^{-a}}+\frac{p\left(\frac{x_{i}}{\alpha}\right)^{-\beta} q_{i}^{-(2 a+1)}}{\left[1-p q_{i}^{-a}\right]^{2}}\right] \\
& \boldsymbol{J}_{a a}=-\frac{n}{a^{2}}+2 p \sum_{i=1}^{n}\left[\frac{\log \left(q_{i}\right)^{2} q_{i}^{-a}}{1-p q_{i}^{-a}}+\frac{p \log \left(q_{i}\right)^{2} q_{i}^{-2 a}}{\left[1-p q_{i}^{-a}\right]^{2}}\right] \\
& \boldsymbol{J}_{a p}=-2 \sum_{i=1}^{n}\left[\frac{\log \left(q_{i}\right) q_{i}^{-a}}{1-p q_{i}^{-a}}+\frac{p \log \left(q_{i}\right) q_{i}^{-2 a}}{\left[1-p q_{i}^{-a}\right]^{2}}\right] \\
& \boldsymbol{J}_{a \beta}=\sum_{i=1}^{n} \frac{\left(\frac{x_{i}}{\alpha}\right)^{-\beta} \log \left(\frac{x_{i}}{\alpha}\right)}{q_{i}}-2 p \sum_{i=1}^{n}\left[\frac{a\left(\frac{x_{i}}{\alpha}\right)^{-\beta} \log \left(\frac{x_{i}}{\alpha}\right) \log \left(q_{i}\right) q_{i}^{-(a+1)}}{1-p q_{i}^{-a}}\right. \\
& \left.-\frac{\left(\frac{x_{i}}{\alpha}\right)^{-\beta} \log \left(\frac{x_{i}}{\alpha}\right) q_{i}^{-(a+1)}}{1-p q_{i}^{-a}}+\frac{p a\left(\frac{x_{i}}{\alpha}\right)^{-\beta} \log \left(\frac{x_{i}}{\alpha}\right) \log \left(q_{i}\right) q_{i}^{-(2 a+1)}}{\left[1-p q_{i}^{-a}\right]^{2}}\right] \\
& \boldsymbol{J}_{\beta \beta}=-\frac{n}{\beta^{2}}-(a+1) \sum_{i=1}^{n}\left[\frac{\left(\frac{x_{i}}{\alpha}\right)^{-\beta} \log \left(\frac{x_{i}}{\alpha}\right)^{2}}{q_{i}}-\frac{\left(\frac{x_{i}}{\alpha}\right)^{-2 \beta} \log \left(\frac{x_{i}}{\alpha}\right)^{2}}{q_{i}^{2}}\right] \\
& -2 p a \sum_{i=1}^{n}\left[-\frac{a\left(\frac{x_{i}}{\alpha}\right)^{-2 \beta} \log \left(\frac{x_{i}}{\alpha}\right)^{2} q_{i}^{-(a+2)}}{1-p q_{i}^{-a}}+\frac{\left(\frac{x_{i}}{\alpha}\right)^{-\beta} \log \left(\frac{x_{i}}{\alpha}\right)^{2} q_{i}^{-(a+1)}}{1-p q_{i}^{-a}}\right. \\
& \left.-\frac{\left(\frac{x_{i}}{\alpha}\right)^{-2 \beta} \log \left(\frac{x_{i}}{\alpha}\right)^{2} q_{i}^{-(a+2)}}{1-p q_{i}^{-a}}-\frac{p a\left(\frac{x_{i}}{\alpha}\right)^{-2 \beta} \log \left(\frac{x_{i}}{\alpha}\right)^{2} q_{i}^{-2(a+1)}}{\left[1-p q_{i}^{-a}\right]^{2}}\right] \\
& \boldsymbol{J}_{\beta p}=2 a \sum_{i=1}^{n}\left[\frac{\left(\frac{x_{i}}{\alpha}\right)^{-\beta} \log \left(\frac{x_{i}}{\alpha}\right) q_{i}^{-(a+1)}}{1-p q_{i}^{-a}}+\frac{p\left(\frac{x_{i}}{\alpha}\right)^{-\beta} \log \left(\frac{x_{i}}{\alpha}\right) q_{i}^{-(2 a+1)}}{\left[1-p q_{i}^{-a}\right]^{2}}\right] \\
& \boldsymbol{J}_{p p}=-\frac{n}{(1-p)^{2}}+2 \sum_{i=1}^{n} \frac{q_{i}^{-2 a}}{\left[1-p q_{i}^{-a}\right]^{2}} \text {. }
\end{aligned}
$$




\section{APÊNDICE B - Modelo de regressão}

\section{B.1 Componentes do vetor escore}

Os estimadores de máxima verossimilhança para os parâmetros $a, p, \sigma$ e $\beta_{k}$, para $k=1, \ldots, q$, são obtidos do sistema de equações de

\section{Modelo LEGI}

$$
\begin{aligned}
& \frac{\partial l(\boldsymbol{\theta})}{\partial a}=\frac{r}{a}+\sum_{i \in F}\left(\frac{y_{i}-\mathbf{v}_{i}^{T} \beta}{\sigma}\right)-\sum_{i \in F} \log \left[1+\exp \left(\frac{y_{i}-\mathbf{v}_{i}^{T} \beta}{\sigma}\right)\right]+2 p \sum_{i \in F} \frac{q_{i}^{-a} \log \left(q_{i}\right)}{1-p\left(1-q_{i}^{-a}\right)} \\
& +\sum_{i \in C}\left[\frac{q_{i}^{-a} \log \left(q_{i}\right)}{1-p\left(1-q_{i}^{-a}\right)}-\frac{p q_{i}^{-2 a} \log \left(q_{i}\right)}{\left[1-p\left(1-q_{i}^{-a}\right)\right]^{2}}\right] \\
& \times\left\{1-q_{i}^{-a}\left[1-p\left(1-q_{i}^{-a}\right)\right]^{-1}\right\}^{-1}=0 \\
& \frac{\partial l(\boldsymbol{\theta})}{\partial p}=-\frac{r}{1-p}-2 \sum_{i \in F} \frac{\left(-1+q_{i}^{-a}\right)}{1-p\left(1-q_{i}^{-a}\right)}+\sum_{i \in C}\left\{q_{i}^{-a}\left(-1+q_{i}^{-a}\right)\left[1-p\left(1-q_{i}^{-a}\right)\right]^{-2}\right\} \times \\
& \left\{1-q_{i}^{-a}\left[1-p\left(1-q_{i}^{-a}\right)\right]^{-1}\right\}^{-1}=0 \\
& \frac{\partial l(\boldsymbol{\theta})}{\partial \sigma}=-\frac{r}{\sigma}-a \sum_{i \in F}\left(\frac{y_{i}-\mathbf{v}_{i}^{T} \beta}{\sigma^{2}}\right)+(a+1) \sum_{i \in F} \frac{\left(\frac{y_{i}-\mathbf{v}_{i}^{T} \beta}{\sigma^{2}}\right) \exp \left(\frac{y_{i}-\mathbf{v}_{i}^{T} \beta}{\sigma}\right)}{\left[1+\exp \left(\frac{y_{i}-\mathbf{v}_{i}^{T} \beta}{\sigma}\right)\right]} \\
& +2 a p \sum_{i \in F}\left(\frac{y_{i}-\mathbf{v}_{i}^{T} \beta}{\sigma^{2}}\right) \exp \left[-\left(\frac{y_{i}-\mathbf{v}_{i}^{T} \beta}{\sigma}\right)\right] q_{i}^{-(a+1)}\left[1-p\left(1-q_{i}^{-a}\right)\right]^{-1}+ \\
& \sum_{i \in C}\left\{\frac{a\left(\frac{y_{i}-\mathbf{v}_{i}^{T} \beta}{\sigma^{2}}\right) \exp \left[-\left(\frac{y_{i}-\mathbf{v}_{i}^{T} \beta}{\sigma}\right)\right] q_{i}^{-(a+1)}}{1-p\left(1-q_{i}^{-a}\right)}\right. \\
& \left.-\frac{a p\left(\frac{y_{i}-\mathbf{v}_{i}^{T} \beta}{\sigma^{2}}\right) \exp \left[-\left(\frac{y_{i}-\mathbf{v}_{i}^{T} \beta}{\sigma}\right)\right] q_{i}^{-2 a-1}}{\left[1-p\left(1-q_{i}^{-a}\right)\right]^{2}}\right\} \\
& \times\left\{1-q_{i}^{-a}\left[1-p\left(1-q_{i}^{-a}\right)\right]^{-1}\right\}^{-1}=0 \\
& \frac{\partial l(\boldsymbol{\theta})}{\partial \beta_{k}}=-\frac{a}{\sigma} \sum_{i \in F} \mathrm{v}_{i k}+\frac{(a+1)}{\sigma} \sum_{i \in F} \frac{\exp \left(\frac{y_{i}-\mathbf{v}_{i}^{T} \beta}{\sigma}\right) \mathrm{v}_{i k}}{1+\exp \left(\frac{y_{i}-\mathbf{v}_{i}^{T} \beta}{\sigma}\right)}+\frac{2 p a}{\sigma} \sum_{i \in F} \frac{\exp \left[-\left(\frac{y_{i}-\mathbf{v}_{i}^{T} \beta}{\sigma}\right)\right] q_{i}^{-(a+1)} \mathrm{v}_{i k}}{1-p\left(1-q_{i}^{-a}\right)} \\
& -\sum_{i \in C}\left\{\frac{\exp \left[-\left(\frac{y_{i}-\mathbf{v}_{i}^{T} \beta}{\sigma}\right)\right] \mathrm{v}_{i k}}{\sigma\left[1-q_{i}^{-a}\left[1-p\left(1-q_{i}^{-a}\right)\right]^{-1}\right]}\left[\frac{p a q_{i}^{-2 a-1}}{\left[1-p\left(1-q_{i}^{-a}\right)\right]^{2}}+\frac{1}{\left[1-p\left(1-q_{i}^{-a}\right)\right]}\right]\right\}=0
\end{aligned}
$$




\section{Modelo LEGII}

$$
\begin{aligned}
& \frac{\partial l(\boldsymbol{\theta})}{\partial a}=\frac{r}{a}+\sum_{i \in F}\left(\frac{y_{i}-\mathbf{v}_{i}^{T} \beta}{\sigma}\right)-\sum_{i \in F} \log \left[1+\exp \left(\frac{y_{i}-\mathbf{v}_{i}^{T} \beta}{\sigma}\right)\right] \\
& -2 p \sum_{i \in F} \frac{q_{i}^{-a} \log \left(q_{i}\right)}{1-p q_{i}^{-a}}+\sum_{i \in C}\left\{\frac{(1-p) q_{i}^{-a} \log \left(q_{i}\right)}{1-p q_{i}^{-a}}+\frac{(1-p) p q_{i}^{-2 a} \log \left(q_{i}\right)}{\left[1-p q_{i}^{-a}\right]^{2}}\right\} \\
& \times\left\{1-(1-p) q_{i}^{-a}\left[1-p q_{i}^{-a}\right]^{-1}\right\}^{-1}=0 \\
& \frac{\partial l(\boldsymbol{\theta})}{\partial p}=-\frac{r}{1-p}+2 \sum_{i \in F} \frac{q_{i}^{-a}}{1-p q_{i}^{-a}}+\sum_{i \in C}\left\{\frac{q_{i}^{-a}}{1-p q_{i}^{-a}}-\frac{(1-p) q_{i}^{-2 a}}{\left[1-p q_{i}^{-a}\right]^{2}}\right\} \times \\
& \left\{1-(1-p) q_{i}^{-a}\left[1-p q_{i}^{-a}\right]^{-1}\right\}^{-1}=0 \\
& \frac{\partial l(\boldsymbol{\theta})}{\partial \sigma}=-\frac{r}{\sigma}-a \sum_{i \in F}\left(\frac{y_{i}-\mathbf{v}_{i}^{T} \beta}{\sigma^{2}}\right)+(a+1) \sum_{i \in F} \frac{\left(\frac{y_{i}-\mathbf{v}_{i}^{T} \beta}{\sigma^{2}}\right) \exp \left(\frac{y_{i}-\mathbf{v}_{i}^{T} \beta}{\sigma}\right)}{\left[1+\exp \left(\frac{y_{i}-\mathbf{v}_{i}^{T} \beta}{\sigma}\right)\right]} \\
& -2 a p \sum_{i \in F}\left(\frac{y_{i}-\mathbf{v}_{i}^{T} \beta}{\sigma^{2}}\right) \exp \left[-\left(\frac{y_{i}-\mathbf{v}_{i}^{T} \beta}{\sigma}\right)\right] q_{i}^{-(a+1)}\left[1-p q_{i}^{-a}\right]^{-1} \\
& +\sum_{i \in C}\left\{\frac{a(1-p)\left(\frac{y_{i}-\mathbf{v}_{i}^{T} \beta}{\sigma^{2}}\right) \exp \left[-\left(\frac{y_{i}-\mathbf{v}_{i}^{T} \beta}{\sigma}\right)\right] q_{i}^{-(a+1)}}{1-p q_{i}^{-a}}\right. \\
& \left.+\frac{a p(1-p)\left(\frac{y_{i}-\mathbf{v}_{i}^{T} \beta}{\sigma^{2}}\right) \exp \left[-\left(\frac{y_{i}-\mathbf{v}_{i}^{T} \beta}{\sigma}\right)\right] q_{i}^{-2 a-1}}{\left[1-p q_{i}^{-a}\right]^{2}}\right\} \\
& \times\left\{1-(1-p) q_{i}^{-a}\left[1-p q_{i}^{-a}\right]^{-1}\right\}^{-1}=0 \\
& \frac{\partial l(\boldsymbol{\theta})}{\partial \beta_{k}}=-\frac{a}{\sigma} \sum_{i \in F} \mathrm{v}_{i k}+\frac{(a+1)}{\sigma} \sum_{i \in F} \frac{\exp \left(\frac{y_{i}-\mathbf{v}_{i}^{T} \beta}{\sigma}\right) \mathrm{v}_{i k}}{1+\exp \left(\frac{y_{i}-\mathbf{v}_{i}^{T} \beta}{\sigma}\right)} \\
& -\frac{2 p a}{\sigma} \sum_{i \in F} \frac{\exp \left[-\left(\frac{y_{i}-\mathbf{v}_{i}^{T} \beta}{\sigma}\right)\right] q_{i}^{-(a+1)} \mathrm{v}_{i k}}{1-p q_{i}^{-a}} \\
& -\sum_{i \in C}\left\{\frac{\exp \left[-\left(\frac{y_{i}-\mathbf{v}_{i}^{T} \beta}{\sigma}\right)\right] \mathrm{v}_{i k}}{\sigma\left[1-(1-p) q_{i}^{-a}\left[1-p q_{i}^{-a}\right]^{-1}\right]}\right. \\
& \left.\times\left[-\frac{(1-p) p a q_{i}^{-2 a-1}}{\left[1-p q_{i}^{-a}\right]^{2}}+\frac{(1-p)}{\left[1-p q_{i}^{-a}\right]}\right]\right\}=0
\end{aligned}
$$

em que, $q_{i}=1+\exp \left[-\left(\frac{y_{i}-\mathbf{v}_{i}^{T} \beta}{\sigma}\right)\right]$.

B.2 Matriz de informação observada $\boldsymbol{J}(\boldsymbol{\theta})$ : As derivadas da segunda ordem do logaritmo da função de verossimilhança, dos modelos LEGI e LEGII são dadas a seguir, considerando a seguinte expressão, $q_{i}=1+\exp \left[-\left(\frac{y_{i}-\mathbf{v}_{i}^{T} \beta}{\sigma}\right)\right]$ 


\section{Modelo LEGI}

$$
\begin{aligned}
& \boldsymbol{J}_{a a}=-\frac{r}{\alpha^{2}}-2 p \sum_{i \in F}\left[\frac{\log \left(q_{i}\right)^{2} q_{i}^{-a}}{1-p\left(1-q_{i}^{-a}\right)}-\frac{p \log \left(q_{i}\right)^{2} q_{i}^{-2 a}}{\left[1-p\left(1-q_{i}^{-a}\right)\right]^{2}}\right]+\sum_{i \in C}\left\{\frac{1-p\left(1-q_{i}^{-a}\right)}{1-p\left(1-q_{i}^{-a}\right)-q_{i}^{-a}}\right. \\
& \times\left[-\frac{\log \left(q_{i}\right)^{2} q_{i}^{-a}}{1-p\left(1-q_{i}^{-a}\right)}+\frac{3 p \log \left(q_{i}\right)^{2} q_{i}^{-2 a}}{\left[1-p\left(1-q_{i}^{-a}\right)\right]^{2}}-\frac{2 p^{2} \log \left(q_{i}\right)^{2} q_{i}^{-3 a}}{\left[1-p\left(1-q_{i}^{-a}\right)\right]^{3}}\right] \\
& \left.-\frac{\left[1-p\left(1-q_{i}^{-a}\right)\right]^{2}}{\left[1-p\left(1-q_{i}^{-a}\right)-q_{i}^{-a}\right]^{2}}\left[\frac{\log \left(q_{i}\right) q_{i}^{-a}}{1-p\left(1-q_{i}^{-a}\right)}-\frac{p \log \left(q_{i}\right) q_{i}^{-2 a}}{\left[1-p\left(1-q_{i}^{-a}\right)\right]^{2}}\right]^{2}\right\} \\
& \boldsymbol{J}_{a \sigma}=\sum_{i \in F}\left[-\left(\frac{y_{i}-\mathbf{v}_{i}^{T} \beta}{\sigma^{2}}\right)\right]+\sum_{i \in F} \frac{\left(\frac{y_{i}-\mathbf{v}_{i}^{T} \beta}{\sigma^{2}}\right) \exp \left(\frac{y_{i}-\mathbf{v}_{i}^{T} \beta}{\sigma^{2}}\right)}{1+\exp \left(\frac{y_{i}-\mathbf{v}_{i}^{T} \beta}{\sigma}\right)} \\
& -2 p \sum_{i \in F}\left[\frac{a\left(\frac{y_{i}-\mathbf{v}_{i}^{T} \beta}{\sigma^{2}}\right) \exp \left[-\left(\frac{y_{i}-\mathbf{v}_{i}^{T} \beta}{\sigma}\right)\right] \log \left(q_{i}\right) q_{i}^{-(a+1)}}{1-p\left(1-q_{i}^{-a}\right)}\right. \\
& \left.-\frac{\left(\frac{y_{i}-\mathbf{v}_{i}^{T} \beta}{\sigma^{2}}\right) \exp \left[-\left(\frac{y_{i}-\mathbf{v}_{i}^{T} \beta}{\sigma}\right)\right] q_{i}^{-(a+1)}}{1-p\left(1-q_{i}^{-a}\right)}-\frac{p a\left(\frac{y_{i}-\mathbf{v}_{i}^{T} \beta}{\sigma^{2}}\right) \exp \left[-\left(\frac{y_{i}-\mathbf{v}_{i}^{T} \beta}{\sigma}\right)\right] \log \left(q_{i}\right) q_{i}^{-(2 a+1)}}{\left[1-p\left(1-q_{i}^{-a}\right)\right]^{2}}\right] \\
& +\sum_{i \in C}\left\{\frac { 1 - p ( 1 - q _ { i } ^ { - a } ) } { 1 - p ( 1 - q _ { i } ^ { - a } ) - q _ { i } ^ { - a } } \left[-\frac{a\left(\frac{y_{i}-\mathbf{v}_{i}^{T} \beta}{\sigma^{2}}\right) \exp \left[-\left(\frac{y_{i}-\mathbf{v}_{i}^{T} \beta}{\sigma}\right)\right] \log \left(q_{i}\right) q_{i}^{-(a+1)}}{1-p\left(1-q_{i}^{-a}\right)}\right.\right. \\
& +\frac{\left(\frac{y_{i}-\mathbf{v}_{i}^{T} \beta}{\sigma^{2}}\right) \exp \left[-\left(\frac{y_{i}-\mathbf{v}_{i}^{T} \beta}{\sigma}\right)\right] q_{i}^{-(a+1)}}{1-p\left(1-q_{i}^{-a}\right)} \\
& +\frac{3 p a\left(\frac{y_{i}-\mathbf{v}_{i}^{T} \beta}{\sigma^{2}}\right) \exp \left[-\left(\frac{y_{i}-\mathbf{v}_{i}^{T} \beta}{\sigma}\right)\right] \log \left(q_{i}\right) q_{i}^{-(2 a+1)}}{\left[1-p\left(1-q_{i}^{-a}\right)\right]^{2}} \\
& -\frac{2 p^{2} a\left(\frac{y_{i}-\mathbf{v}_{i}^{T} \beta}{\sigma^{2}}\right) \exp \left[-\left(\frac{y_{i}-\mathbf{v}_{i}^{T} \beta}{\sigma}\right)\right] \log \left(q_{i}\right) q_{i}^{-(3 a+1)}}{\left[1-p\left(1-q_{i}^{-a}\right)\right]^{3}} \\
& \left.-\frac{p\left(\frac{y_{i}-\mathbf{v}_{i}^{T} \beta}{\sigma^{2}}\right) \exp \left[-\left(\frac{y_{i}-\mathbf{v}_{i}^{T} \beta}{\sigma}\right)\right] q_{i}^{-(2 a+1)}}{\left[1-p\left(1-q_{i}^{-a}\right)\right]^{2}}\right] \\
& -\frac{\left[1-p\left(1-q_{i}^{-a}\right)\right]^{2}}{\left[1-p\left(1-q_{i}^{-a}\right)-q_{i}^{-a}\right]^{2}}\left[\frac{a\left(\frac{y_{i}-\mathbf{v}_{i}^{T} \beta}{\sigma^{2}}\right) \exp \left[-\left(\frac{y_{i}-\mathbf{v}_{i}^{T} \beta}{\sigma}\right)\right] q_{i}^{-(a+1)}}{1-p\left(1-q_{i}^{-a}\right)}\right. \\
& \left.\left.-\frac{p a\left(\frac{y_{i}-\mathbf{v}_{i}^{T} \beta}{\sigma^{2}}\right) \exp \left[-\left(\frac{y_{i}-\mathbf{v}_{i}^{T} \beta}{\sigma}\right)\right] q_{i}^{-(2 a+1)}}{\left[1-p\left(1-q_{i}^{-a}\right)\right]^{2}}\right]\left[\frac{\log \left(q_{i}\right) q_{i}^{-a}}{1-p\left(1-q_{i}^{-a}\right)}-\frac{p \log \left(q_{i}\right) q_{i}^{-2 a}}{\left[1-p\left(1-q_{i}^{-a}\right)\right]^{2}}\right]\right\} \\
& \boldsymbol{J}_{a p}=-2 \sum_{i \in F}\left[-\frac{\log \left(q_{i}\right) q_{i}^{-a}}{1-p\left(1-q_{i}^{-a}\right)}+\frac{p \log \left(q_{i}\right) q_{i}^{-a}\left(-1+q_{i}^{-a}\right)}{\left[1-p\left(1-q_{i}^{-a}\right)\right]^{2}}\right] \\
& +\sum_{i \in C}\left\{\frac { 1 - p ( 1 - q _ { i } ^ { - a } ) } { 1 - p ( 1 - q _ { i } ^ { - a } ) - q _ { i } ^ { - a } } \left[-\frac{\log \left(q_{i}\right) q_{i}^{-a}\left(-1+q_{i}^{-a}\right)}{\left[1-p\left(1-q_{i}^{-a}\right)\right]^{2}}+\frac{2 p \log \left(q_{i}\right) q_{i}^{-2 a}\left(-1+q_{i}^{-a}\right)}{\left[1-p\left(1-q_{i}^{-a}\right)\right]^{3}}\right.\right. \\
& \left.\left.-\frac{\log \left(q_{i}\right) q_{i}^{-2 a}}{\left[1-p\left(1-q_{i}^{-a}\right)\right]^{2}}\right]-\left[\frac{q_{i}^{-a}\left(-1+q_{i}^{-a}\right)}{\left[1-p\left(1-q_{i}^{-a}\right)\right]^{2}}\left[\frac{q_{i}^{-a} \log \left(q_{i}\right)}{1-p\left(1-q_{i}^{-a}\right)}-\frac{p q_{i}^{-2 a} \log \left(q_{i}\right)}{\left[1-p\left(1-q_{i}^{-a}\right)\right]^{2}}\right]\right]\right\}
\end{aligned}
$$




$$
\begin{aligned}
& \boldsymbol{J}_{\sigma \sigma}=\frac{r}{\sigma^{2}}+2 a \sum_{i \in F}\left(\frac{y_{i}-\mathbf{v}_{i}^{T} \beta}{\sigma^{3}}\right)-(a+1) \sum_{i \in F}\left[\frac{2\left(\frac{y_{i}-\mathbf{v}_{i}^{T} \beta}{\sigma^{3}}\right) \exp \left(\frac{y_{i}-\mathbf{v}_{i}^{T} \beta}{\sigma}\right)}{1+\exp \left(\frac{y_{i}-\mathbf{v}_{i}^{T} \beta}{\sigma}\right)}\right. \\
& \left.+\frac{\left(\frac{y_{i}-\mathbf{v}_{i}^{T} \beta}{\sigma^{2}}\right)^{2} \exp \left(\frac{y_{i}-\mathbf{v}_{i}^{T} \beta}{\sigma}\right)}{1+\exp \left(\frac{y_{i}-\mathbf{v}_{i}^{T} \beta}{\sigma}\right)}-\frac{\left(\frac{y_{i}-\mathbf{v}_{i}^{T} \beta}{\sigma^{2}}\right)^{2}\left[\exp \left(\frac{y_{i}-\mathbf{v}_{i}^{T} \beta}{\sigma}\right)\right]^{2}}{\left[1+\exp \left(\frac{y_{i}-\mathbf{v}_{i}^{T} \beta}{\sigma}\right)\right]^{2}}\right] \\
& -2 p a \sum_{i \in F}\left[\frac{a\left(\frac{y_{i}-\mathbf{v}_{i}^{T} \beta}{\sigma^{2}}\right)^{2}\left[\exp \left(-\frac{y_{i}-\mathbf{v}_{i}^{T} \beta}{\sigma}\right)\right]^{2} q_{i}^{-(a+2)}}{1-p\left(1-q_{i}^{-a}\right)}\right. \\
& +\frac{\left(\frac{y_{i}-\mathbf{v}_{i}^{T} \beta}{\sigma^{3}}\right)\left[\exp \left(-\frac{y_{i}-\mathbf{v}_{i}^{T} \beta}{\sigma}\right)\right] q_{i}^{-(a+1)}}{1-p\left(1-q_{i}^{-a}\right)}-\frac{\left(\frac{y_{i}-\mathbf{v}_{i}^{T} \beta}{\sigma^{2}}\right)^{2}\left[\exp \left(-\frac{y_{i}-\mathbf{v}_{i}^{T} \beta}{\sigma}\right)\right] q_{i}^{-(a+1)}}{1-p\left(1-q_{i}^{-a}\right)} \\
& \left.+\frac{\left(\frac{y_{i}-\mathbf{v}_{i}^{T} \beta}{\sigma^{2}}\right)^{2}\left[\exp \left(-\frac{y_{i}-\mathbf{v}_{i}^{T} \beta}{\sigma}\right)\right]^{2} q_{i}^{-(a+2)}}{1-p\left(1-q_{i}^{-a}\right)}-\frac{p a\left(\frac{y_{i}-\mathbf{v}_{i}^{T} \beta}{\sigma^{2}}\right)^{2}\left[\exp \left(-\frac{y_{i}-\mathbf{v}_{i}^{T} \beta}{\sigma}\right)\right]^{2} q_{i}^{-2(a+1)}}{\left[1-p\left(1-q_{i}^{-a}\right)\right]^{2}}\right] \\
& +\sum_{i \in C}\left\{\frac { 1 - p ( 1 - q _ { i } ^ { - a } ) } { 1 - p ( 1 - q _ { i } ^ { - a } ) - q _ { i } ^ { - a } } \left[-\frac{a^{2}\left(\frac{y_{i}-\mathbf{v}_{i}^{T} \beta}{\sigma^{2}}\right)^{2}\left[\exp \left(-\frac{y_{i}-\mathbf{v}_{i}^{T} \beta}{\sigma}\right)\right]^{2} q_{i}^{-(a+2)}}{1-p\left(1-q_{i}^{-a}\right)}\right.\right. \\
& -\frac{2 a\left(\frac{y_{i}-\mathbf{v}_{i}^{T} \beta}{\sigma^{3}}\right)\left[\exp \left(-\frac{y_{i}-\mathbf{v}_{i}^{T} \beta}{\sigma}\right)\right] q_{i}^{-(a+1)}}{1-p\left(1-q_{i}^{-a}\right)}+\frac{a\left(\frac{y_{i}-\mathbf{v}_{i}^{T} \beta}{\sigma^{2}}\right)^{2}\left[\exp \left(-\frac{y_{i}-\mathbf{v}_{i}^{T} \beta}{\sigma}\right)\right] q_{i}^{-(a+1)}}{1-p\left(1-q_{i}^{-a}\right)} \\
& -\frac{a\left(\frac{y_{i}-\mathbf{v}_{i}^{T} \beta}{\sigma^{2}}\right)^{2}\left[\exp \left(-\frac{y_{i}-\mathbf{v}_{i}^{T} \beta}{\sigma}\right)\right]^{2} q_{i}^{-(a+2)}}{1-p\left(1-q_{i}^{-a}\right)}+\frac{3 p a^{2}\left(\frac{y_{i}-\mathbf{v}_{i}^{T} \beta}{\sigma^{2}}\right)^{2}\left[\exp \left(-\frac{y_{i}-\mathbf{v}_{i}^{T} \beta}{\sigma}\right)\right]^{2} q_{i}^{-2(a+1)}}{\left[1-p\left(1-q_{i}^{-a}\right)\right]^{2}} \\
& -\frac{2 p^{2} a^{2}\left(\frac{y_{i}-\mathbf{v}_{i}^{T} \beta}{\sigma^{2}}\right)^{2}\left[\exp \left(-\frac{y_{i}-\mathbf{v}_{i}^{T} \beta}{\sigma}\right)\right]^{2} q_{i}^{-(3 a+2)}}{\left[1-p\left(1-q_{i}^{-a}\right)\right]^{3}}+\frac{2 p a\left(\frac{y_{i}-\mathbf{v}_{i}^{T} \beta}{\sigma^{3}}\right)\left[\exp \left(-\frac{y_{i}-\mathbf{v}_{i}^{T} \beta}{\sigma}\right)\right] q_{i}^{-(2 a+1)}}{\left[1-p\left(1-q_{i}^{-a}\right)\right]^{2}} \\
& \left.-\frac{p a\left(\frac{y_{i}-\mathbf{v}_{i}^{T} \beta}{\sigma^{2}}\right)^{2}\left[\exp \left(-\frac{y_{i}-\mathbf{v}_{i}^{T} \beta}{\sigma}\right)\right] q_{i}^{-(2 a+1)}}{\left[1-p\left(1-q_{i}^{-a}\right)\right]^{2}}+\frac{p a\left(\frac{y_{i}-\mathbf{v}_{i}^{T} \beta}{\sigma^{2}}\right)^{2}\left[\exp \left(-\frac{y_{i}-\mathbf{v}_{i}^{T} \beta}{\sigma}\right)\right]^{2} q_{i}^{-2(a+1)}}{\left[1-p\left(1-q_{i}^{-a}\right)\right]^{2}}\right] \\
& -\frac{\left[1-p\left(1-q_{i}^{-a}\right)\right]^{2}}{\left[1-p\left(1-q_{i}^{-a}\right)-q_{i}^{-a}\right]^{2}}\left[\frac{a\left(\frac{y_{i}-\mathbf{v}_{i}^{T} \beta}{\sigma^{2}}\right)\left[\exp \left(-\frac{y_{i}-\mathbf{v}_{i}^{T} \beta}{\sigma}\right)\right] q_{i}^{-(a+1)}}{1-p\left(1-q_{i}^{-a}\right)}\right. \\
& \left.\left.-\frac{p a\left(\frac{y_{i}-\mathbf{v}_{i}^{T} \beta}{\sigma^{2}}\right)\left[\exp \left(-\frac{y_{i}-\mathbf{v}_{i}^{T} \beta}{\sigma}\right)\right] q_{i}^{-(2 a+1)}}{\left[1-p\left(1-q_{i}^{-a}\right)\right]^{2}}\right]^{2}\right\} \\
& \boldsymbol{J}_{\sigma p}=-2 a \sum_{i \in F}\left[-\frac{\left(\frac{y_{i}-\mathbf{v}_{i}^{T} \beta}{\sigma^{2}}\right)\left[\exp \left(-\frac{y_{i}-\mathbf{v}_{i}^{T} \beta}{\sigma}\right)\right] q_{i}^{-(a+1)}}{1-p\left(1-q_{i}^{-a}\right)}\right.
\end{aligned}
$$$$
\left.+\frac{p\left(\frac{y_{i}-\mathbf{v}_{i}^{T} \beta}{\sigma^{2}}\right) \exp \left(-\frac{y_{i}-\mathbf{v}_{i}^{T} \beta}{\sigma}\right) q_{i}^{-(a+1)}\left(-1+q_{i}^{-a}\right)}{1-p\left(1-q_{i}^{-a}\right)}\right]
$$$$
+\sum_{i \in C}\left\{\frac { 1 - p ( 1 - q _ { i } ^ { - a } ) } { 1 - p ( 1 - q _ { i } ^ { - a } ) - q _ { i } ^ { - a } } \left[-\frac{a\left(\frac{y_{i}-\mathbf{v}_{i}^{T} \beta}{\sigma^{2}}\right)\left[\exp \left(-\frac{y_{i}-\mathbf{v}_{i}^{T} \beta}{\sigma}\right)\right] q_{i}^{-(a+1)}\left(-1+q_{i}^{-a}\right)}{\left[1-p\left(1-q_{i}^{-a}\right)\right]^{2}}\right.\right.
$$ 


$$
\begin{aligned}
& +\frac{2 p a\left(\frac{y_{i}-\mathbf{v}_{i}^{T} \beta}{\sigma^{2}}\right)\left[\exp \left(-\frac{y_{i}-\mathbf{v}_{i}^{T} \beta}{\sigma}\right)\right] q_{i}^{-(2 a+1)}\left(-1+q_{i}^{-a}\right)}{\left[1-p\left(1-q_{i}^{-a}\right)\right]^{3}} \\
& \left.-\frac{a\left(\frac{y_{i}-\mathbf{v}_{i}^{T} \beta}{\sigma^{2}}\right)\left[\exp \left(-\frac{y_{i}-\mathbf{v}_{i}^{T} \beta}{\sigma}\right)\right] q_{i}^{-(2 a+1)}}{\left[1-p\left(1-q_{i}^{-a}\right)\right]^{2}}\right] \\
& -\frac{1}{1-p\left(1-q_{i}^{-a}\right)-q_{i}^{-a}}\left[\frac{a\left(\frac{y_{i}-\mathbf{v}_{i}^{T} \beta}{\sigma^{2}}\right)\left[\exp \left(-\frac{y_{i}-\mathbf{v}_{i}^{T} \beta}{\sigma}\right)\right] q_{i}^{-(2 a+1)}\left(-1+q_{i}^{-a}\right)}{\left[1-p\left(1-q_{i}^{-a}\right)\right]}\right. \\
& \left.\left.-\frac{p a\left(\frac{y_{i}-\mathbf{v}_{i}^{T} \beta}{\sigma^{2}}\right)\left[\exp \left(-\frac{y_{i}-\mathbf{v}_{i}^{T} \beta}{\sigma}\right)\right] q_{i}^{-(3 a+1)}\left(-1+q_{i}^{-a}\right)}{\left[1-p\left(1-q_{i}^{-a}\right)\right]^{2}}\right]\right\} \\
& \boldsymbol{J}_{p p}=-\frac{r}{(1-p)^{2}}+2 \sum_{i \in F} \frac{\left(-1+q_{i}^{-a}\right)^{2}}{\left[1-p\left(1-q_{i}^{-a}\right)\right]^{2}} \\
& +\sum_{i \in C}\left[-\frac{2 q_{i}^{-a}\left(-1+q_{i}^{-a}\right)^{2}\left[1-p\left(1-q_{i}^{-a}\right)\right]}{\left[1-p\left(1-q_{i}^{-a}\right)\right]^{3}\left[1-p\left(1-q_{i}^{-a}\right)-q_{i}^{-a}\right]}\right. \\
& \left.-\frac{q_{i}^{-2 a}\left(-1+q_{i}^{-a}\right)^{2}\left[1-p\left(1-q_{i}^{-a}\right)\right]^{2}}{\left[1-p\left(1-q_{i}^{-a}\right)\right]^{4}\left[1-p\left(1-q_{i}^{-a}\right)-q_{i}^{-a}\right]^{2}}\right] \\
& \boldsymbol{J}_{a \beta_{k}}=-\frac{1}{\sigma} \sum_{i \in F} \mathrm{v}_{i k}+\frac{1}{\sigma} \sum_{i \in F} \frac{\exp \left(\frac{y_{i}-\mathbf{v}_{i}^{T} \beta}{\sigma}\right) \mathrm{v}_{i k}}{1+\exp \left(\frac{y_{i}-\mathbf{v}_{i}^{T} \beta}{\sigma}\right)}+\frac{2 p}{\sigma} \sum_{i \in F}\left\{\frac{\exp \left[-\left(\frac{y_{i}-\mathbf{v}_{i}^{T} \beta}{\sigma}\right)\right] q_{i}^{-(a+1)} \mathrm{v}_{i k}}{1-p\left(1-q_{i}^{-a}\right)}\right. \\
& \left.-\frac{a \exp \left[-\left(\frac{y_{i}-\mathbf{v}_{i}^{T} \beta}{\sigma}\right)\right] q_{i}^{-(a+1)} \ln \left(q_{i}\right) \mathrm{v}_{i k}}{\left[1-p\left(1-q_{i}^{-a}\right)\right]}+\frac{p a \exp \left[-\left(\frac{y_{i}-\mathbf{v}_{i}^{T} \beta}{\sigma}\right)\right] q_{i}^{-2 a-1} \ln \left(q_{i}\right) \mathrm{v}_{i k}}{\left[1-p\left(1-q_{i}^{-a}\right)\right]^{2}}\right\} \\
& -\frac{1}{\sigma} \sum_{i \in C}\left\{-\exp \left[-\left(\frac{y_{i}-\mathbf{v}_{i}^{T} \beta}{\sigma}\right)\right] \mathrm{v}_{i k}\left[\frac{p a q_{i}^{-2 a-1}}{\left[1-p\left(1-q_{i}^{-a}\right)\right]^{2}}+\frac{1}{1-p\left(1-q_{i}^{-a}\right)}\right]\right. \\
& \times\left[\frac{q_{i}^{-a} \ln \left(q_{i}\right)}{1-p\left(1-q_{i}^{-a}\right)}-\frac{p q_{i}^{-2 a} \ln \left(q_{i}\right)}{\left[1-p\left(1-q_{i}^{-a}\right)\right]^{2}}\right]\left[1-\frac{q_{i}^{-a}}{1-p\left(1-q_{i}^{-a}\right)}\right]^{-2} \\
& +\exp \left[-\left(\frac{y_{i}-\mathbf{v}_{i}^{T} \beta}{\sigma}\right)\right] \mathrm{v}_{i k}\left[\frac{p q_{i}^{-2 a-1}}{\left[1-p\left(1-q_{i}^{-a}\right)\right]^{2}}-\frac{2 p a q_{i}^{-2 a-1} \ln \left(q_{i}\right)}{\left[1-p\left(1-q_{i}^{-a}\right)\right]^{2}}\right. \\
& \left.\left.+\frac{2 p^{2} a q_{i}^{-3 a-1} \ln \left(q_{i}\right)}{\left[1-p\left(1-q_{i}^{-a}\right)\right]^{3}}+\frac{p q_{i}^{-a} \ln \left(q_{i}\right)}{\left[1-p\left(1-q_{i}^{-a}\right)\right]^{2}}\right]\left[1-\frac{q_{i}^{-a}}{\left[1-p\left(1-q_{i}^{-a}\right)\right]}\right]^{-1}\right\} \\
& \boldsymbol{J}_{\sigma \beta_{k}}=\frac{a}{\sigma^{2}} \sum_{i \in F} \mathrm{v}_{i k}+\frac{(a+1)}{\sigma} \sum_{i \in F}\left[-\frac{\exp \left(\frac{y_{i}-\mathbf{v}_{i}^{T} \beta}{\sigma}\right) \mathrm{v}_{i k}}{\sigma\left[1+\exp \left(\frac{y_{i}-\mathbf{v}_{i}^{T} \beta}{\sigma}\right)\right]}-\frac{\left(\frac{y_{i}-\mathbf{v}_{i}^{T} \beta}{\sigma^{2}}\right) \exp \left(\frac{y_{i}-\mathbf{v}_{i}^{T} \beta}{\sigma}\right) \mathrm{v}_{i k}}{1+\exp \left(\frac{y_{i}-\mathbf{v}_{i}^{T} \beta}{\sigma}\right)}\right. \\
& \left.+\frac{\left(\frac{y_{i}-\mathbf{v}_{i}^{T} \beta}{\sigma^{2}}\right)\left[\exp \left(\frac{y_{i}-\mathbf{v}_{i}^{T} \beta}{\sigma}\right)\right]^{2} \mathrm{v}_{i k}}{\left[1+\exp \left(\frac{y_{i}-\mathbf{v}_{i}^{T} \beta}{\sigma}\right)\right]^{2}}\right]-\frac{2 p a}{\sigma^{2}} \sum_{i \in F} \frac{\exp \left[-\left(\frac{y_{i}-\mathbf{v}_{i}^{T} \beta}{\sigma}\right)\right] q_{i}^{-(a+1)} \mathrm{v}_{i k}}{1-p\left(1-q_{i}^{-a}\right)} \\
& +\frac{2 p a}{\sigma} \sum_{i \in F}\left[\frac{\left(\frac{y_{i}-\mathbf{v}_{i}^{T} \beta}{\sigma^{2}}\right) \exp \left[-\left(\frac{y_{i}-\mathbf{v}_{i}^{T} \beta}{\sigma}\right)\right] q_{i}^{-(a+1)} \mathrm{v}_{i k}}{1-p\left(1-q_{i}^{-a}\right)}\right.
\end{aligned}
$$




$$
\begin{aligned}
& +\frac{(-a-1)\left(\frac{y_{i}-\mathbf{v}_{i}^{T} \beta}{\sigma^{2}}\right)\left[\exp \left[-\left(\frac{y_{i}-\mathbf{v}_{i}^{T} \beta}{\sigma}\right)\right]\right]^{2} q_{i}^{-a-2} \mathbf{v}_{i k}}{1-p\left(1-q_{i}^{-a}\right)} \\
& \left.+\frac{p a\left(\frac{y_{i}-\mathbf{v}_{i}^{T} \beta}{\sigma^{2}}\right)\left[\exp \left[-\left(\frac{y_{i}-\mathbf{v}_{i}^{T} \beta}{\sigma}\right)\right]\right]^{2} q_{i}^{-a-2} \mathrm{v}_{i k}}{\left[1-p\left(1-q_{i}^{-a}\right)\right]^{2}}\right] \\
& +\sum_{i \in C}\left\{( \frac { y _ { i } - \mathbf { v } _ { i } ^ { T } \beta } { \sigma ^ { 2 } } ) \operatorname { e x p } [ - ( \frac { y _ { i } - \mathbf { v } _ { i } ^ { T } \beta } { \sigma } ) ] \mathbf { v } _ { i k } \left[\frac{p a q_{i}^{-2 a-1}}{\left[1-p\left(1-q_{i}^{-a}\right)\right]^{2}}\right.\right. \\
& \left.+\frac{1}{\left[1-p\left(1-q_{i}^{-a}\right)\right]}\right]\left[1-\frac{q_{i}^{-a}}{\left[1-p\left(1-q_{i}^{-a}\right)\right]}\right]^{-1} \\
& -\frac{\exp \left[-\left(\frac{y_{i}-\mathbf{v}_{i}^{T} \beta}{\sigma}\right)\right] \mathrm{v}_{i k}}{\sigma^{2}}\left[\frac{p a q_{i}^{-2 a-1}}{\left[1-p\left(1-q_{i}^{-a}\right)\right]^{2}}+\frac{1}{\left[1-p\left(1-q_{i}^{-a}\right)\right]}\right] \\
& \times\left[1-\frac{q_{i}^{-a}}{\left[1-p\left(1-q_{i}^{-a}\right)\right]}\right]^{-1}-\frac{1}{\sigma\left[1-q_{i}^{-a}\left[1-p\left(1-q_{i}^{-a}\right)\right]\right]^{2}} \\
& \times\left[\frac{p a \exp \left[-\left(\frac{y_{i}-\mathbf{v}_{i}^{T} \beta}{\sigma}\right)\right] \mathrm{v}_{i k} q_{i}^{-2 a-1}}{\left[1-p\left(1-q_{i}^{-a}\right)\right]^{2}}+\frac{\exp \left[-\left(\frac{y_{i}-\mathbf{v}_{i}^{T} \beta}{\sigma}\right)\right] \mathrm{v}_{i k}}{\left[1-p\left(1-q_{i}^{-a}\right)\right]}\right] \\
& \times\left[\frac{a\left(\frac{y_{i}-\mathbf{v}_{i}^{T} \beta}{\sigma^{2}}\right) \exp \left[-\left(\frac{y_{i}-\mathbf{v}_{i}^{T} \beta}{\sigma}\right)\right] q_{i}^{-a-1}}{\left[1-p\left(1-q_{i}^{-a}\right)\right]}-\frac{1}{\sigma\left[1-q_{i}^{-a}\left[1-p\left(1-q_{i}^{-a}\right)\right]\right]^{2}}\right] \\
& +\frac{1}{\sigma\left[1-q_{i}^{-a}\left[1-p\left(1-q_{i}^{-a}\right)\right]\right]}\left[\frac{p a(-2 a-1)\left(\frac{y_{i}-\mathbf{v}_{i}^{T} \beta}{\sigma^{2}}\right) \exp \left[-\left(\frac{y_{i}-\mathbf{v}_{i}^{T} \beta}{\sigma}\right)\right] q_{i}^{-2 a-2}}{\left[1-p\left(1-q_{i}^{-a}\right)\right]^{2}}\right. \\
& \left.\left.+\frac{2 p^{2} a^{2}\left(\frac{y_{i}-\mathbf{v}_{i}^{T} \beta}{\sigma^{2}}\right) \exp \left[-\left(\frac{y_{i}-\mathbf{v}_{i}^{T} \beta}{\sigma}\right)\right] q_{i}^{-3 a-2}}{\left[1-p\left(1-q_{i}^{-a}\right)\right]^{3}}+\frac{p a\left(\frac{y_{i}-\mathbf{v}_{i}^{T} \beta}{\sigma^{2}}\right) \exp \left[-\left(\frac{y_{i}-\mathbf{v}_{i}^{T} \beta}{\sigma}\right)\right] q_{i}^{-a-1}}{\left[1-p\left(1-q_{i}^{-a}\right)\right]^{2}}\right]\right\} \\
& \boldsymbol{J}_{p \beta_{k}}=\frac{2 a}{\sigma} \sum_{i \in F}\left[\frac{\exp \left[-\left(\frac{y_{i}-\mathbf{v}_{i}^{T} \beta}{\sigma}\right)\right] q_{i}^{-a-1} \mathrm{v}_{i k}}{\left[1-p\left(1-q_{i}^{-a}\right)\right]}-\frac{p \exp \left[-\left(\frac{y_{i}-\mathbf{v}_{i}^{T} \beta}{\sigma}\right)\right] q_{i}^{-a-1}\left(-1+q_{i}^{-a}\right)}{\left[1-p\left(1-q_{i}^{-a}\right)\right]^{2}}\right] \\
& +\sum_{i \in C}\left\{-\frac{\exp \left[-\left(\frac{y_{i}-\mathbf{v}_{i}^{T} \beta}{\sigma}\right)\right] q_{i}^{-a}\left(-1+q_{i}^{-a}\right) \mathrm{v}_{i k}}{\left[1-p\left(1-q_{i}^{-a}\right)\right]^{2}}\left[\frac{p a q_{i}^{-2 a-1}}{\left[1-p\left(1-q_{i}^{-a}\right)\right]^{2}}\right.\right. \\
& \left.+\frac{1}{\left[1-p\left(1-q_{i}^{-a}\right)\right]}\right]\left[1-\frac{q_{i}^{-a}}{\left[1-p\left(1-q_{i}^{-a}\right)\right]}\right]^{-2}+\exp \left[-\left(\frac{y_{i}-\mathbf{v}_{i}^{T} \beta}{\sigma}\right)\right] \mathrm{v}_{i k} \\
& \times\left[\frac{a q_{i}^{-2 a-1}}{\left[1-p\left(1-q_{i}^{-a}\right)\right]^{2}}-\frac{2 p a q_{i}^{-2 a-1}\left(-1+q_{i}^{-a}\right)}{\left[1-p\left(1-q_{i}^{-a}\right)\right]^{3}}-\frac{\left(-1+q_{i}^{-a}\right)}{\left[1-p\left(1-q_{i}^{-a}\right)\right]^{2}}\right] \\
& \left.\times\left[1-\frac{\left(-1+q_{i}^{-a}\right)}{\left[1-p\left(1-q_{i}^{-a}\right)\right]}\right]^{-1}\right\}
\end{aligned}
$$




$$
\begin{aligned}
\boldsymbol{J}_{\beta_{l} \beta_{k}}= & \frac{(a+1)}{\sigma^{2}} \sum_{i \in F}\left[-\frac{\exp \left(\frac{y_{i}-\mathbf{v}_{i}^{T} \beta}{\sigma}\right) \mathrm{v}_{i l} \mathrm{v}_{i k}}{\left[1+\exp \left(\frac{y_{i}-\mathbf{v}_{i}^{T} \beta}{\sigma}\right)\right]}+\frac{\left.\exp \left(\frac{y_{i}-\mathbf{v}_{i}^{T} \beta}{\sigma}\right)\right]^{2} \mathrm{v}_{i l} \mathrm{v}_{i k}}{\left[1+\exp \left(\frac{y_{i}-\mathbf{v}_{i}^{T} \beta}{\sigma}\right)\right]^{2}}\right] \\
& +\frac{2 p a}{\sigma} \sum_{i \in F}\left[\frac{\mathrm{H}^{\prime}\left(\exp \left[-\left(\frac{y_{i}-\mathbf{v}_{i}^{T} \beta}{\sigma}\right)\right] q_{i}^{-a-1} \mathrm{v}_{i k}\right)}{\left[1-p\left(1-q_{i}^{-a}\right)\right]}\right. \\
& \left.-\frac{\exp \left[-\left(\frac{y_{i}-\mathbf{v}_{i}^{T} \beta}{\sigma}\right)\right] q_{i}^{-a-1} \mathbf{v}_{i k} \mathrm{H}^{\prime}\left(1-p\left(1-q_{i}^{-a}\right)\right)}{\left[1-p\left(1-q_{i}^{-a}\right)\right]^{2}}\right] \\
& -\sum_{i \in C}\left\{\frac { 1 } { \sigma [ 1 - q _ { i } ^ { - a } [ 1 - p ( 1 - q _ { i } ^ { - a } ) ] ^ { - 1 } ] } \mathrm { H } ^ { \prime } \left[\frac{p a \exp \left[-\left(\frac{y_{i}-\mathbf{v}_{i}^{T} \beta}{\sigma}\right)\right] q_{i}^{-2 a-1} \mathrm{v}_{i k}}{\left[1-p\left(1-q_{i}^{-a}\right)\right]^{2}}\right.\right. \\
& \left.+\frac{\exp \left[-\left(\frac{y_{i}-\mathbf{v}_{i}^{T} \beta}{\sigma}\right)\right] \mathrm{v}_{i k}}{\left[1-p\left(1-q_{i}^{-a}\right)\right]}\right]+\left[\frac{p a \exp \left[-\left(\frac{y_{i}-\mathbf{v}_{i}^{T} \beta}{\sigma}\right)\right] q_{i}^{-2 a-1} \mathrm{v}_{i k}}{\left[1-p\left(1-q_{i}^{-a}\right)\right]^{2}}\right. \\
& \left.\left.+\frac{\exp \left[-\left(\frac{y_{i}-\mathbf{v}_{i}^{T} \beta}{\sigma}\right)\right] \mathrm{v}_{i k}}{\left[1-p\left(1-q_{i}^{-a}\right)\right]}\right] \mathrm{H}^{\prime}\left[\frac{1}{1-q_{i}^{-a}\left[1-p\left(1-q_{i}^{-a}\right)\right]^{-1}}\right]\right\}
\end{aligned}
$$




\section{Modelo LEGII}

$$
\begin{aligned}
& \boldsymbol{J}_{a a}=-\frac{r}{\alpha^{2}}+2 p \sum_{i \in F}\left[\frac{\log \left(q_{i}\right)^{2} q_{i}^{-a}}{1-p q_{i}^{-a}}+\frac{\log \left(q_{i}\right)^{2} q_{i}^{-2 a}}{\left[1-p q_{i}^{-a}\right]^{2}}\right]+\sum_{i \in C}\left\{\frac { 1 - p q _ { i } ^ { - a } } { 1 - q _ { i } ^ { - a } } \left[-\frac{(1-p) \log \left(q_{i}\right)^{2} q_{i}^{-a}}{1-p q_{i}^{-a}}\right.\right. \\
& \left.-\frac{3(1-p) p \log \left(q_{i}\right)^{2} q_{i}^{-2 a}}{\left[1-p q_{i}^{-a}\right]^{2}}-\frac{2(1-p) p^{2} \log \left(q_{i}\right)^{2} q_{i}^{-3 a}}{\left.\left[1-p q_{i}^{-a}\right)\right]^{3}}\right] \\
& \left.-\frac{\left[1-p q_{i}^{-a}\right]^{2}}{\left(1-q_{i}^{-a}\right)^{2}}\left[\frac{(1-p) \log \left(q_{i}\right) q_{i}^{-a}}{1-p q_{i}^{-a}}+\frac{(1-p) p \log \left(q_{i}\right) q_{i}^{-2 a}}{\left[1-p q_{i}^{-a}\right]^{2}}\right]^{2}\right\} \\
& \boldsymbol{J}_{a \sigma}=\sum_{i \in F}\left[-\left(\frac{y_{i}-\mathbf{v}_{i}^{T} \beta}{\sigma^{2}}\right)\right]+\sum_{i \in F} \frac{\left(\frac{y_{i}-\mathbf{v}_{i}^{T} \beta}{\sigma^{2}}\right) \exp \left(\frac{y_{i}-\mathbf{v}_{i}^{T} \beta}{\sigma}\right)}{1+\exp \left(\frac{y_{i}-\mathbf{v}_{i}^{T} \beta}{\sigma}\right)} \\
& -2 p \sum_{i \in F}\left[\frac{-a\left(\frac{y_{i}-\mathbf{v}_{i}^{T} \beta}{\sigma^{2}}\right) \exp \left[-\left(\frac{y_{i}-\mathbf{v}_{i}^{T} \beta}{\sigma}\right)\right] \log \left(q_{i}\right) q_{i}^{-(a+1)}}{1-p q_{i}^{-a}}\right. \\
& +\frac{\left(\frac{y_{i}-\mathbf{v}_{i}^{T} \beta}{\sigma^{2}}\right) \exp \left[-\left(\frac{y_{i}-\mathbf{v}_{i}^{T} \beta}{\sigma}\right)\right] q_{i}^{-(a+1)}}{1-p q_{i}^{-a}} \\
& \left.-\frac{\left.p a\left(\frac{y_{i}-\mathbf{v}_{i}^{T} \beta}{\sigma^{2}}\right) \exp \left[-\left(\frac{y_{i}-\mathbf{v}_{i}^{T} \beta}{\sigma}\right)\right] \log \left(q_{i}\right) q_{i}^{-(2 a+1)}\right]}{\left[1-p q_{i}^{-a}\right]^{2}}\right] \\
& +\sum_{i \in C}\left\{\frac { 1 - p q _ { i } ^ { - a } } { 1 - q _ { i } ^ { - a } } \left[-\frac{(1-p) a\left(\frac{y_{i}-\mathbf{v}_{i}^{T} \beta}{\sigma^{2}}\right) \exp \left[-\left(\frac{y_{i}-\mathbf{v}_{i}^{T} \beta}{\sigma}\right)\right] \log \left(q_{i}\right) q_{i}^{-(a+1)}}{1-p q_{i}^{-a}}\right.\right. \\
& +\frac{(1-p)\left(\frac{y_{i}-\mathbf{v}_{i}^{T} \beta}{\sigma^{2}}\right) \exp \left[-\left(\frac{y_{i}-\mathbf{v}_{i}^{T} \beta}{\sigma}\right)\right] q_{i}^{-(a+1)}}{1-p q_{i}^{-a}} \\
& -\frac{3(1-p) p a\left(\frac{y_{i}-\mathbf{v}_{i}^{T} \beta}{\sigma^{2}}\right) \exp \left[-\left(\frac{y_{i}-\mathbf{v}_{i}^{T} \beta}{\sigma}\right)\right] \log \left(q_{i}\right) q_{i}^{-(2 a+1)}}{\left[1-p q_{i}^{-a}\right]^{2}} \\
& -\frac{2(1-p) p^{2} a\left(\frac{y_{i}-\mathbf{v}_{i}^{T} \beta}{\sigma^{2}}\right) \exp \left[-\left(\frac{y_{i}-\mathbf{v}_{i}^{T} \beta}{\sigma}\right)\right] \log \left(q_{i}\right) q_{i}^{-(3 a+1)}}{\left[1-p q_{i}^{-a}\right]^{3}} \\
& \left.+\frac{(1-p) p\left(\frac{y_{i}-\mathbf{v}_{i}^{T} \beta}{\sigma^{2}}\right) \exp \left[-\left(\frac{y_{i}-\mathbf{v}_{i}^{T} \beta}{\sigma}\right)\right] q_{i}^{-(2 a+1)}}{\left[1-p q_{i}^{-a}\right]^{2}}\right] \\
& -\frac{\left[1-p q_{i}^{-a}\right]^{2}}{\left[1-q_{i}^{-a}\right]^{2}}\left[\frac{(1-p) a\left(\frac{y_{i}-\mathbf{v}_{i}^{T} \beta}{\sigma^{2}}\right) \exp \left[-\left(\frac{y_{i}-\mathbf{v}_{i}^{T} \beta}{\sigma}\right)\right] q_{i}^{-(a+1)}}{1-p q_{i}^{-a}}\right. \\
& \left.+\frac{(1-p) p a\left(\frac{y_{i}-\mathbf{v}_{i}^{T} \beta}{\sigma^{2}}\right) \exp \left[-\left(\frac{y_{i}-\mathbf{v}_{i}^{T} \beta}{\sigma}\right)\right] q_{i}^{-(2 a+1)}}{\left[1-p q_{i}^{-a}\right]^{2}}\right] \\
& \left.\times\left[\frac{(1-p) \log \left(q_{i}\right) q_{i}^{-a}}{1-p q_{i}^{-a}}+\frac{(1-p) p \log \left(q_{i}\right) q_{i}^{-2 a}}{\left[1-p q_{i}^{-a}\right]^{2}}\right]\right\}
\end{aligned}
$$




$$
\begin{aligned}
& \boldsymbol{J}_{a p}=-2 \sum_{i \in F}\left[\frac{\log \left(q_{i}\right) q_{i}^{-a}}{1-p q_{i}^{-a}}+\frac{p \log \left(q_{i}\right) q_{i}^{-2 a}}{1-p q_{i}^{-a}}\right]+\sum_{i \in C}\left\{\frac { 1 - p q _ { i } ^ { - a } } { 1 - q _ { i } ^ { - a } } \left[-\frac{\log \left(q_{i}\right) q_{i}^{-a}}{1-p q_{i}^{-a}}\right.\right. \\
& \left.-\frac{p \log \left(q_{i}\right) q_{i}^{-2 a}}{\left[1-p q_{i}^{-a}\right]^{2}}+\frac{2(1-p) \log \left(q_{i}\right) q_{i}^{-2 a}}{\left[1-p q_{i}^{-a}\right]^{2}}+\frac{2(1-p) \log \left(q_{i}\right) q_{i}^{-3 a}}{\left[1-p q_{i}^{-a}\right]^{3}}\right] \\
& -\frac{\left(1-p q_{i}^{-a}\right)^{2}}{\left(1-q_{i}^{-a}\right)^{2}}\left\{[ \frac { q _ { i } ^ { - a } } { 1 - p q _ { i } ^ { - a } } - \frac { ( 1 - p ) q _ { i } ^ { - 2 a } } { [ 1 - p q _ { i } ^ { - a } ] ^ { 2 } } ] \left[\frac{(1-p) \log \left(q_{i}\right) q_{i}^{-a}}{1-p q_{i}^{-a}}\right.\right. \\
& \left.\left.\left.+\frac{(1-p) p \log \left(q_{i}\right) q_{i}^{-2 a}}{\left[1-p q_{i}^{-a}\right]^{2}}\right]\right\}\right\} \\
& \boldsymbol{J}_{\sigma \sigma}=\frac{r}{\sigma^{2}}+2 a \sum_{i \in F}\left(\frac{y_{i}-\mathbf{v}_{i}^{T} \beta}{\sigma^{3}}\right)-(a+1) \sum_{i \in F}\left[\frac{2\left(\frac{y_{i}-\mathbf{v}_{i}^{T} \beta}{\sigma^{3}}\right) \exp \left(\frac{y_{i}-\mathbf{v}_{i}^{T} \beta}{\sigma}\right)}{1+\exp \left(\frac{y_{i}-\mathbf{v}_{i}^{T} \beta}{\sigma}\right)}\right. \\
& \left.+\frac{\left(\frac{y_{i}-\mathbf{v}_{i}^{T} \beta}{\sigma^{2}}\right)^{2} \exp \left(\frac{y_{i}-\mathbf{v}_{i}^{T} \beta}{\sigma}\right)}{1+\exp \left(\frac{y_{i}-\mathbf{v}_{i}^{T} \beta}{\sigma}\right)}-\frac{\left(\frac{y_{i}-\mathbf{v}_{i}^{T} \beta}{\sigma^{2}}\right)^{2}\left[\exp \left(\frac{y_{i}-\mathbf{v}_{i}^{T} \beta}{\sigma}\right)\right]^{2}}{\left[1+\exp \left(\frac{y_{i}-\mathbf{v}_{i}^{T} \beta}{\sigma}\right)\right]^{2}}\right]^{2} \\
& -2 p a \sum_{i \in F}\left[-\frac{a\left(\frac{y_{i}-\mathbf{v}_{i}^{T} \beta}{\sigma^{2}}\right)^{2}\left[\exp \left(-\frac{y_{i}-\mathbf{v}_{i}^{T} \beta}{\sigma}\right)\right]^{2} q_{i}^{-(a+2)}}{1-p q_{i}^{-a}}\right. \\
& -\frac{2\left(\frac{y_{i}-\mathbf{v}_{i}^{T} \beta}{\sigma^{3}}\right)\left[\exp \left(-\frac{y_{i}-\mathbf{v}_{i}^{T} \beta}{\sigma}\right)\right] q_{i}^{-(a+1)}}{1-p q_{i}^{-a}}+\frac{\left(\frac{y_{i}-\mathbf{v}_{i}^{T} \beta}{\sigma^{2}}\right)^{2}\left[\exp \left(-\frac{y_{i}-\mathbf{v}_{i}^{T} \beta}{\sigma}\right)\right] q_{i}^{-(a+1)}}{1-p q_{i}^{-a}} \\
& \left.-\frac{\left(\frac{y_{i}-\mathbf{v}_{i}^{T} \beta}{\sigma^{2}}\right)^{2}\left[\exp \left(-\frac{y_{i}-\mathbf{v}_{i}^{T} \beta}{\sigma}\right)\right]^{2} q_{i}^{-(a+2)}}{1-p q_{i}^{-a}}-\frac{p a\left(\frac{y_{i}-\mathbf{v}_{i}^{T} \beta}{\sigma^{2}}\right)^{2}\left[\exp \left(-\frac{y_{i}-\mathbf{v}_{i}^{T} \beta}{\sigma}\right)\right]^{2}}{\left[1-p q_{i}^{-a}\right]^{2}}\right] \\
& +\sum_{i \in C}\left\{\frac { 1 - p q _ { i } ^ { - a } } { 1 - q _ { i } ^ { - a } } \left[-\frac{(1-p) a^{2}\left(\frac{y_{i}-\mathbf{v}_{i}^{T} \beta}{\sigma^{2}}\right)^{2}\left[\exp \left(-\frac{y_{i}-\mathbf{v}_{i}^{T} \beta}{\sigma}\right)\right]^{2} q_{i}^{-(a+2)}}{1-p q_{i}^{-a}}\right.\right. \\
& -\frac{2(1-p) a\left(\frac{y_{i}-\mathbf{v}_{i}^{T} \beta}{\sigma^{3}}\right)\left[\exp \left(-\frac{y_{i}-\mathbf{v}_{i}^{T} \beta}{\sigma}\right)\right] q_{i}^{-(a+1)}}{1-p q_{i}^{-a}} \\
& +\frac{(1-p) a\left(\frac{y_{i}-\mathbf{v}_{i}^{T} \beta}{\sigma^{2}}\right)^{2}\left[\exp \left(-\frac{y_{i}-\mathbf{v}_{i}^{T} \beta}{\sigma}\right)\right] q_{i}^{-(a+1)}}{1-p q_{i}^{-a}} \\
& -\frac{(1-p) a\left(\frac{y_{i}-\mathbf{v}_{i}^{T} \beta}{\sigma^{2}}\right)^{2}\left[\exp \left(-\frac{y_{i}-\mathbf{v}_{i}^{T} \beta}{\sigma}\right)\right]^{2} q_{i}^{-(a+2)}}{1-p q_{i}^{-a}} \\
& -\frac{3(1-p) p a^{2}\left(\frac{y_{i}-\mathbf{v}_{i}^{T} \beta}{\sigma^{2}}\right)^{2}\left[\exp \left(-\frac{y_{i}-\mathbf{v}_{i}^{T} \beta}{\sigma}\right)\right]^{2} q_{i}^{-2(a+1)}}{\left[1-p q_{i}^{-a}\right]^{2}} \\
& -\frac{2(1-p) p^{2} a^{2}\left(\frac{y_{i}-\mathbf{v}_{i}^{T} \beta}{\sigma^{2}}\right)^{2}\left[\exp \left(-\frac{y_{i}-\mathbf{v}_{i}^{T} \beta}{\sigma}\right)\right]^{2} q_{i}^{-(3 a+2)}}{\left[1-p q_{i}^{-a}\right]^{3}} \\
& -\frac{2(1-p) p a\left(\frac{y_{i}-\mathbf{v}_{i}^{T} \beta}{\sigma^{3}}\right)\left[\exp \left(-\frac{y_{i}-\mathbf{v}_{i}^{T} \beta}{\sigma}\right)\right] q_{i}^{-(2 a+1)}}{\left[1-p q_{i}^{-a}\right]^{2}}
\end{aligned}
$$




$$
\begin{aligned}
& +\frac{(1-p) p a\left(\frac{y_{i}-\mathbf{v}_{i}^{T} \beta}{\sigma^{2}}\right)^{2}\left[\exp \left(-\frac{y_{i}-\mathbf{v}_{i}^{T} \beta}{\sigma}\right)\right] q_{i}^{-(2 a+1)}}{\left[1-p q_{i}^{-a}\right]^{2}} \\
& \left.-\frac{(1-p) p a\left(\frac{y_{i}-\mathbf{v}_{i}^{T} \beta}{\sigma^{2}}\right)^{2}\left[\exp \left(-\frac{y_{i}-\mathbf{v}_{i}^{T} \beta}{\sigma}\right)\right]^{2} q_{i}^{-2(a+1)}}{\left[1-p q_{i}^{-a}\right]^{2}}\right] \\
& -\frac{\left[1-p q_{i}^{-a}\right]^{2}}{\left[1-q_{i}^{-a}\right]^{2}}\left[\frac{(1-p) a\left(\frac{y_{i}-\mathbf{v}_{i}^{T} \beta}{\sigma^{2}}\right)\left[\exp \left(-\frac{y_{i}-\mathbf{v}_{i}^{T} \beta}{\sigma}\right)\right] q_{i}^{-(a+1)}}{1-p q_{i}^{-a}}\right. \\
& \left.\left.+\frac{(1-p) p a\left(\frac{y_{i}-\mathbf{v}_{i}^{T} \beta}{\sigma^{2}}\right)\left[\exp \left(-\frac{y_{i}-\mathbf{v}_{i}^{T} \beta}{\sigma}\right)\right] q_{i}^{-(2 a+1)}}{\left[1-p q_{i}^{-a}\right]^{2}}\right]^{2}\right\} \\
& \boldsymbol{J}_{\sigma p}=-2 a \sum_{i \in F}\left[\frac{\left(\frac{y_{i}-\mathbf{v}_{i}^{T} \beta}{\sigma^{2}}\right)\left[\exp \left(-\frac{y_{i}-\mathbf{v}_{i}^{T} \beta}{\sigma}\right)\right] q_{i}^{-(a+1)}}{1-p q_{i}^{-a}}+\frac{p\left(\frac{y_{i}-\mathbf{v}_{i}^{T} \beta}{\sigma^{2}}\right) \exp \left(-\frac{y_{i}-\mathbf{v}_{i}^{T} \beta}{\sigma}\right) q_{i}^{-(2 a+1)}}{\left[1-p q_{i}^{-a}\right]^{2}}\right] \\
& +\sum_{i \in C}\left\{\frac { 1 - p q _ { i } ^ { - a } } { 1 - q _ { i } ^ { - a } } \left[-\frac{a\left(\frac{y_{i}-\mathbf{v}_{i}^{T} \beta}{\sigma^{2}}\right)\left[\exp \left(-\frac{y_{i}-\mathbf{v}_{i}^{T} \beta}{\sigma}\right)\right] q_{i}^{-(a+1)}}{1-p q_{i}^{-a}}\right.\right. \\
& -\frac{p a\left(\frac{y_{i}-\mathbf{v}_{i}^{T} \beta}{\sigma^{2}}\right)\left[\exp \left(-\frac{y_{i}-\mathbf{v}_{i}^{T} \beta}{\sigma}\right)\right] q_{i}^{-(2 a+1)}}{\left[1-p q_{i}^{-a}\right]^{2}} \\
& +\frac{2(1-p) a\left(\frac{y_{i}-\mathbf{v}_{i}^{T} \beta}{\sigma^{2}}\right)\left[\exp \left(-\frac{y_{i}-\mathbf{v}_{i}^{T} \beta}{\sigma}\right)\right] q_{i}^{-(2 a+1)}}{\left[1-p q_{i}^{-a}\right]^{2}} \\
& \left.+\frac{2(1-p) p a\left(\frac{y_{i}-\mathbf{v}_{i}^{T} \beta}{\sigma^{2}}\right)\left[\exp \left(-\frac{y_{i}-\mathbf{v}_{i}^{T} \beta}{\sigma}\right)\right] q_{i}^{-(3 a+1)}}{\left[1-p q_{i}^{-a}\right]^{3}}\right]-\frac{\left(1-p q_{i}^{-a}\right)^{2}}{\left(1-q_{i}^{-a}\right)^{2}}\left\{\left[\frac{q_{i}^{-a}}{1-p q_{i}^{-a}}\right.\right. \\
& \left.-\frac{(1-p) q_{i}^{-2 a}}{\left[1-p q_{i}^{-a}\right]^{2}}\right]\left[\frac{(1-p) a\left(\frac{y_{i}-\mathbf{v}_{i}^{T} \beta}{\sigma^{2}}\right)\left[\exp \left(-\frac{y_{i}-\mathbf{v}_{i}^{T} \beta}{\sigma}\right)\right] q_{i}^{-(a+1)}}{1-p q_{i}^{-a}}\right. \\
& \left.\left.\left.+\frac{(1-p) p a\left(\frac{y_{i}-\mathbf{v}_{i}^{T} \beta}{\sigma^{2}}\right)\left[\exp \left(-\frac{y_{i}-\mathbf{v}_{i}^{T} \beta}{\sigma}\right)\right] q_{i}^{-(2 a+1)}}{\left[1-p q_{i}^{-a}\right]^{2}}\right]\right\}\right\} \\
& \boldsymbol{J}_{p p}=-\frac{r}{(1-p)^{2}}+2 \sum_{i \in F} \frac{q_{i}^{-2 a}}{\left[1-p q_{i}^{-a}\right]^{2}}+\sum_{i \in C}\left\{\frac{1-p q_{i}^{-a}}{1-q_{i}^{-a}}\left[\frac{2 q_{i}^{-2 a}}{\left[1-p q_{i}^{-a}\right]^{2}}-\frac{2(1-p) q_{i}^{-3 a}}{\left[1-p q_{i}^{-a}\right]^{3}}\right]\right. \\
& \left.-\frac{\left(1-p q_{i}^{-a}\right)^{2}}{\left(1-q_{i}^{-a}\right)^{2}}\left[\frac{q_{i}^{-a}}{1-p q_{i}^{-a}}-\frac{(1-p) q_{i}^{-2 a}}{\left[1-p q_{i}^{-a}\right]^{2}}\right]^{2}\right\} \\
& \boldsymbol{J}_{a \beta_{k}}=-\frac{1}{\sigma} \sum_{i \in F} \mathrm{v}_{i k}+\frac{1}{\sigma} \sum_{i \in F} \frac{\exp \left(\frac{y_{i}-\mathbf{v}_{i}^{T} \beta}{\sigma}\right) \mathrm{v}_{i k}}{1+\exp \left(\frac{y_{i}-\mathbf{v}_{i}^{T} \beta}{\sigma}\right)}-\frac{2 p}{\sigma} \sum_{i \in F}\left\{\frac{\exp \left[-\left(\frac{y_{i}-\mathbf{v}_{i}^{T} \beta}{\sigma}\right)\right] q_{i}^{-(a+1)} \mathrm{v}_{i k}}{1-p q_{i}^{-a}}\right. \\
& \left.-\frac{a \exp \left[-\left(\frac{y_{i}-\mathbf{v}_{i}^{T} \beta}{\sigma}\right)\right] q_{i}^{-(a+1)} \ln \left(q_{i}\right) \mathrm{v}_{i k}}{\left[1-p q_{i}^{-a}\right]}-\frac{p a \exp \left[-\left(\frac{y_{i}-\mathbf{v}_{i}^{T} \beta}{\sigma}\right)\right] q_{i}^{-2 a-1} \ln \left(q_{i}\right) \mathrm{v}_{i k}}{\left[1-p q_{i}^{-a}\right]^{2}}\right\}
\end{aligned}
$$




$$
\begin{aligned}
& -\frac{1}{\sigma} \sum_{i \in C}\left\{-\exp \left[-\left(\frac{y_{i}-\mathbf{v}_{i}^{T} \beta}{\sigma}\right)\right] \mathbf{v}_{i k}\left[-\frac{(1-p) p a q_{i}^{-2 a-1}}{\left[1-p q_{i}^{-a}\right]^{2}}+\frac{(1-p)}{1-p q_{i}^{-a}}\right]\right. \\
& \times\left[\frac{(1-p) q_{i}^{-a} \ln \left(q_{i}\right)}{1-p q_{i}^{-a}}+\frac{(1-p) p q_{i}^{-2 a} \ln \left(q_{i}\right)}{\left[1-p q_{i}^{-a}\right]^{2}}\right]\left[1-\frac{(1-p) q_{i}^{-a}}{1-p q_{i}^{-a}}\right]^{-2} \\
& +\exp \left[-\left(\frac{y_{i}-\mathbf{v}_{i}^{T} \beta}{\sigma}\right)\right] \mathrm{v}_{i k}\left[-\frac{(1-p) p q_{i}^{-2 a-1}}{\left[1-p q_{i}^{-a}\right]^{2}}+\frac{2(1-p) p a q_{i}^{-2 a-1} \ln \left(q_{i}\right)}{\left[1-p q_{i}^{-a}\right]^{2}}\right. \\
& \left.\left.+\frac{2(1-p) p^{2} a q_{i}^{-3 a-1} \ln \left(q_{i}\right)}{\left[1-p q_{i}^{-a}\right]^{3}}-\frac{(1-p) p q_{i}^{-a} \ln \left(q_{i}\right)}{\left[1-p q_{i}^{-a}\right]^{2}}\right]\left[1-\frac{(1-p) q_{i}^{-a}}{\left[1-p q_{i}^{-a}\right]}\right]^{-1}\right\} \\
& \boldsymbol{J}_{\sigma \beta_{k}}=\frac{a}{\sigma^{2}} \sum_{i \in F} \mathrm{v}_{i k}+\frac{(a+1)}{\sigma} \sum_{i \in F}\left[-\frac{\exp \left(\frac{y_{i}-\mathbf{v}_{i}^{T} \beta}{\sigma}\right) \mathrm{v}_{i k}}{\sigma\left[1+\exp \left(\frac{y_{i}-\mathbf{v}_{i}^{T} \beta}{\sigma}\right)\right]}-\frac{\left(\frac{y_{i}-\mathbf{v}_{i}^{T} \beta}{\sigma^{2}}\right) \exp \left(\frac{y_{i}-\mathbf{v}_{i}^{T} \beta}{\sigma}\right) \mathrm{v}_{i k}}{1+\exp \left(\frac{y_{i}-\mathbf{v}_{i}^{T} \beta}{\sigma}\right)}\right. \\
& \left.+\frac{\left(\frac{y_{i}-\mathbf{v}_{i}^{T} \beta}{\sigma^{2}}\right)\left[\exp \left(\frac{y_{i}-\mathbf{v}_{i}^{T} \beta}{\sigma}\right)\right]^{2} \mathrm{v}_{i k}}{\left[1+\exp \left(\frac{y_{i}-\mathbf{v}_{i}^{T} \beta}{\sigma}\right)\right]^{2}}\right]-\frac{2 p a}{\sigma^{2}} \sum_{i \in F} \frac{\exp \left[-\left(\frac{y_{i}-\mathbf{v}_{i}^{T} \beta}{\sigma}\right)\right] q_{i}^{-(a+1)} \mathrm{v}_{i k}}{1-p q_{i}^{-a}} \\
& +\frac{2 p a}{\sigma} \sum_{i \in F}\left[\frac{\left(\frac{y_{i}-\mathbf{v}_{i}^{T} \beta}{\sigma^{2}}\right) \exp \left[-\left(\frac{y_{i}-\mathbf{v}_{i}^{T} \beta}{\sigma}\right)\right] q_{i}^{-(a+1)} \mathrm{v}_{i k}}{1-p q_{i}^{-a}}\right. \\
& +\frac{(-a-1)\left(\frac{y_{i}-\mathbf{v}_{i}^{T} \beta}{\sigma^{2}}\right)\left[\exp \left[-\left(\frac{y_{i}-\mathbf{v}_{i}^{T} \beta}{\sigma}\right)\right]\right]^{2} q_{i}^{-a-2} \mathrm{v}_{i k}}{1-p q_{i}^{-a}} \\
& \left.-\frac{p a\left(\frac{y_{i}-\mathbf{v}_{i}^{T} \beta}{\sigma^{2}}\right)\left[\exp \left[-\left(\frac{y_{i}-\mathbf{v}_{i}^{T} \beta}{\sigma}\right)\right]\right]^{2} q_{i}^{-2 a-2} \mathrm{v}_{i k}}{\left[1-p q_{i}^{-a}\right]^{2}}\right] \\
& -\sum_{i \in C}\left\{( \frac { y _ { i } - \mathbf { v } _ { i } ^ { T } \beta } { \sigma ^ { 2 } } ) \operatorname { e x p } [ - ( \frac { y _ { i } - \mathbf { v } _ { i } ^ { T } \beta } { \sigma } ) ] \mathbf { v } _ { i k } \left[-\frac{(1-p) p a q_{i}^{-2 a-1}}{\left[1-p q_{i}^{-a}\right]^{2}}\right.\right. \\
& \left.+\frac{(1-p)}{\left[1-p q_{i}^{-a}\right]}\right]\left[1-\frac{(1-p) q_{i}^{-a}}{\left[1-p q_{i}^{-a}\right]}\right]^{-1}-\frac{\exp \left[-\left(\frac{y_{i}-\mathbf{v}_{i}^{T} \beta}{\sigma}\right)\right] \mathrm{v}_{i k}}{\sigma^{2}} \\
& \times\left[-\frac{(1-p) p a q_{i}^{-2 a-1}}{\left[1-p q_{i}^{-a}\right]^{2}}+\frac{(1-p)}{\left[1-p q_{i}^{-a}\right]}\right]\left[1-\frac{(1-p) q_{i}^{-a}}{\left[1-p q_{i}^{-a}\right]}\right]^{-1} \\
& -\left[-\frac{(1-p) p a \exp \left[-\left(\frac{y_{i}-\mathbf{v}_{i}^{T} \beta}{\sigma}\right)\right] \mathrm{v}_{i k} q_{i}^{-2 a-1}}{\sigma\left[1-p q_{i}^{-a}\right]^{2}}+\frac{(1-p) \exp \left[-\left(\frac{y_{i}-\mathbf{v}_{i}^{T} \beta}{\sigma}\right)\right] \mathrm{v}_{i k}}{\sigma\left[1-p q_{i}^{-a}\right]}\right] \\
& \times\left[\frac{(1-p) a\left(\frac{y_{i}-\mathbf{v}_{i}^{T} \beta}{\sigma^{2}}\right) \exp \left[-\left(\frac{y_{i}-\mathbf{v}_{i}^{T} \beta}{\sigma}\right)\right] q_{i}^{-a-1}}{\left[1-p q_{i}^{-a}\right]}\right.
\end{aligned}
$$




$$
\begin{aligned}
& \left.+\frac{(1-p) p a\left(\frac{y_{i}-\mathbf{v}_{i}^{T} \beta}{\sigma^{2}}\right) \exp \left[-\left(\frac{y_{i}-\mathbf{v}_{i}^{T} \beta}{\sigma}\right)\right] q_{i}^{-2 a-1}}{\left[1-p q_{i}^{-a}\right]^{2}}\right]\left[1-\frac{(1-p) q_{i}^{-a}}{\left[1-p q_{i}^{-a}\right]}\right]^{-2} \\
& +\exp \left[-\left(\frac{y_{i}-\mathbf{v}_{i}^{T} \beta}{\sigma}\right)\right] \mathbf{v}_{i k}\left[\frac{(1-p) p a(-2 a-1)\left(\frac{y_{i}-\mathbf{v}_{i}^{T} \beta}{\sigma^{2}}\right) \exp \left[-\left(\frac{y_{i}-\mathbf{v}_{i}^{T} \beta}{\sigma}\right)\right] q_{i}^{-2 a-1}}{\left[1-p q_{i}^{-a}\right]^{2}}\right. \\
& +\frac{2(1-p) p^{2} a^{2}\left(\frac{y_{i}-\mathbf{v}_{i}^{T} \beta}{\sigma^{2}}\right) \exp \left[-\left(\frac{y_{i}-\mathbf{v}_{i}^{T} \beta}{\sigma}\right)\right] q_{i}^{-3 a-2}}{\left[1-p q_{i}^{-a}\right]^{3}} \\
& \left.\left.-\frac{(1-p) p a\left(\frac{y_{i}-\mathbf{v}_{i}^{T} \beta}{\sigma^{2}}\right) \exp \left[-\left(\frac{y_{i}-\mathbf{v}_{i}^{T} \beta}{\sigma}\right)\right] q_{i}^{-a-1}}{\left[1-p q_{i}^{-a}\right]^{2}}\right]\left[1-\frac{(1-p) q_{i}^{-a}}{\left[1-p q_{i}^{-a}\right]}\right]^{-1}\right\} \\
& \boldsymbol{J}_{p \beta_{k}}=\frac{2 a}{\sigma} \sum_{i \in F}\left[\frac{\exp \left[-\left(\frac{y_{i}-\mathbf{v}_{i}^{T} \beta}{\sigma}\right)\right] q_{i}^{-a-1} \mathrm{v}_{i k}}{\left[1-p q_{i}^{-a}\right]}+\frac{p \exp \left[-\left(\frac{y_{i}-\mathbf{v}_{i}^{T} \beta}{\sigma}\right)\right] q_{i}^{-2 a-1}}{\left[1-p q_{i}^{-a}\right]^{2}}\right] \\
& -\frac{1}{\sigma} \sum_{i \in C}\left\{-\exp \left[-\left(\frac{y_{i}-\mathbf{v}_{i}^{T} \beta}{\sigma}\right)\right] \mathbf{v}_{i k}\left[-\frac{(1-p) p a q_{i}^{-2 a-1}}{\left[1-p q_{i}^{-a}\right]^{2}}\right.\right. \\
& \left.+\frac{(1-p)}{\left[1-p q_{i}^{-a}\right]}\right]\left[\frac{q_{i}^{-a}}{\left[1-p q_{i}^{-a}\right]}-\frac{(1-p) q_{i}^{-2 a}}{\left[1-p q_{i}^{-a}\right]^{2}}\right]\left[1-\frac{(1-p) q_{i}^{-a}}{\left[1-p q_{i}^{-a}\right]}\right]^{-2} \\
& +\exp \left[-\left(\frac{y_{i}-\mathbf{v}_{i}^{T} \beta}{\sigma}\right)\right] \mathbf{v}_{i k}\left[\frac{p a q_{i}^{-2 a-1}}{\left[1-p q_{i}^{-a}\right]^{2}}-\frac{(1-p) a q_{i}^{-2 a-1}}{\left[1-p q_{i}^{-a}\right]^{2}}-\right. \\
& \left.\left.\frac{2(1-p) p a q_{i}^{-3 a-1}}{\left[1-p q_{i}^{-a}\right]^{3}}-\frac{1}{\left[1-p q_{i}^{-a}\right]}+\frac{(1-p) q_{i}^{-a}}{\left[1-p q_{i}^{-a}\right]^{2}}\right]\left[1-\frac{(1-p) q_{i}^{-a}}{\left[1-p q_{i}^{-a}\right]}\right]^{-1}\right\} \\
& \boldsymbol{J}_{\beta_{l} \beta_{k}}=\frac{(a+1)}{\sigma^{2}} \sum_{i \in F}\left[-\frac{\exp \left(\frac{y_{i}-\mathbf{v}_{i}^{T} \beta}{\sigma}\right) \mathrm{v}_{i l} \mathrm{v}_{i k}}{\left[1+\exp \left(\frac{y_{i}-\mathbf{v}_{i}^{T} \beta}{\sigma}\right)\right]}+\frac{\left[\exp \left(\frac{y_{i}-\mathbf{v}_{i}^{T} \beta}{\sigma}\right)\right]^{2} \mathrm{v}_{i l} \mathbf{v}_{i k}}{\left[1+\exp \left(\frac{y_{i}-\mathbf{v}_{i}^{T} \beta}{\sigma}\right)\right]^{2}}\right]- \\
& \frac{2 p a}{\sigma} \sum_{i \in F}\left[\frac{\mathrm{H}^{\prime}\left(\exp \left[-\left(\frac{y_{i}-\mathbf{v}_{i}^{T} \beta}{\sigma}\right)\right] q_{i}^{-a-1} \mathrm{v}_{i k}\right)}{\left[1-p q_{i}^{-a}\right]}-\right. \\
& \left.\frac{\exp \left[-\left(\frac{y_{i}-\mathbf{v}_{i}^{T} \beta}{\sigma}\right)\right] q_{i}^{-a-1} \mathrm{v}_{i k} \mathrm{H}^{\prime}\left(1-p q_{i}^{-a}\right)}{\left[1-p q_{i}^{-a}\right]^{2}}\right]- \\
& \sum_{i \in C}\left\{\frac { 1 } { \sigma [ 1 - ( 1 - p ) q _ { i } ^ { - a } [ 1 - p q _ { i } ^ { - a } ] ^ { - 1 } ] } \mathrm { H } ^ { \prime } \left[-\frac{(1-p) p a \exp \left[-\left(\frac{y_{i}-\mathbf{v}_{i}^{T} \beta}{\sigma}\right)\right] q_{i}^{-2 a-1} \mathrm{v}_{i k}}{\sigma\left[1-p q_{i}^{-a}\right]^{2}}+\right.\right. \\
& \left.\frac{(1-p) \exp \left[-\left(\frac{y_{i}-\mathbf{v}_{i}^{T} \beta}{\sigma}\right)\right] \mathrm{v}_{i k}}{\sigma\left[1-p q_{i}^{-a}\right]}\right]+\left[-\frac{(1-p) p a \exp \left[-\left(\frac{y_{i}-\mathbf{v}_{i}^{T} \beta}{\sigma}\right)\right] q_{i}^{-2 a-1} \mathrm{v}_{i k}}{\sigma\left[1-p q_{i}^{-a}\right]^{2}}+\right. \\
& \left.\left.\frac{(1-p) \exp \left[-\left(\frac{y_{i}-\mathbf{v}_{i}^{T} \beta}{\sigma}\right)\right] \mathrm{v}_{i k}}{\left[1-p q_{i}^{-a}\right]}\right] \mathrm{H}^{\prime}\left[\frac{1}{1-(1-p) q_{i}^{-a}\left[1-p q_{i}^{-a}\right]^{-1}}\right]\right\}
\end{aligned}
$$

em que $\mathrm{H}^{\prime}($.$) é função derivável em relação a \beta_{l}$, para $k, l=1, \ldots, q$. 